\title{
Reactions of Aqueous
}

Aluminum Species

at Mineral Surfaces

GEOLOGICAL SURVEY WATER-SUPPLY PAPER 1827-F

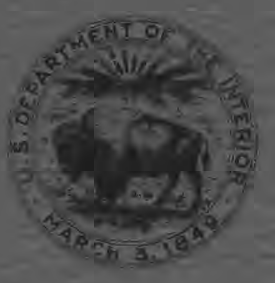




\section{Reactions of Aqueous}

Aluminum Species

at Mineral Surfaces

By D. W. BROWN and J. D. HEM

CHEMISTRY OF ALUMINUM IN NATURAL WATER

GEOLOGICAL SURVEY WATER-SUPPLY PAPER 1827-F

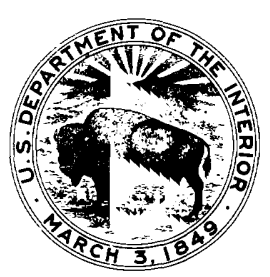

UNITED STATES GOVERNMENT PRINTING OFFICE, WASHINGTON : 1975 


\title{
UNITED STATES DEPARTMENT OF THE INTERICR
}

ROGERS C. B. MORTON, Secretary

\author{
GEOLOGICAL SURVEY
}

V. E. McKelvey, Director

Library of Congress Cataloging in Publication Data

Brown, David Wayne, 1949-

Reactions of aqueous aluminum species at mineral surfaces. (Chemistry of aluminum in natural water)

(Geological Survey Water-supply Paper 1827-F

Bibliography: p. 48.

1. Aluminum salts. 2. Surface chemistry. 3. Water chemistry. I. Hem, John David, 1916- joint author. II. Title. III. Series. IV. Series: United States. Geological Survey. Water-supply Paper 1827-F.

TC801.U2 [QD181.A4] 553'.7'0973s [551.4'9] 75-619064

For sale by the Superintendent of Documents, U. S. Government Printing Office Washington, D. C. 20402

Stock No. 024-001-02657-1 


\section{CONTENTS}

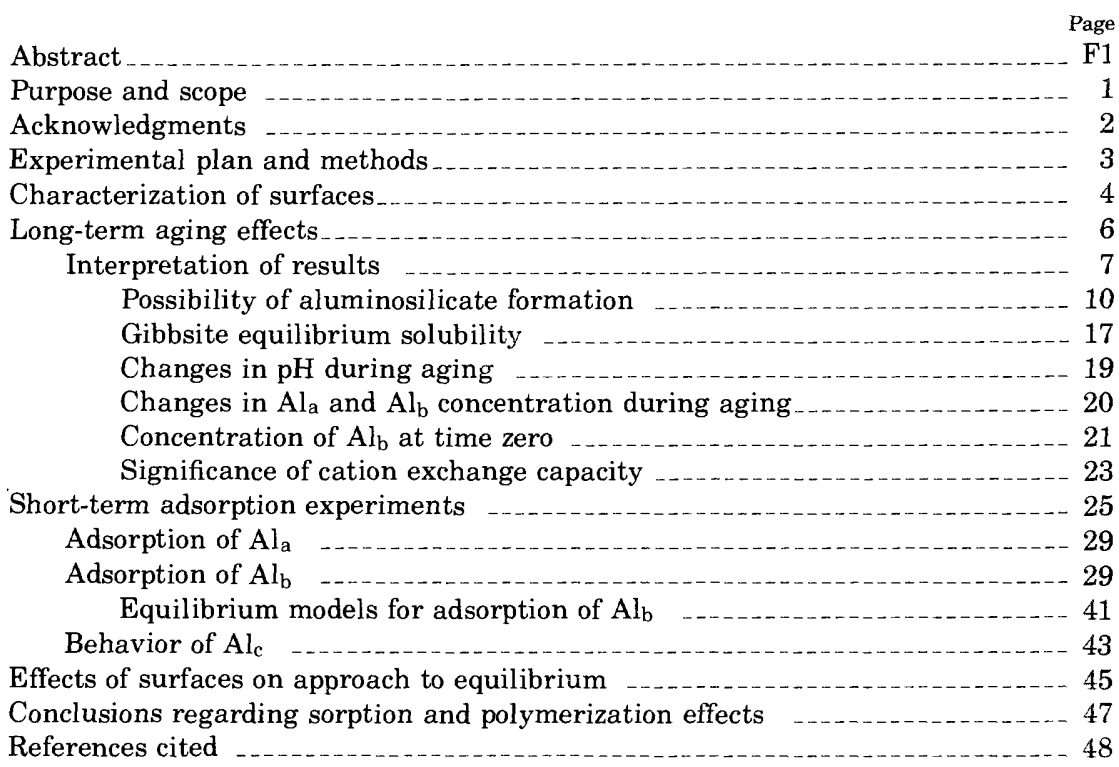

\section{ILLUSTRATIONS}

Figure 1. Graph showing relation between specific surface area and cation exchange capacity for materials studied _.............. F7

2. Electron micrograph of Wyoming bentonite

3. Electron micrograph of volcanic ash

4. Electron micrograph of kaolinite

5. Diagram comparing compositions of experimental solutions with

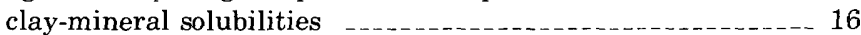

6. Diagram comparing compositions of experimental solutions with gibbsite solubilities _..._._. 18

7. Graph showing first-order disappearance of $\mathrm{Al}_{\mathrm{b}}$ and $\mathrm{pH}$ chang $9 \mathrm{~s}$ for

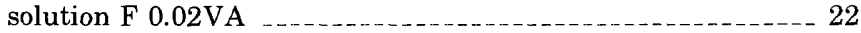

8. Graph showing first-order disappearance of $\mathrm{Al}_{\mathrm{b}}$ and $\mathrm{pH}$ chang $9 \mathrm{~s}$ for solution $\mathrm{H} 0.1 \mathrm{~K} \ldots \ldots \ldots$

9-12. Graphs showing initial loss of $\mathrm{Al}_{\mathrm{b}}$ as a function of surface area in:

9. Solutions D .... 25

10. Solutions F _. 26 
FIgures 9-12. Graphs showing initial loss of $\mathrm{Al}_{\mathrm{b}}$ as a function of surface area Page in-Continued

11. Solutions G

12. Solutions H

13-18. Graphs showing:

13. Adsorption of $\mathrm{Al}_{\mathrm{a}}$ at 1 and 48 hours in soluticns $\mathrm{DS}$ as a

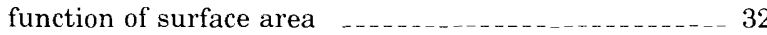

14. Gibbsite ion activity product in solutions DS as a function of cation exchange capacity _._. 36

15. Adsorption of $\mathrm{Al}_{\mathrm{b}}$ at 1 and 48 hours in soluticns DS as a function of surface area

16. Adsorption of $\mathrm{Al}_{\mathrm{b}}$ at 1 and 48 hours in soluticns DS as a function of cation exchange capacity _._._._._. 39

17. Adsorption of $\mathrm{Al}_{\mathrm{b}}$ as a function of time of solution-surface contact - _.

18. Test of $\mathrm{Al}_{\mathrm{b}}$ adsorption at 48 hours for corresfondence to Langmuir Isotherm

19-22. Electron micrographs of:

19. Adsorbed microcrystalline gibbsite on Wyoming bentonite 44

20. Adsorbed microcrystalline gibbsite on volcanic ash $\ldots . . . . .45$

21. Adsorbed microcrystalline gibbsite on kaolinite _......- 46

22. Microcrystalline gibbsite adhering to kaolinite after 48

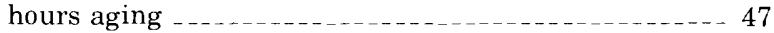

\section{TABLES}

TABLES 1-4. Concentrations of two types of dissolved aluminum and $\mathrm{pH}$ in relation to age and amount and type of mineral surface in:

1. Solutions D _ F11

2. Solutions F .

3. Solutions G

4. Solutions $\mathrm{H}$

5. Aluminosilicate and gibbsite ion activity products and related data for solutions $\mathrm{D}, \mathrm{F}, \mathrm{G}$, and $\mathrm{H}$ after 2-3 months aging 15

6. Short-term losses of $\mathrm{Al}_{\mathrm{b}}$ in solutions $\mathrm{D}, \mathrm{F}, \mathrm{G}$, and $\mathrm{H}_{\ldots} \ldots$

7. Adsorption experiment data _._.

8. Calculated sorbed species and aqueous $\mathrm{Al}_{\mathrm{c}}$ concentrations _..... 30

9. Calculated aqueous $\mathrm{Al}_{\mathrm{a}}$ species distribution at 48 hours _..._. 31

10. Dissolved and sorbed species and selectivity constants for three ion exchange mechanisms _.................... 34

11. Data used for development of Langmuir isotherm for ac'sorption of $\mathrm{Al}_{\mathrm{b}} \ldots \ldots$ 


\title{
REACTIONS OF AQUEOUS ALUMINUM SPECIES AT MINERAL SURFACES
}

\author{
By D. W. Brown and J. D. HEM
}

\section{ABSTRACT}

Aqueous aluminum solutions containing $4.5 \times 10^{-4}$ molar aluminum in 0.01 molar $\mathrm{NaClO}_{4}$ were partly neutralized with $\mathrm{NaOH}$ to give $\mathrm{OH}$ : Al mole ratios from 1.40 to 2.76. Measured amounts of montmorillonite, kaolinite, volcanic ash, or feldspathic sand were added to provide an area of inert surface. Reactions that occurred during 100 days of aging were compared with those in similar solutions without added surfaces, studied in earlier work.

Adsorption of monomeric species $\mathrm{Al}\left(\mathrm{H}_{2} \mathrm{O}\right)_{6}{ }^{+3}, \mathrm{AlOH}\left(\mathrm{H}_{2} \mathrm{O}\right)_{5}{ }^{+2}$, and $\mathrm{Al}(\mathrm{OH})_{2}\left(\mathrm{H}_{2} \mathrm{O}\right)_{4}{ }^{+}$on the added surfaces follows a cation exchange mass law equilibrium model, and adsorption is essentially complete in 1 hour. Only minor changes in monomeric aluminum species occurred after that.

Rapid adsorption of polynuclear aluminum hydroxide species also occurs ard follows the pattern of the Langmuir adsorption isotherm. In the absence of surfaces, the polynuclear ions slowly increase in size and become microcrystalline gibbsite during aging. Electron micrographs showed microcrystalline gibbsite was present or surfaces after aging only 2 days. However, the analytical data suggest this material must have been adsorbed after it had already attained a near-crystalline state. Adsorbed polynuclear aluminum hydroxide species were not extensively converted to microcrystalline gibbsite during 100 days of aging.

\section{PURPOSE AND SCOPE}

In an earlier paper in this series, Hem and Roberson (1967) dealt with the form and stability of aqueous alumin um hydroxide complexes at various ranges of $\mathrm{pH}$. They reported that below neutral $\mathrm{pH}$ the dissolved aluminum species consist of three forms, (1) the monomeric octahedral $\mathrm{Al}\left(\mathrm{H}_{2} \mathrm{O}\right)_{6}+3, \quad \mathrm{AlOH}\left(\mathrm{H}_{2} \mathrm{O}\right)_{5}+2, \quad \mathrm{Al}(\mathrm{OH})_{2}\left(\mathrm{H}_{2} \mathrm{O}\right)_{4}{ }^{+}$, and $\mathrm{Al}(\mathrm{OH})_{4}\left(\mathrm{H}_{2} \mathrm{O}_{2}{ }^{-}\right.$ions, (2) a positively charged polymeric species consisting of octahedral units held tugether by double hydroxide bridges and containing as much as several hundred aluminum atoms, and (3) hexagonal microcrystalline gibbsite particles with maximum diameters near $0.1 \mu \mathrm{m}$ (micrometre). In a later report, Smith and Her (1972) described the effect of aging on these species designated by them $\mathrm{Al}_{\mathrm{a}}$, $\mathrm{Al}_{\mathrm{b}}$, and $\mathrm{Al}_{\boldsymbol{c}}$, respectively. No clearly defined boundary exists botween 
$\mathrm{Al}_{\mathrm{b}}$ and $\mathrm{Al}_{\mathrm{c}}$ with regard to molecular size; the boundary used for convenience is an analytical one. When $\mathrm{Al}_{a}, \mathrm{Al}_{b}$, and $\mathrm{Al}_{c}$ are placed in contact with the complexing agent ferron (7-iodo-8-hydroxyquinoline-5-sulfonic acid) under mildly acidic conditions, the $\mathrm{Al}_{\mathrm{a}}$ reacts completely in less than 1 minute, and the $\mathrm{Al}_{\mathrm{b}}$ reacts in a relatively slow first-order reaction having a half-time of 5-20 minutes. The $\mathrm{Al}_{\mathrm{c}}$ form does not readily react with the ferron and is stable for several days in this environment.

Solutions were prepared by addition of differing amounts of hydroxide to solutions of $\mathrm{Al}^{+3}$, so that they were supersaturated in $\mathrm{Al}_{\mathrm{a}}$ with respect to gibbsite. The solutions approached equilibrium over several years aging as the polymeric $\mathrm{Al}_{\mathrm{b}}$ species grew in size. This process lowered the $\mathrm{pH}$, because the units lost hydrogen ions from some of the attached water molecules when hydroxide bridges were formed. The $\mathrm{Al}_{\mathrm{a}}$ concentration remained relatively constant during aging; eventually the $\mathrm{pH}$ decreased to an equilibrium value. Between age times of 10-20 and 100-120 days, the disappearance of $\mathrm{Al}_{\mathrm{b}}$ (to form $\mathrm{Al}_{\mathrm{c}}$ ) appeared to obey a first-order rate law.

The experimental aging-study solutions used by Smitr. and Hem (1972) constituted a relatively simple system in comparison to the aluminum hydroxide species occurring in ground or surface water systems. The aluminum hydroxide species were studied separately and apart from other complicating factors such as the effects on dissolved aluminum of dissolved silica, fluoride, sulfate, and organic ligands, and of various types of mineral surfaces. These factors are being considered in other papers of the series "Chemistry of Aluminum in Natural Water."

The purpose of this paper is to describe the effects of representative mineral surfaces on the behavior of dissolved and suspended aluminum hydroxide species, especially with regard to adsorption of dissolved aluminum species by the surfaces and their effect on the rate of polymerization of $\mathrm{Al}_{b}$ to $\mathrm{Al}_{c}$.

The general approach used in this investigation was to duplicate some of the experimental solutions prepared by Smith and Hem (1972), but with various known quantities of relatively insoluble minerals added in order to provide solid surfaces. Comparison of the results with those obtained in the absence of such surfaces permits a quantitative evaluation of their effects on the chemical ractions of aluminum that occur in systems moderately supersaturated with respect to gibbsite.

\section{ACKNOWLEDGMENTS}

Reviews of this paper by Professors R. W. Smith, University of Nevada (Reno) and G. A. Parks, Stanford University, were helpful in 
developing the presentation and are acknowledged with thanks. We also wish to thank C. J. Lind for furnishing all the electron micrographs used in the report.

\section{EXPERIMENTAL PLAN AND METHODS}

The work was begun by preparing a series of solutions containing the same total concentration of aluminum $\left(4.53 \times 10^{-4}\right.$ molar $)$ but different amounts of added base. These aging-study solutions were prepared by a technique identical to that described by Smith and Hem (1972). The $\mathrm{OH}: \mathrm{Al}$ ratio $\left(r_{n}\right)$ in the solutions as made up varied from 1.40 to 2.76 . The four solutions prepared were designated D, F, G, and $\mathrm{H}$ and were closely comparable with similarly designated solutions studied by Smith and Hem.

Smith and Hem (1972) found that the rate of addition of base in preparation of the aging-study solutions had a marked effect on the amount of $\mathrm{Al}_{\mathrm{b}}$ and $\mathrm{Al}_{\mathrm{c}}$ initially formed. $\mathrm{Al}_{\mathrm{b}}$ concentration was found to decrease and $\mathrm{Al}_{\mathrm{c}}$ concentration increase when the rate of base addition increased. $\mathrm{Al}_{\mathrm{a}}$ concentration was found to be independent of this rate. The slower rates of base addition thus yielded solutions with a higher $\mathrm{pH}$ and farther from equilibrium than those obtained by fast addition of base. In this set of experiments, base was added dropwis? over a period of 90-120 minutes in order to start the solutions with as much $\mathrm{Al}_{\mathrm{b}}$ and as little $\mathrm{Al}_{\mathrm{c}}$ and as far from equilibrium as practicable.

Each $3,000 \mathrm{ml}$ (millilitres) solution (D, F, G, and $\mathrm{H}$ ) was divided into 12 aliquots of $250 \mathrm{ml}$ each and placed in polyethylene bottles in which measured amounts of the various surfaces had been placed. Tl us from solution D were formed the following 12 solutions:

\begin{tabular}{|c|c|c|c|}
\hline $\begin{array}{l}\text { Amount and type } \\
\text { of surface }\end{array}$ & Abbreviation & $\begin{array}{l}\text { Added surface } \\
\qquad\left(m^{2}\right)\end{array}$ & $\begin{array}{c}\text { Surface per volume } \\
\left(\mathrm{m}^{2} / l\right)\end{array}$ \\
\hline No added surface (a) & D NASA & 0.0 & 0.0 \\
\hline No added surface $(b)$ & D NASB & .0 & .0 \\
\hline $0.010 \mathrm{~g}$ Wyoming bentonite & D $0.01 \mathrm{WB}$ & 5.5 & 22 \\
\hline $0.10 \mathrm{~g}$ Wyoming bentonite & D $0.1 \mathrm{WB}$ & 55 & 220 \\
\hline $0.20 \mathrm{~g}$ Wyoming bentonite & D $0.2 \mathrm{WB}$ & 110 & 440 \\
\hline $0.020 \mathrm{~g}$ volcanic ash & D $0.02 \mathrm{VA}$ & 2.2 & 8.8 \\
\hline $0.10 \mathrm{~g}$ volcanic ash & D $0.1 \mathrm{VA}$ & 11 & 44 \\
\hline $0.50 \mathrm{~g}$ volcanic ash $\ldots$ & $\mathrm{D} 0.5 \mathrm{VA}$ & 55 & 220 \\
\hline $0.10 \mathrm{~g}$ kaolinite \#17 & $\mathrm{D} 0.1 \mathrm{~K}$ & 1.5 & 6.0 \\
\hline $0.50 \mathrm{~g}$ kaolinite \# 17 & $\mathrm{D} 0.5 \mathrm{~K}$ & 7.5 & 30 \\
\hline $2.0 \mathrm{~g}$ kaolinite \# 17 & D $2.0 \mathrm{~K}$ & 30 & 120 \\
\hline $1.0 \mathrm{~g}$ Monterey sand & D $1.0 \mathrm{MS}$ & .5 & 2.0 \\
\hline
\end{tabular}

Solutions F, G, and $\mathrm{H}$ were similarly apportioned.

Each of these 48 solutions was analyzed for aqueous $\mathrm{Al}_{\mathrm{a}}$ and $\mathrm{Al}_{\mathrm{b}}$ in the manner described by Smith and Hem (1972). The aliquots used for 
analysis were centrifuged at 2,000 G's for 1 minute in order to remove particulate suspended material that might interfere with the spectrophotometric measurements. A modification of the ferron-orthophenanthroline analytical method for aluminum (Brown and others, 1970) was used in which the $\mathrm{pH}$ of sample aliquots was n€ver allowed to go below 5.0. Under these conditions, $\mathrm{Al}_{\mathrm{a}}$ reacts immediately with the ferron, while $\mathrm{Al}_{\mathrm{b}}$ reacts by first-order kinetics with ferron as these polymer species are attacked and dismantled by the acid. The halftime of the reaction ranges from 5 to 20 minutes, probably depending on the characteristic sizes and shapes of the $\mathrm{Al}_{\mathrm{b}}$ polymers encountered. In any event, the reaction is at least 95 percent completed after 90 minutes of analysis time. $\mathrm{Al}_{\mathrm{c}}$ does not react at a significant rate at $\mathrm{pH}$ 5. At a given analysis time, the amount of ferron-aluminum complex formation is determined spectrophotometrically at 370 nanometres. The concentration of $\mathrm{Al}_{\mathrm{b}}$ present at zero analysis time is determined by extrapolation of the first-order reaction rate to zero time using a plot of analysis time versus the negative logarithm of aluminum concentration recovered. The intercept is the negative logarithm of the analyzed $\mathrm{Al}_{\mathrm{b}}$ concentration $\left(\mathrm{pAl} \mathrm{b}_{\mathrm{b}}\right.$ ). $\mathrm{Al}_{\mathrm{a}}$ concentration is found by subtracting $\mathrm{Al}_{\mathrm{b}}$ concentration from the total concentration recovered after 90 minutes. The remainder of aluminum not recovered is aqueous and $\varepsilon$ dsorbed $\mathrm{Al}_{\mathrm{c}}$ and also adsorbed $\mathrm{Al}_{\mathrm{a}}$ and $\mathrm{Al}_{\mathrm{b}}$. At the same time as the aliquots for aluminum determinations were withdrawn, $\mathrm{pH}$ measurements were made using a Corning model $12 \mathrm{pH}$ meter, ${ }^{1}$ accurate to $\pm 0.01 \mathrm{pH}$ unit.

These $\mathrm{Al}_{\mathrm{a}}, \mathrm{Al}_{\mathrm{b}}$, and $\mathrm{pH}$ measurements were repeated at intervals of 1-3 days during the first 20 days of aging and at 5-15 day intervals after that. After the final analysis near the 100th day, dissolved silica concentrations were determined for each solution in order to evaluate the extent to which the added mineral surfaces might have undergone chemical attack and released solutes that influenced the processes of aluminum hydroxide polymerization.

\section{CHARACTERIZATION OF SURFACES}

The mineral materials used were selected to represent commonly occurring types similar to those that might be associated with natural water but more specifically definable than most natural soil or sediment. Two clays, kaolinite and montmorillonite, were used, and coarser grained material included volcanic ash and a feldspathic sand. The solids and their pretreatment are described subsequently.

The Wyoming bentonite (WB) was from a sample collected in January 1965 at Belle Fourche, S. Dak. It was dried at $90^{\circ} \mathrm{C}$ for 10

${ }^{1}$ Use of trade names or commercial products in this report is for identification only and does not constitute endorsement by the U.S. Geological Survey. 
minutes, then roller crushed to particle sizes of $<200$ mesh. Dr: sieve analysis was 90 percent $<20 \mu \mathrm{m}$, while wet analysis showed 99 rercent $<20 \mu \mathrm{m}$ and 92 percent $<0.5 \mu \mathrm{m}$.

Ward's standard volcanic ash (New Mexico) fragments (VA) were ground in a mortar and pestle until all particles could pass through a 32-mesh sieve, were thrice washed in distilled water, and then d "ied at $90^{\circ} \mathrm{C}$. Dry sieve analysis showed 100 percent $<500 \mu \mathrm{m}, 67$ rercent $<250 \mu \mathrm{m}, 48$ percent $<150 \mu \mathrm{m}, 42$ percent $<125 \mu \mathrm{m}, 35$ percent $<105 \mu \mathrm{m}, 19$ percent $<74 \mu \mathrm{m}$, and 12 percent $<44 \mu \mathrm{m}$. Microscopic examination showed most of the particles to have irregular jagged surfaces to which no simple geometric model for evaluating surface areas could be applied.

Ward's standard kaolinite \#17 (Lewistown, Montana) (K) was treated by the same method as that used on volcanic ash. No sieve analysis was performed.

Monterey sand, a beach sand from Monterey, Calif. (MS), was obtained from the Braun-Knecht-Heimann Company, San Francisco, Calif. It was twice washed with 35 percent $\mathrm{Na}_{2} \mathrm{SO}_{3}$ solution at $\mathrm{pH} 3$ in order to remove $\mathrm{MnO}_{2}$ formed on the sand surface in previous experimental studies by Hem (1964), who reported 55 percent of the grains were feldspar and 45 percent quartz. The grains were moderately rounded, and 85 percent of them were between 0.25 and $0.8 \mathrm{~mm}$ (millimetre) in diameter. None were larger than 0.8 or smaller than $0.1 \mathrm{~mm}$.

The specific areas of the surfaces used were determined by the orthophenanthroline and ethylene glycol monolayer adsorption methods by Lawrie (1961) and Bower and Goertzen (1959), respectively. The orthophenanthroline adsorbed by a known amount of solid was determined by subtracting the concentration remaining in a solution shaken with the solid from the concentration of the saturated orthophenanthroline solution used. Analyses were made colorimetrically using the iron (II) orthophenanthroline complex. Specific surface areas obtained by this method are as follows:

Wyoming bentonite

Volcanic ash

Kaolinite \#17

Monterey sand
$550 \pm 40 \mathrm{~m}^{2} / \mathrm{g}$ (metres squared per gram)

$110 \pm 10 \mathrm{~m}^{2} / \mathrm{g}$

$15 \pm 2 \mathrm{~m}^{2} / \mathrm{g}$

$0.5 \pm 0.05 \mathrm{~m}^{2} / \mathrm{g}$

Ethylene glycol adsorbed by a known amount of solid was determined gravimetrically. Values obtained using this method are as follows:

Wyoming bentonite

Volcanic ash

Kaolinite \#17
$550 \pm 110 \mathrm{~m}^{2} / \mathrm{g}$

$118 \pm 10 \mathrm{~m}^{2} / \mathrm{g}$

$17 \pm 2 \mathrm{~m}^{2} / \mathrm{g}$ 
Cation exchange capacities of the surfaces used were determined using the method of Chapman (1965). By this procedure the clay or soil is saturated with sodium acetate, then ammonium acetate is used to replace the sodium which is then analyzed by atomic absorption spectrophotometry. Values obtained are as follows:

$\begin{array}{lr}\text { Wyoming bentonite } & 922 \mu \mathrm{eq} / \mathrm{g} \text { (microequivalents per gram) } \\ \text { Volcanic ash } & 69 \mu \mathrm{eq} / \mathrm{g} \\ \text { Kaolinite \#17 } & 6.6 \mu \mathrm{eq} / \mathrm{g} \\ \text { Monterey sand }\end{array}$

Determinations of the point of zero charge were made by measuring adsorption of hydrogen or hydroxide using the titration method described by T. W. Healy (oral commun., 1970), and the results obtained are as follows:

Wyoming bentonite

$\mathrm{pH} 8.1$

Volcanic ash

Kaolinite \#17

4.3

Cation exchange capacity for the four materials studied is closely related to surface area, as shown by the plot of these data (fig. 1). The degree to which this relationship may be more generally applicable is uncertain, however. For one thing, the two properties, exchange capacity and surface area, were measured by chemically similar techniques. The results thus have an internal bias that might lead to a relationship of this type. However, the wet adsorption procedures for surface area gave results that were in approximate agreement witl a few measurements on the same materials made by the Brunauer-EmmettTeller (BET) procedure. The surfaces of all four materials are similar in the sense that all are dominated by oxygen ions bonded to silicon, and some similarity in behavior should not be surprising. Surfaces with radically different structure would perhaps behave differently from the ones studied.

Electron micrographs of the Wyoming bentonite, volcanic ash, and kaolinite materials used in this study are given as fig ures 2-4. The thin plates of montmorillonite are well displayed in the kentonite. The volcanic ash is composed of irregular glassy shards without a well-defined crystal structure. The kaolinite consists me inly of rather large layered plates with the angular outline typical of this mineral.

\section{LONG-TERM AGING EFFECTS}

The solutions studied by Smith and Hem (1972) were analyzed at intervals over about 9 months, and their paper also included some data on equivalent solutions aged as much as 3 years. Most of the changes 


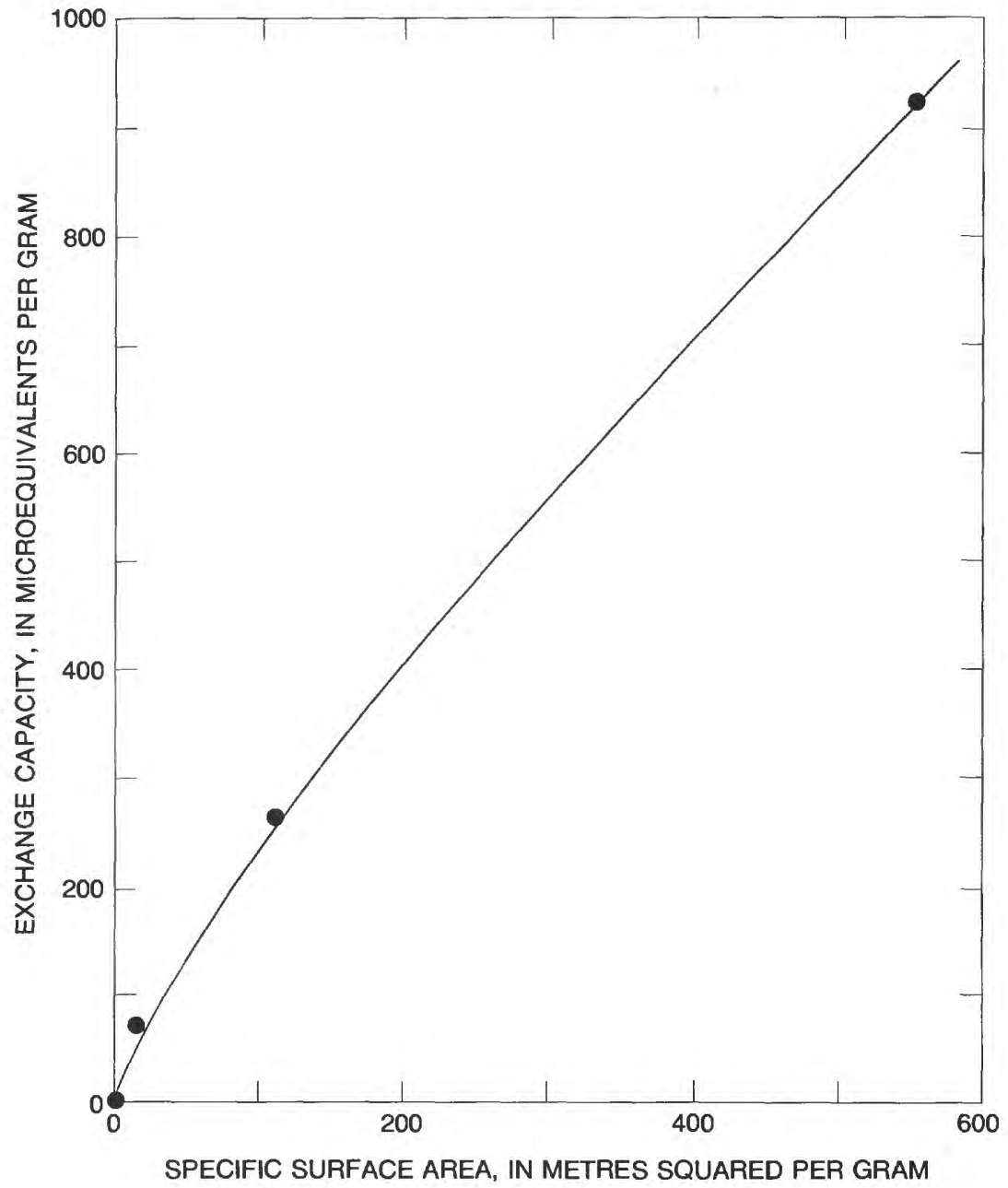

FIGURE 1.-Relation between specific surface area and cation exchange capacity for materials studied.

occurred in the first 4 months of study, however, and it therefore appeared possible to evaluate adequately the influence of the surfaces on the attainment of equilibrium solubility of aluminum with a maximum of 4 months of aging of the test solutions. The results of these experiments and factors considered in their interpretation follow. Observed $\mathrm{pH}$ and concentrations of aluminum species for each solution are given in tables $1-4$.

\section{INTERPRETATION OF RESULTS}

The concentrations of dissolved species that can be measured, $\mathrm{Al}_{\mathrm{a}}$, 


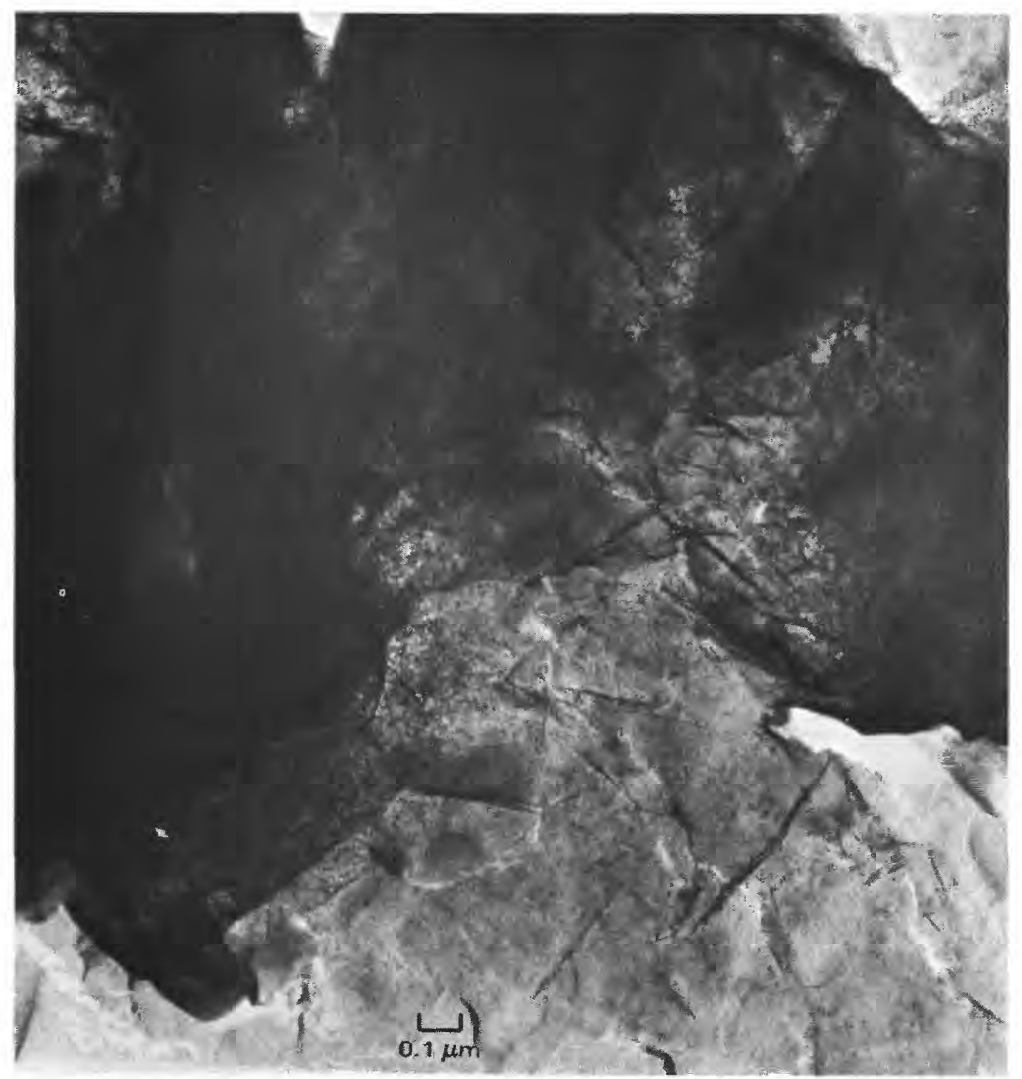

FIGURE 2.-Electron micrograph of Wyoming bentonite.

$\mathrm{Al}_{\mathrm{b}}$, and $\mathrm{H}^{+}$, constitute the information that is available about the processes that occurred. Where correlated with what is known about the mineral surfaces, some insight into the nature of the processes ought to be possible. Several alternative hypotheses can be suggested as a starting point for the interpretation of the experimental data. The following are possible conditions and ways for evaluating them.

1. Chemical reactions may have occurred in which the surfaces were actually attacked and partly brought into solution. Such reactions might have produced a new solid aluminosilicate whose behavior would affect aluminum solubility in a way different from simpler aluminum hydroxide species. The extent of attack on the surfaces can be evaluated by determining dissolved silica in the aged solutions. A comparison of the halloysite ion activity products calculated from $\mathrm{Si}(\mathrm{OH})_{4}^{0}, \mathrm{Al}^{+3}$, and $\mathrm{H}^{+}$activities in these solutions with those observed by Hem, Roberson, Lind, and Polzer (1973) for such material should indicate the importance of 


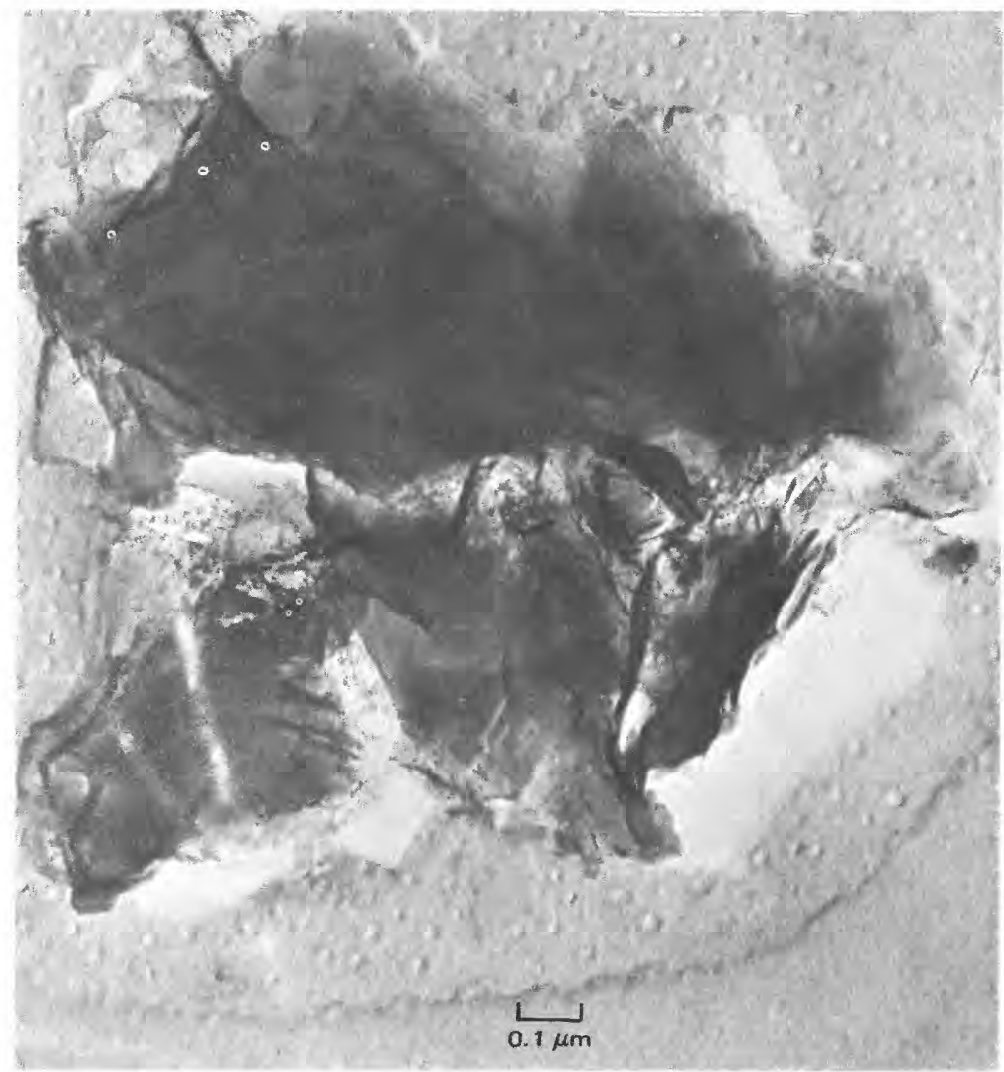

Figure 3.-Electron micrograph of volcanic ash.

reactions involving surface dissolution. If some of the solutions appear to be producing an aluminosilicate solid they should not be included in an evaluation of equilibria involving gibbsite.

2. Mineral surfaces catalyze or facilitate the processes of polymerization of aluminum hydroxide previously observed. Essentially this involves the conversion of $\mathrm{Al}_{b}$ to $\mathrm{Al}_{c}$ (gibbsite). It should be feasible to evaluate this possibility by comparing rates of dissappearance of $\mathrm{Al}_{\mathrm{b}}$ and testing for adherence to gibbsite solubility equilibria or by observing the rate and extent of $\mathrm{pH}$ shift in the presence or absence of surfaces.

3. Mineral surfaces alter concentrations of cations in solution by chemisorption. This can be studied by applying cation exchange equilibria.

4. Physical adsorption of polymerized species of low surface charge density occurs. The behavior of $\mathrm{Al}_{\mathrm{b}}$ and $\mathrm{Al}_{\mathrm{c}}$ might be so affected and can be explored by surface adsorption models. 


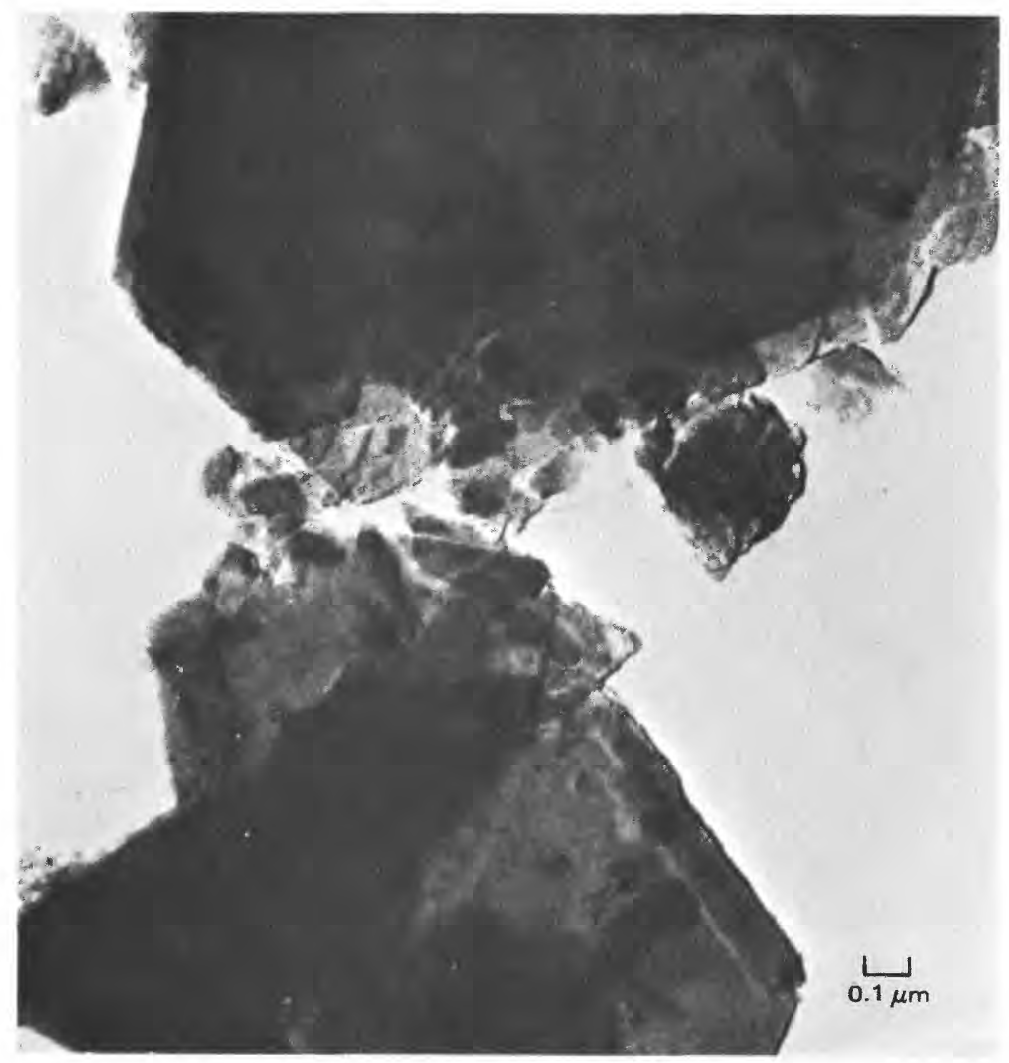

FigURE 4.-Electron micrograph of kaolinite.

A combination of certain of these processes may well occur. From a systematic examination of the experimental data, however, it should be possible to decide which processes predominate. In addition to the chemical data, electron micrographs of solids before and after exposure to aluminum solutions are available. These provide qualitative evidence of crystallization processes at the surfaces.

\section{POSSIBILITY OF ALUMINOSILICATE FORMATION}

As pointed out earlier, if the mineral surface in a particular solution were attacked and partly dissolved, this could be detected by the presence of aqueous silica. Studies by Hem, Roberson, Lind, and Polzer (1973, p. 16) indicate that solutions containing aluminum in concentrations similar to the ones studied here, with $2.0 \mathrm{mg} / 1$ (milligrams per litre) $\mathrm{SiO}_{2}$ or less, precipitated only crystalline $\mathrm{Al}(\mathrm{OH})_{3}$. Where more silica was present, there was a mixed product; 
TABLE 1.-Concentrations of two types of dissolved aluminum and $\mathrm{pH}$ in relation to age and amount and type of mineral surface in solutions $D$

[Asterisks indicate solutions which attacked surfaces]

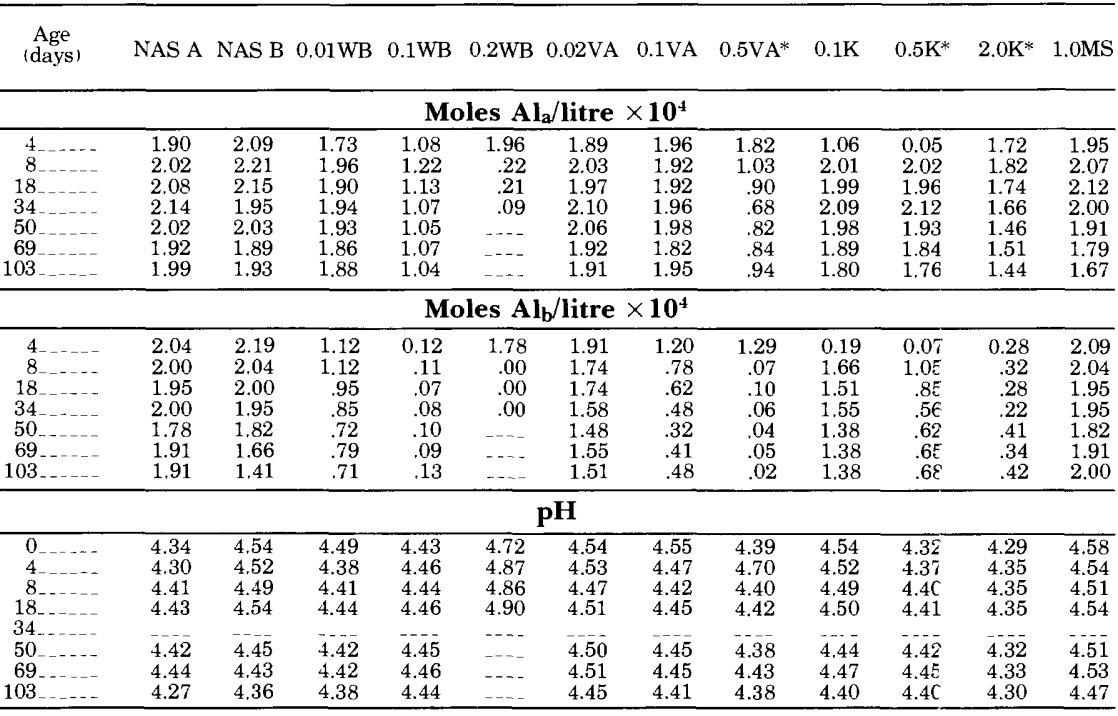

TABLE 2.-Concentrations of two types of dissolved aluminum and $\mathrm{pH}$ in rolation to age and amount and type of mineral surface in solutions $F$

[Asterisks indicate solutions which attacked surfaces]

\begin{tabular}{|c|c|c|c|c|c|c|c|c|c|c|c|c|}
\hline $\begin{array}{c}\text { Age } \\
\text { (days) }\end{array}$ & NAS A & NAS B & $0.01 \mathrm{WB}$ & $0.1 \mathrm{WB}^{*}$ & $0.2 \mathrm{WB}^{*}$ & $0.02 \mathrm{VA}$ & $0.1 \mathrm{VA}$ & $0.5 \mathrm{VA}$ & $0.1 \mathrm{~K}$ & $0.5 \mathrm{~K}^{*}$ & $2.0 \mathrm{~K}^{\star}$ & 1.0MS \\
\hline \multicolumn{13}{|c|}{ Moles $\mathrm{Al}_{\mathrm{a}} /$ litre $\times 10^{4}$} \\
\hline $\begin{array}{r}2 \ldots \\
3 \ldots \ldots \\
6 \ldots \ldots \\
12 \ldots \ldots \\
19 \ldots \ldots \\
32 \ldots \ldots \\
45 \ldots \ldots \\
69 \ldots \ldots \\
82 \ldots \ldots \\
98 \ldots \ldots\end{array}$ & $\begin{array}{r}1.25 \\
.85 \\
.95 \\
.97 \\
1.02 \\
.88 \\
.95 \\
1.18 \\
1.00 \\
1.21\end{array}$ & $\begin{array}{r}0.95 \\
.86 \\
.92 \\
.97 \\
.97 \\
1.16 \\
1.06 \\
1.20 \\
\ldots .--\end{array}$ & $\begin{array}{r}1.49 \\
.92 \\
.99 \\
.90 \\
.88 \\
1.06 \\
.95 \\
1.16 \\
1.09 \\
1.14\end{array}$ & $\begin{array}{r}0.00 \\
.22 \\
.27 \\
.16 \\
.19 \\
.18 \\
.20 \\
.20 \\
-.-\end{array}$ & $\begin{array}{r}0.09 \\
.07 \\
.00 \\
.35 \\
.02 \\
.25 \\
.04 \\
.24 \\
-.-- \\
-. .-\end{array}$ & $\begin{array}{r}0.97 \\
.82 \\
1.04 \\
.98 \\
.20 \\
.98 \\
.96 \\
1.27 \\
1.00 \\
1.25\end{array}$ & $\begin{array}{r}0.84 \\
.69 \\
.97 \\
.98 \\
.85 \\
.95 \\
.90 \\
1.18 \\
.88 \\
1.06\end{array}$ & $\begin{array}{r}0.50 \\
.25 \\
.00 \\
.16 \\
.12 \\
.11 \\
.07 \\
.19 \\
-.- \\
-.--\end{array}$ & $\begin{array}{r}1.07 \\
1.13 \\
1.07 \\
.98 \\
.83 \\
.91 \\
1.01 \\
1.17 \\
1.08 \\
1.26\end{array}$ & $\begin{array}{r}1.61 \\
1.5 \mathrm{C} \\
1.0 \mathrm{C} \\
.98 \\
.98 \\
.9 \varepsilon \\
.94 \\
1.11 \\
.8 \varepsilon \\
1.1 \AA\end{array}$ & $\begin{array}{r}1.44 \\
1.29 \\
.66 \\
.76 \\
.82 \\
.79 \\
.80 \\
.86 \\
.63 \\
.80\end{array}$ & $\begin{array}{r}0.93 \\
.66 \\
.91 \\
.92 \\
.85 \\
.93 \\
.93 \\
1.25 \\
.97 \\
1.19\end{array}$ \\
\hline \multicolumn{13}{|c|}{ Moles $\mathrm{Al}_{\mathrm{b}} /$ litre $\times 10^{4}$} \\
\hline 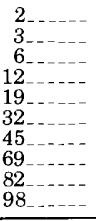 & $\begin{array}{r}3.02 \\
2.88 \\
2.75 \\
2.57 \\
2.19 \\
1.66 \\
1.17 \\
.59 \\
.38 \\
.32 \\
\end{array}$ & $\begin{array}{r}2.88 \\
2.95 \\
2.81 \\
2.57 \\
2.14 \\
1.58 \\
1.05 \\
.49 \\
--. \overline{2} \\
\end{array}$ & $\begin{array}{r}2.14 \\
2.29 \\
2.09 \\
1.82 \\
1.74 \\
1.29 \\
1.05 \\
.51 \\
.35 \\
-.-- \\
\end{array}$ & $\begin{array}{r}0.43 \\
.04 \\
.00 \\
.02 \\
.05 \\
.02 \\
.00 \\
.00 \\
-.-- \\
-.-- \\
\end{array}$ & $\begin{array}{r}0.06 \\
.03 \\
.02 \\
.10 \\
.07 \\
.02 \\
.00 \\
.00 \\
-. .- \\
.-.- \\
\end{array}$ & $\begin{array}{r}2.51 \\
2.69 \\
2.57 \\
2.34 \\
1.45 \\
1.29 \\
.85 \\
.25 \\
.16 \\
.10\end{array}$ & $\begin{array}{r}1.66 \\
1.70 \\
1.48 \\
1.35 \\
1.20 \\
.85 \\
.56 \\
.09 \\
.08 \\
.10 \\
\end{array}$ & $\begin{array}{r}1.38 \\
.04 \\
1.15 \\
.05 \\
.04 \\
.02 \\
.01 \\
.02 \\
-.-- \\
-.-- \\
\end{array}$ & $\begin{array}{r}2.45 \\
2.63 \\
2.45 \\
2.19 \\
2.14 \\
1.51 \\
1.17 \\
.60 \\
.37 \\
.26 \\
\end{array}$ & $\begin{array}{l}1.5 \varepsilon \\
1.76 \\
1.45 \\
1.26 \\
1.2 \varsigma \\
1.0 \AA \\
.9 \varepsilon \\
.71 \\
.62 \\
.52\end{array}$ & $\begin{array}{r}0.21 \\
.15 \\
.21 \\
.10 \\
.10 \\
.06 \\
.03 \\
.04 \\
.07 \\
.04 \\
\end{array}$ & $\begin{array}{l}2.75 \\
2.88 \\
2.69 \\
2.51 \\
2.23 \\
1.58 \\
1.10 \\
.58 \\
.38 \\
.24 \\
\end{array}$ \\
\hline \multicolumn{13}{|c|}{ pH } \\
\hline $\begin{array}{r}0 \ldots \ldots \\
2 \\
3 \\
6 \\
12 \\
19 \\
32 \\
45 \\
69 \\
82 \\
98\end{array}$ & $\begin{array}{l}4.75 \\
4.65 \\
4.64 \\
4.56 \\
4.57 \\
4.48 \\
4.03 \\
4.38 \\
4.04 \\
4.25 \\
4.29\end{array}$ & $\begin{array}{l}4.75 \\
4.65 \\
4.48 \\
4.59 \\
4.57 \\
4.48 \\
4.29 \\
4.38 \\
4.25 \\
4.40\end{array}$ & $\begin{array}{l}4.74 \\
4.65 \\
4.64 \\
4.62 \\
4.58 \\
4.48 \\
4.47 \\
4.45 \\
4.38 \\
4.38 \\
-. .-\end{array}$ & $\begin{array}{l}4.69 \\
4.73 \\
4.70 \\
4.67 \\
4.78 \\
4.66 \\
4.72 \\
4.75 \\
4.72 \\
-.- \\
-\end{array}$ & $\begin{array}{l}5.33 \\
5.36 \\
5.39 \\
5.38 \\
5.53 \\
5.27 \\
5.41 \\
5.36 \\
5.35 \\
-\ldots- \\
-\ldots-\end{array}$ & $\begin{array}{l}4.75 \\
4.69 \\
4.65 \\
4.60 \\
4.59 \\
4.28 \\
4.43 \\
4.42 \\
4.33 \\
4.31 \\
4.30\end{array}$ & $\begin{array}{l}4.75 \\
4.68 \\
4.64 \\
4.61 \\
4.58 \\
4.52 \\
4.49 \\
4.46 \\
4.41 \\
4.38 \\
4.31\end{array}$ & $\begin{array}{l}4.68 \\
4.74 \\
4.74 \\
4.80 \\
4.79 \\
4.76 \\
4.76 \\
4.68 \\
4.85 \\
--- \\
-.-\end{array}$ & $\begin{array}{l}4.76 \\
4.67 \\
4.66 \\
4.63 \\
4.61 \\
4.60 \\
4.48 \\
4.44 \\
4.41 \\
4.40 \\
4.38\end{array}$ & $\begin{array}{l}4.70 \\
4.65 \\
4.63 \\
4.62 \\
4.60 \\
4.63 \\
4.52 \\
4.53 \\
4.54 \\
4.52 \\
4.54\end{array}$ & $\begin{array}{l}4.53 \\
4.55 \\
4.54 \\
4.55 \\
4.51 \\
4.63 \\
4.47 \\
4.45 \\
4.45 \\
4.43 \\
4.48\end{array}$ & $\begin{array}{l}4.76 \\
4.72 \\
4.67 \\
4.67 \\
4.60 \\
4.48 \\
4.43 \\
4.40 \\
4.36 \\
4.34 \\
4.35\end{array}$ \\
\hline
\end{tabular}


TABLE 3-Concentrations of two types of dissolved aluminum and $\mathrm{pH}$ in relation to age and amount and type of mineral surface in solutions $G$

[Asterisks indicate solutions which attacked surfaces]

\begin{tabular}{|c|c|c|c|c|c|c|c|c|c|c|c|c|}
\hline $\begin{array}{l}\text { Age } \\
\text { (days) }\end{array}$ & NAS A & NAS B & $0.01 \mathrm{WB}$ & $0.1 \mathrm{WB}$ & $0.2 \mathrm{WB}^{5}$ & $0.02 \mathrm{VA}$ & $0.1 \mathrm{VA}$ & $0.5 \mathrm{VA}$ & $0.1 \mathrm{~K}$ & $0.5 \mathrm{~F}$ & $2.0 \mathrm{~K}^{*}$ & $1.0 \mathrm{MS}$ \\
\hline \multicolumn{13}{|c|}{ Moles $\mathrm{Al}_{\mathrm{a}} /$ litre $\times 10^{4}$} \\
\hline $\begin{array}{r}1 \\
3 \\
7 \\
14 \ldots \\
25 \\
51 \ldots \\
64 \ldots \\
85 \\
95\end{array}$ & $\begin{array}{r}0.24 \\
.15 \\
.13 \\
.24 \\
.31 \\
.54 \\
.55 \\
.59 \\
.70\end{array}$ & $\begin{array}{r}0.23 \\
.19 \\
.17 \\
.32 \\
.34 \\
.74 \\
.60 \\
.58 \\
.98\end{array}$ & $\begin{array}{r}0.17 \\
.20 \\
.14 \\
.22 \\
.31 \\
.49 \\
.42 \\
.51 \\
.57\end{array}$ & $\begin{array}{r}0.02 \\
.04 \\
.02 \\
.08 \\
.04 \\
.09 \\
-.- \\
-.-\end{array}$ & $\begin{array}{r}0.00 \\
.00 \\
.05 \\
.08 \\
.08 \\
.18 \\
- \\
-.-- \\
-.\end{array}$ & $\begin{array}{l}0.18 \\
.13 \\
.20 \\
.24 \\
.34 \\
.63 \\
.57 \\
.78 \\
.61\end{array}$ & $\begin{array}{r}0.19 \\
.15 \\
.21 \\
.32 \\
.33 \\
.46 \\
.48 \\
.55 \\
-.--\end{array}$ & $\begin{array}{r}0.00 \\
.00 \\
.00 \\
.00 \\
.00 \\
.07 \\
.00 \\
.00\end{array}$ & $\begin{array}{r}0.21 \\
.17 \\
.18 \\
.32 \\
.34 \\
.53 \\
.53 \\
.59 \\
.66\end{array}$ & $\begin{array}{r}0.29 \\
.29 \\
.29 \\
.33 \\
.31 \\
.50 \\
.38 \\
.40 \\
.52\end{array}$ & $\begin{array}{r}0.31 \\
.33 \\
.23 \\
.32 \\
.25 \\
.27 \\
\ldots . \\
-.-\end{array}$ & $\begin{array}{r}0.30 \\
.24 \\
.21 \\
.25 \\
.30 \\
.45 \\
.49 \\
60 \\
.43\end{array}$ \\
\hline \multicolumn{13}{|c|}{ Moles $\mathrm{Al}_{\mathrm{b}} /$ litre $\times 10^{4}$} \\
\hline $\begin{array}{r}1 \\
3 \\
7 \\
14 \\
25 \\
51 \\
64 \\
85 \\
95\end{array}$ & $\begin{array}{l}2.88 \\
2.81 \\
2.69 \\
2.29 \\
1.74 \\
.83 \\
.56 \\
.32 \\
.20\end{array}$ & $\begin{array}{r}2.88 \\
2.75 \\
2.63 \\
240 \\
1.74 \\
.83 \\
56 \\
.30 \\
.22\end{array}$ & $\begin{array}{l}2.19 \\
2.14 \\
2.09 \\
1.86 \\
1.51 \\
.74 \\
.50 \\
.23 \\
.17\end{array}$ & $\begin{array}{r}0.01 \\
.01 \\
.00 \\
.01 \\
.01 \\
.00 \\
-.- \\
-.- \\
-.-\end{array}$ & $\begin{array}{r}0.04 \\
.01 \\
.00 \\
.03 \\
.00 \\
.02 \\
-.- \\
-. .- \\
-.-\end{array}$ & $\begin{array}{r}2.63 \\
2.63 \\
2.40 \\
2.19 \\
1.51 \\
.62 \\
.38 \\
.14 \\
.13\end{array}$ & $\begin{array}{r}1.91 \\
1.70 \\
1.35 \\
1.15 \\
.85 \\
.21 \\
.01 \\
.00 \\
-.-\end{array}$ & $\begin{array}{l}0.15 \\
.02 \\
.00 \\
.00 \\
.00 \\
.01 \\
.01 \\
.00\end{array}$ & $\begin{array}{l}2.63 \\
2.51 \\
2.40 \\
2.23 \\
1.74 \\
.89 \\
.63 \\
.37 \\
.23\end{array}$ & $\begin{array}{l}1.95 \\
1.78 \\
1.66 \\
1.48 \\
1.29 \\
.93 \\
.79 \\
.74 \\
.62\end{array}$ & $\begin{array}{r}0.41 \\
.19 \\
.06 \\
.02 \\
.00 \\
.01 \\
-.-- \\
-.-- \\
-.-\end{array}$ & $\begin{array}{l}2.75 \\
2.69 \\
2.51 \\
2.34 \\
1.74 \\
.85 \\
.59 \\
.34 \\
.30\end{array}$ \\
\hline \multicolumn{13}{|c|}{$\mathbf{p H}$} \\
\hline $\begin{array}{r}0 \ldots \ldots \\
1 \ldots \ldots \\
3 \\
7 \\
14 \\
25 \\
51 \ldots \ldots \\
64 \ldots \ldots \\
85 \ldots \\
95\end{array}$ & $\begin{array}{l}4.92 \\
4.74 \\
4.81 \\
4.66 \\
4.61 \\
4.53 \\
4.34 \\
4.40 \\
4.41 \\
4.46 \\
\end{array}$ & $\begin{array}{l}4.95 \\
4.83 \\
4.91 \\
4.79 \\
4.68 \\
4.58 \\
4.47 \\
4.49 \\
4.42 \\
4.43 \\
\end{array}$ & $\begin{array}{l}4.94 \\
4.82 \\
4.93 \\
4.84 \\
4.76 \\
4.65 \\
4.56 \\
4.59 \\
4.58 \\
4.53 \\
\end{array}$ & $\begin{array}{l}5.84 \\
6.29 \\
6.25 \\
6.3 \\
6.49 \\
6.35 \\
5.97 \\
---- \\
--- \\
--- \\
\end{array}$ & $\begin{array}{l}6.40 \\
6.69 \\
6.76 \\
6.73 \\
6.92 \\
6.35 \\
6.65 \\
---- \\
---- \\
--- \\
\end{array}$ & $\begin{array}{l}4.96 \\
4.97 \\
4.86 \\
4.72 \\
4.60 \\
4.61 \\
4.51 \\
4.50 \\
4.44 \\
4.42 \\
\end{array}$ & $\begin{array}{l}4.96 \\
5.00 \\
4.90 \\
4.86 \\
4.76 \\
4.68 \\
4.59 \\
4.60 \\
4.55 \\
- \\
\end{array}$ & $\begin{array}{l}5.08 \\
5.38 \\
5.41 \\
5.48 \\
5.55 \\
5.40 \\
5.59 \\
5.75 \\
5.64 \\
----\end{array}$ & $\begin{array}{l}4.95 \\
4.93 \\
4.91 \\
4.86 \\
4.78 \\
4.70 \\
4.58 \\
4.57 \\
4.52 \\
4.48 \\
\end{array}$ & $\begin{array}{l}4.89 \\
4.72 \\
4.65 \\
4.83 \\
4.78 \\
4.73 \\
4.70 \\
4.74 \\
4.72 \\
4.67\end{array}$ & $\begin{array}{r}4.82 \\
4.80 \\
4.74 \\
4.77 \\
4.73 \\
4.69 \\
4.70 \\
-- \\
--- \\
\end{array}$ & $\begin{array}{l}4.96 \\
4.91 \\
4.87 \\
4.86 \\
4.69 \\
4.68 \\
4.51 \\
4.53 \\
4.48 \\
4.46 \\
\end{array}$ \\
\hline
\end{tabular}

however, silica concentrations as much as $9.0 \mathrm{mg} / 1 \mathrm{SiO}_{2}$ still gave solids that contained gibbsite identifiable by X-ray diffraction, and solubility data indicated a gibbsite equilibrium was reached. These olservations suggest that if concentrations of $\mathrm{SiO}_{2}$ remained below $9.0 \mathrm{mg} / \mathrm{l}$, gibbsite would be precipitated in solutions similar to the ones prepared here. Nonetheless, it is expedient to examine aluminosilicate equilibria in all the solutions where silica was detected even if the amount is less than $9.0 \mathrm{mg} / \mathrm{l}$, because higher levels might have been present in some of these systems at some time during the aging process.

To say whether a solution may be at equilibrium with respect to a silicate, we must first determine from the $\mathrm{H}^{+}, \mathrm{Na}^{+}, \mathrm{Al}^{+3}$, and $\mathrm{Si}(\mathrm{OH})_{4}$ activities whether various solutions are saturated or supersaturated with respect to possible aluminosilicates. It can be shown that within the ranges of these activities in our solutions the most likely minerals for which saturation or supersaturation would occur are halloysite and kaolinite, for which the equilibrium can be written

$$
1 / 2 \mathrm{Al}_{2} \mathrm{Si}_{2} \mathrm{O}_{5}(\mathrm{OH})_{4}+3 \mathrm{H}^{+} \rightleftharpoons \mathrm{Al}^{+3}+\mathrm{Si}(\mathrm{OH})_{4}+1 / 2 \mathrm{H}_{2} \mathrm{O}
$$


TABLE 4.-Concentrations of two types of dissolved aluminum and $\mathrm{pH}$ in relation to age and amount and type of mineral surface in solutions $H$

[Asterisks indicate solutions which attacked surfaces]

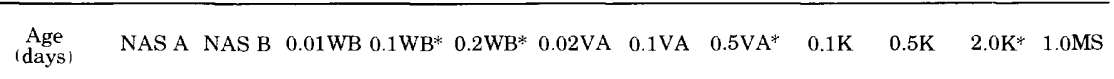

\begin{tabular}{|c|c|c|c|c|c|c|c|c|c|c|c|c|}
\hline \multicolumn{13}{|c|}{ Moles $\mathrm{Al}_{\mathrm{a}} /$ litre $\times 10^{4}$} \\
\hline $1 \ldots$ & 0.11 & 0.12 & 0.15 & 0.00 & 0.03 & 0.00 & 0.08 & 0.02 & 0.08 & 0.48 & 1.21 & 0.09 \\
\hline 3 & .02 & .12 & .21 & .31 & .12 & .09 & .06 & .10 & .52 & .14 & .00 & .05 \\
\hline 6 & .00 & .00 & .00 & .09 & .31 & .01 & .00 & .00 & .02 & .47 & .00 & .00 \\
\hline 12 & .09 & .08 & .08 & .04 & .00 & .08 & .12 & .00 & .12 & .14 & .04 & .13 \\
\hline 20 & .22 & .23 & .02 & .00 & .00 & .17 & .13 & .00 & .19 & .06 & .02 & .26 \\
\hline 32 & .19 & .31 & .16 & .00 & .03 & .19 & .22 & .00 & .22 & .15 & .02 & .21 \\
\hline 45 & .25 & .33 & .17 & .10 & .18 & .32 & .08 & .00 & .20 & .19 & .11 & .27 \\
\hline $72 \ldots$ & .46 & .54 & .27 & .11 & .23 & .49 & .27 & .09 & .32 & .27 & .05 & .44 \\
\hline $81 \ldots$ & .27 & .37 & .21 & $-\ldots$ & - - - & .44 & $\ldots-$ & $\ldots$ & .26 & .22 & $\ldots$ & .34 \\
\hline $97 \ldots$ & .48 & .42 & .34 & - - - & - - - & .46 & $\ldots$ & $\ldots$ & .31 & .30 & $\ldots$ & .40 \\
\hline
\end{tabular}

\begin{tabular}{|c|c|c|c|c|c|c|c|c|c|c|c|c|}
\hline \multicolumn{13}{|c|}{ Moles $\mathrm{Al}_{\mathrm{b}} /$ litre $\times 10^{4}$} \\
\hline 1. & 2.57 & 2.57 & 1.95 & 0.20 & 0.05 & 2.34 & 1.58 & 0.23 & 2.40 & 1.74 & 034 & 2.51 \\
\hline$\ldots$ & 2.57 & 2.57 & 2.04 & .19 & .19 & 2.23 & 1.29 & .17 & 2.34 & 1.41 & .08 & 2.45 \\
\hline $6 \ldots$ & 2.57 & 2.51 & 2.04 & .01 & .01 & 2.14 & 1.02 & .05 & 2.29 & 1.74 & .58 & 2.40 \\
\hline 12 & 2.09 & 2.14 & 1.41 & .05 & .03 & 1.82 & .78 & .07 & 1.86 & 1.23 & .05 & 1.91 \\
\hline $20 \ldots$ & 1.51 & 1.66 & 1.23 & .02 & .03 & 1.26 & .39 & .10 & 1.45 & 1.05 & .02 & 1.41 \\
\hline 32 & 1.05 & 1.15 & .89 & .08 & .02 & .85 & .12 & .02 & 1.50 & .87 & .03 & .98 \\
\hline $45 \ldots$ & .71 & .78 & .55 & .02 & .01 & .55 & .04 & .03 & .74 & .71 & .01 & .66 \\
\hline $72 \ldots$ & .31 & .38 & .19 & .01 & .14 & .19 & .10 & .02 & .32 & .50 & .02 & .30 \\
\hline $81 \ldots$ & .24 & .23 & .10 &.-- & $-\ldots$ & .11 & $-\ldots$ & $\ldots$ & .32 & .40 & $\ldots$ & .19 \\
\hline $97_{-\ldots-\ldots}$ & .12 & .14 & .02 & ---- & $\ldots$ & .03 & $\ldots$ & --- & .19 & .33 & $\ldots$ & .13 \\
\hline \multicolumn{13}{|c|}{$\mathbf{p H}$} \\
\hline 0 & 5.10 & 5.12 & 5.13 & 6.33 & 6.89 & 5.18 & 5.18 & 5.50 & 5.14 & 5.07 & 512 & 5.16 \\
\hline 1 & 5.19 & 5.31 & 5.39 & 6.59 & 6.53 & 5.33 & 5.45 & 6.09 & 5.40 & 5.25 & 519 & 5.36 \\
\hline $3 \ldots$ & 5.01 & 5.17 & 5.30 & 6.59 & 7.01 & 5.22 & 5.34 & 5.92 & 5.22 & 5.32 & 520 & 5.21 \\
\hline $6 \ldots$ & 5.06 & 5.08 & 5.10 & 6.45 & 6.45 & 5.18 & 5.22 & 5.90 & 5.18 & 5.12 & 509 & 5.17 \\
\hline $12 \ldots$ & 4.45 & 4.78 & 5.01 & 6.44 & 7.00 & 5.00 & 5.02 & 5.87 & 5.07 & 5.07 & 503 & 4.88 \\
\hline $20 \ldots$ & 4.40 & 4.50 & 4.83 & 6.63 & 7.03 & 4.75 & 4.93 & 5.75 & 4.87 & 4.99 & 501 & 4.82 \\
\hline $32 \ldots \ldots$ & 4.59 & 4.68 & 4.73 & 6.56 & 6.96 & 4.72 & 4.75 & 5.82 & 4.82 & 4.93 & 4.97 & 4.67 \\
\hline $45 \ldots$ & 4.54 & 4.63 & 4.68 & 6.45 & 6.85 & 4.64 & 4.70 & 5.93 & 4.70 & 4.87 & 4.97 & 4.66 \\
\hline $72 \ldots$ & 4.47 & 4.55 & 4.66 & 6.40 & 6.9 & 4.63 & 4.66 & 5.95 & 4.66 & 4.87 & 515 & 4.58 \\
\hline $81 \ldots$ & 4.51 & 4.53 & 4.59 & $\ldots$ & $\ldots$ & 4.53 & -... & $\ldots$ & 4.61 & 4.85 & - - - - & 4.55 \\
\hline $97 \ldots$ & 4.46 & 4.55 & 4.64 & - . - & $\ldots$ & 4.52 & ... & -..- & 4.64 & 4.87 & $\ldots$ & 4.61 \\
\hline
\end{tabular}

$$
\frac{\left[\mathrm{Al}^{+3}\right]\left[\mathrm{Si}(\mathrm{OH})_{4}\right]}{\left[\mathrm{H}^{+}\right]^{3}}=\mathrm{K} .
$$

From the following thermodynamic data, it is possible to evaluate this equilibrium constant for three forms of clay, ranging from relatively unstable synthetic halloysite to well-crystallized kaolinite.

\begin{tabular}{|c|c|c|}
\hline Species & $\Delta \mathrm{G}_{\mathrm{f}}^{\mathrm{o}}(\mathrm{kcal} / \mathrm{mole})$ & Reference \\
\hline - & -115.0 & Latimer (1952). \\
\hline $\mathrm{Si}(\mathrm{OH})_{4}$ & -312.7 & Polzer and Hem (1965). \\
\hline $\mathrm{H}_{2} \mathrm{O}$ & -56.69 & Latimer (1952). \\
\hline $\mathrm{Al}_{2} \mathrm{Si}_{2} \mathrm{O}_{5}(\mathrm{OH})_{4}:$ halloysite & -898.4 & Robie and Waldbaum (1968). \\
\hline Synthetic halloysite & $-897 \pm 1.0$ & $\begin{array}{l}\text { Hem, Roberson, Lind, and } \\
\text { Polzer (1973). }\end{array}$ \\
\hline $\mathrm{Al}_{2} \mathrm{Si}_{2} \mathrm{O}_{5}(\mathrm{OH})_{4}:$ kaolinite & -902.87 & Robie and Waldbaum (1968). \\
\hline Mineral & $\begin{array}{l}\Delta \mathrm{G}_{\text {reaction }} \\
\text { (kcallmole })\end{array}$ & $\mathrm{K}$ at $25^{\circ} \mathrm{C}$ for $\frac{[\mathrm{Al}+3][\mathrm{SirOH} / 4]}{\left[\mathrm{H}^{+}\right]^{3}}$ \\
\hline Halloysite & -6.84 & $10^{5.01}$ \\
\hline Synthetic halloysite & -7.54 & $10^{5.53}$ \\
\hline Kaolinite & -4.61 & $10^{3.38}$ \\
\hline
\end{tabular}


The following relation can be used to calculate the thermodynamic activity of $\mathrm{Al}^{+3}$ in solutions of 0.01 ionic strength when $\mathrm{pH}$ and total $\mathrm{Al}_{\mathrm{a}}$ are known:

$$
\left[\mathrm{Al}^{+3}\right]=\frac{\left(\mathrm{Al}_{\mathrm{a}}\right)}{2.34+\frac{1.47 \times 10^{-5}}{\left[\mathrm{H}^{+}\right]}+\frac{1.91 \times 10^{-10}}{\left[\mathrm{H}^{+}\right]^{2}}+\frac{7.6 \times 10^{-23}}{\left[\mathrm{H}^{+}\right]^{4}}} .
$$

The quantity $\left(\mathrm{Al}_{\mathrm{a}}\right)$ is the analytically determined value in moles per litre. Calculated values for $\left[\mathrm{Al}^{+3}\right]$ after approximately 100 days of aging are given in table 5 . The activities of silicic acid $\left(\mathrm{Si}(\mathrm{OH})_{4}\right)$ in table 5 were calculated from the determined silica, assuming that this uncharged species has an activity coefficient of unity. The activity of $\mathrm{OH}^{-}$was calculated from the measured $\mathrm{pH}$, using $10^{-14.00}$ as the ion activity product for water.

The data in table 5 all represent the analysis made at the maximum aging time that gave useful information for calculating equilibrium solubility. Generally this was near 100 days. For some of the solutions, however, especially those having the larger amounts of silica, the concentration of $\mathrm{Al}_{\mathrm{a}}$ dropped below the detection linit before that time. For these solutions a somewhat shorter aging period was used, for which a specific value for $\mathrm{Al}_{\mathrm{a}}$ was available. The cation exchange capacity values in table 5 represent concentrations in equivalents per litre, calculated from the weights of solids added and their measured cation exchange capacity per unit weight.

From inspection of the plot of $\mathrm{pAl}+\mathrm{pSi}(\mathrm{OH})_{4}$ versus $\mathrm{pH}$ (fig. 5), it is apparent that several solutions from each group are surersaturated or near saturation with respect to synthetic halloysite. Generally those solutions containing 0.1 and $0.2 \mathrm{~g}$ Wyoming bentonite and 0.5 and 2.0 $\mathrm{g}$ kaolinite are those whose aluminum solubility may b? controlled by silicate equilibria. The aluminum concentrations in these solutions may therefore be controlled by precipitation of aluminosilicate, although it is not possible to verify the existence of this kind of solid material in the presence of the added mineral materic 1 . A few of the solutions contained more than the $9 \mathrm{mg} / \mathrm{l} \mathrm{SiO}{ }_{2}$ specified by Hem, Roberson, Lind, and Polzer (1973) as the upper limit for dissolved silica in solutions in which microcrystalline gibbsite vas ultimately identified. It appears reasonable and proper to leave data from these solutions out of consideration in evaluating the behavicr of aluminum in systems where dissolved silica is absent. It has been noted that Wyoming bentonite and volcanic ash, both having beer found to have high points of zero charge ( 8.1 and 8.4 , respectivel:r), could have increased the $\mathrm{pH}$ by direct sorption of $\mathrm{H}^{+}$. Kaolinite, having a low 
TABLE 5.-Aluminosilicate and gibbsite ion activity products and related data for solutions $D, F, G$, and $H$ after $2-3$ months aging

\begin{tabular}{|c|c|c|c|c|c|c|c|c|c|}
\hline Solution & 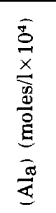 & 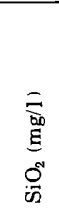 & $\begin{array}{l}5 \\
+ \\
\frac{2}{2}\end{array}$ & 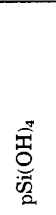 & $\begin{array}{l}\text { I } \\
\text { 口 }\end{array}$ & 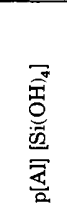 & 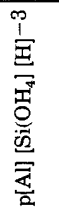 & 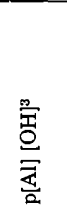 & 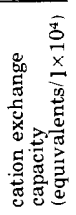 \\
\hline 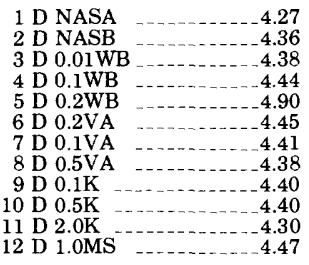 & $\begin{array}{r}1.99 \\
1.93 \\
1.88 \\
1.04 \\
.09 \\
1.91 \\
1.95 \\
.94 \\
1.80 \\
1.76 \\
1.44 \\
1.67\end{array}$ & $\begin{array}{r}0.0 \\
.0 \\
.9 \\
1.6 \\
1.4 \\
.7 \\
.8 \\
5.3 \\
1.2 \\
3.4 \\
13.6 \\
.6\end{array}$ & $\begin{array}{l}4.13 \\
4.16 \\
4.17 \\
4.44 \\
5.72 \\
4.18 \\
4.16 \\
4.47 \\
4.19 \\
4.21 \\
4.27 \\
4.25\end{array}$ & $\begin{array}{l}4.82 \\
4.57 \\
5.18 \\
4.93 \\
4.88 \\
4.05 \\
4.70 \\
4.25 \\
3.65 \\
5.00\end{array}$ & $\begin{array}{l}9.73 \\
9.64 \\
9.62 \\
9.56 \\
9.10 \\
9.55 \\
9.59 \\
9.62 \\
9.60 \\
9.60 \\
9.70 \\
9.53\end{array}$ & $\begin{array}{r}8.99 \\
9.01 \\
10.90 \\
9.11 \\
9.04 \\
8.52 \\
8.89 \\
8.46 \\
7.92 \\
9.25\end{array}$ & $\begin{array}{l}4.15 \\
4.31 \\
3.80 \\
4.24 \\
4.19 \\
4.62 \\
4.31 \\
4.74 \\
4.98 \\
4.16\end{array}$ & $\begin{array}{l}33.32 \\
33.08 \\
33.03 \\
33.12 \\
33.02 \\
32.82 \\
32.93 \\
33.33 \\
32.99 \\
33.01 \\
33.37 \\
32.84\end{array}$ & $\begin{array}{l}0.369 \\
3.69 \\
7.38 \\
.211 \\
1.06 \\
5.28 \\
.276 \\
1.38 \\
5.52 \\
.264\end{array}$ \\
\hline 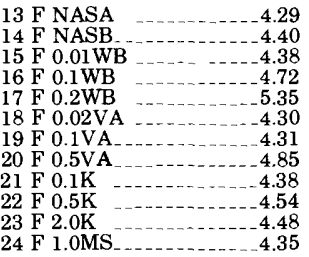 & $\begin{array}{r}1.21 \\
1.20 \\
1.14 \\
.20 \\
.24 \\
1.25 \\
1.06 \\
.19 \\
1.26 \\
1.13 \\
.80 \\
1.19\end{array}$ & $\begin{array}{r}.0 \\
.0 \\
.0 \\
.5 \\
2.5 \\
.4 \\
1.4 \\
1.0 \\
1.4 \\
3.7 \\
13.4 \\
.3\end{array}$ & $\begin{array}{l}4.35 \\
4.37 \\
4.39 \\
5.26 \\
5.81 \\
4.34 \\
4.41 \\
5.36 \\
4.35 \\
4.44 \\
4.57 \\
4.37\end{array}$ & $\begin{array}{l}--\cdots \\
-\cdots \\
5.08 \\
4.38 \\
5.18 \\
4.63 \\
4.78 \\
4.63 \\
4.21 \\
3.65 \\
5.30\end{array}$ & $\begin{array}{l}9.71 \\
9.60 \\
9.62 \\
9.28 \\
8.65 \\
9.70 \\
9.69 \\
9.15 \\
9.62 \\
9.46 \\
9.52 \\
9.65\end{array}$ & $\begin{array}{r} \\
--.- \\
10.34 \\
10.19 \\
9.52 \\
9.04 \\
10.14 \\
8.98 \\
8.65 \\
8.22 \\
9.67\end{array}$ & $\begin{array}{l}-.- \\
3.82 \\
5.86 \\
3.38 \\
3.89 \\
4.41 \\
4.16 \\
4.97 \\
5.22 \\
3.38\end{array}$ & $\begin{array}{l}33.48 \\
33.47 \\
33.25 \\
33.10 \\
31.76 \\
33.44 \\
33.48 \\
32.81 \\
33.21 \\
32.82 \\
33.13 \\
33.32\end{array}$ & $\begin{array}{c} \\
.369 \\
3.69 \\
7.38 \\
.211 \\
1.06 \\
5.28 \\
.276 \\
1.38 \\
5.52 \\
.264\end{array}$ \\
\hline 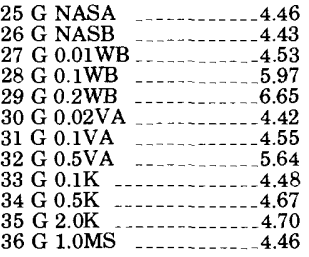 & $\begin{array}{l}.70 \\
.98 \\
.57 \\
.09 \\
.18 \\
.61 \\
.55 \\
.00 \\
.66 \\
.52 \\
.27 \\
.43\end{array}$ & $\begin{array}{r}.0 \\
.0 \\
.0 \\
.9 \\
.9 \\
.6 \\
1.2 \\
3.9 \\
1.4 \\
2.4 \\
7.7 \\
.0\end{array}$ & $\begin{array}{l}4.62 \\
4.47 \\
4.73 \\
7.43 \\
9.28 \\
4.67 \\
4.75 \\
4.65 \\
4.82 \\
5.12 \\
4.83\end{array}$ & $\begin{array}{l}---- \\
--- \\
4.82 \\
4.82 \\
5.00 \\
4.70 \\
4.19 \\
4.63 \\
4.40 \\
3.89\end{array}$ & $\begin{array}{l}9.54 \\
9.57 \\
9.47 \\
8.03 \\
7.35 \\
9.58 \\
9.45 \\
8.36 \\
9.52 \\
9.33 \\
9.30 \\
9.54\end{array}$ & $\begin{array}{r}--- \\
--- \\
12.25 \\
14.10 \\
9.67 \\
9.45 \\
\overline{9} .28 \\
9.22 \\
9.01 \\
-\end{array}$ & $\begin{array}{l}---- \\
---- \\
\overline{5} .66 \\
5.85 \\
3.59 \\
4.20 \\
- \\
3.92 \\
4.79 \\
5.09 \\
----\end{array}$ & $\begin{array}{l}33.24 \\
33.18 \\
33.14 \\
31.52 \\
31.33 \\
33.41 \\
33.10 \\
33.21 \\
32.81 \\
33.02 \\
33.45\end{array}$ & $\begin{array}{c}- \\
0.369 \\
3.69 \\
7.38 \\
.211 \\
1.06 \\
5.28 \\
.276 \\
1.38 \\
5.52 \\
.264\end{array}$ \\
\hline 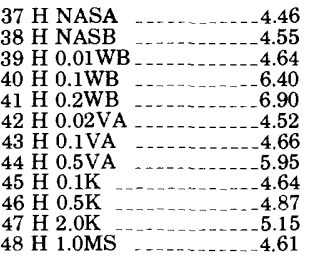 & $\begin{array}{l}.48 \\
.42 \\
.34 \\
.11 \\
.23 \\
.46 \\
.27 \\
.09 \\
.31 \\
.30 \\
.05 \\
.40\end{array}$ & $\begin{array}{r}.0 \\
.0 \\
.4 \\
.7 \\
1.2 \\
.4 \\
1.2 \\
4.2 \\
1.7 \\
3.3 \\
9.7 \\
.3\end{array}$ & $\begin{array}{r}4.79 \\
4.87 \\
4.99 \\
8.59 \\
10.14 \\
4.82 \\
5.10 \\
7.38 \\
5.03 \\
5.17 \\
6.22 \\
4.91\end{array}$ & $\begin{array}{l}-.18 \\
5.18 \\
4.70 \\
5.18 \\
4.70 \\
4.16 \\
4.55 \\
4.26 \\
3.79 \\
5.30\end{array}$ & $\begin{array}{l}9.54 \\
9.45 \\
9.36 \\
7.60 \\
7.10 \\
9.48 \\
9.34 \\
8.05 \\
9.36 \\
9.13 \\
8.85 \\
9.39\end{array}$ & $\begin{array}{r} \\
10.17 \\
13.52 \\
14.84 \\
10.00 \\
9.80 \\
11.54 \\
9.58 \\
9.43 \\
10.01 \\
10.21\end{array}$ & $\begin{array}{l}\cdots .75 \\
5.68 \\
5.86 \\
3.56 \\
4.18 \\
6.31 \\
4.34 \\
5.18 \\
5.44 \\
3.62\end{array}$ & $\begin{array}{l}33.41 \\
33.22 \\
33.07 \\
31.39 \\
31.44 \\
33.26 \\
33.12 \\
31.53 \\
33.11 \\
32.56 \\
32.77 \\
33.08\end{array}$ & $\begin{array}{c}-- \\
.369 \\
3.69 \\
7.38 \\
.211 \\
1.06 \\
5.28 \\
.276 \\
1.38 \\
5.52 \\
.264\end{array}$ \\
\hline
\end{tabular}

isoelectric point of 4.3 , should have had little effect on the pH's of the solutions containing it.

As shown in the section on $\mathrm{Al}_{\mathrm{b}}$ measurements, those solutions that are here shown to be supersaturated with respect to aluminum silicate showed very rapid decreases in $\mathrm{Al}_{\mathrm{b}}$ concentrations. Within 2-5 days $\mathrm{Al}_{\mathrm{b}}$ was below the approximately $10^{-6}$ molar detection limit, with only two or three points to trace the rate of polymerization. As to whether this sudden decrease is really due to surface catalyzed polymerization to form gibbsite or to reaction of the aluminum hydroxide polymer 


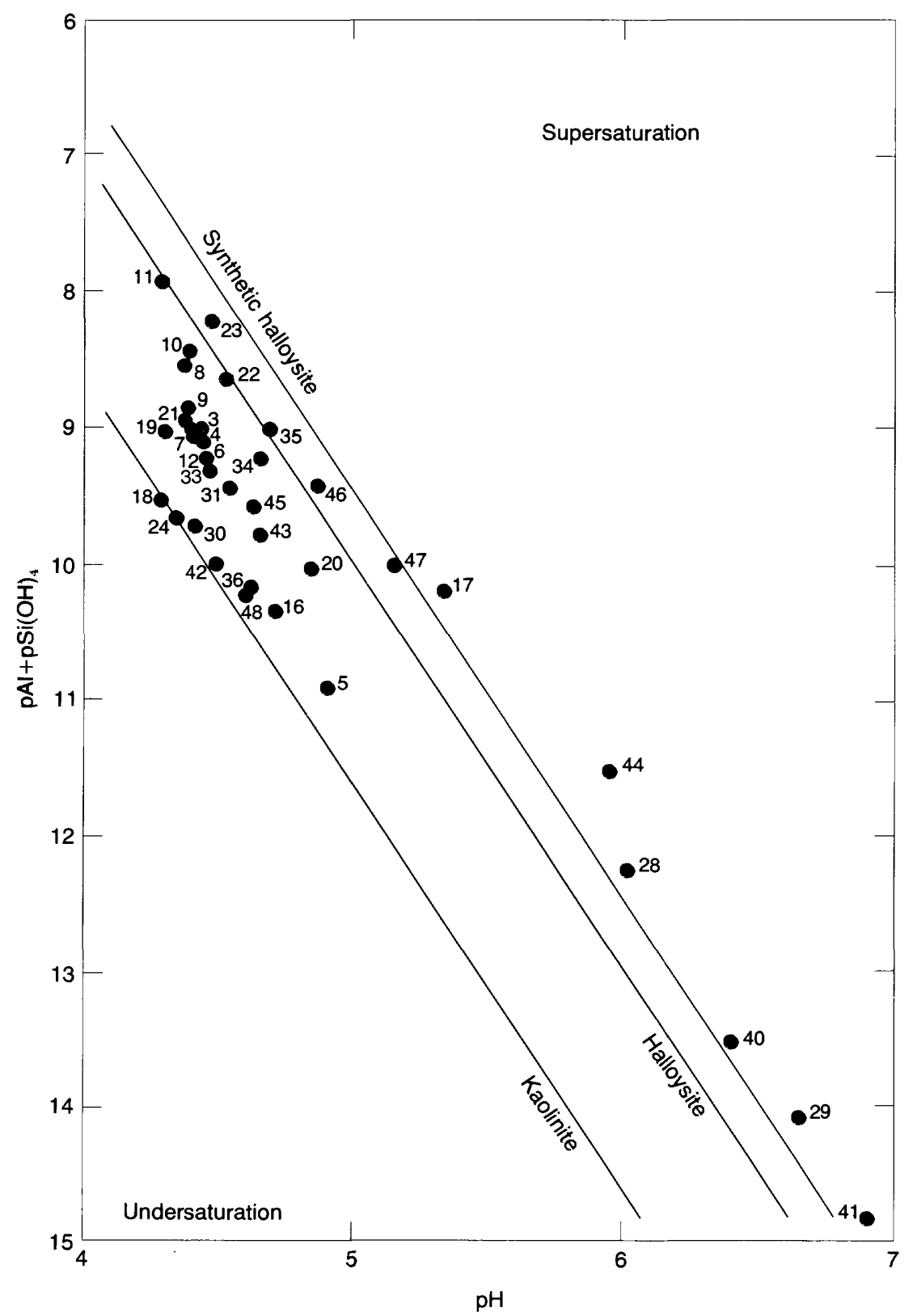

FIgURE 5.-Clay-mineral solubilities compared with compositions of experimental solutions. Numbers beside points correspond to solutions likewise numbered in table 5 . 
with silica released from the surfaces, we can say with some certainty that in the cases mentioned, the clay mineral surfaces have caused chemical interferences and have introduced factors other than those of an inert surface catalyzing aluminum hydroxide polymerizat in. Because of this effect, those solutions calculated to be supersaturated or near saturation with respect to aluminosilicates will not be further studied for comparison of polymerization rates and rate constants. However, since the effects of aluminosilicate solubility most likely become dominant only after several weeks aging and any adsorption of $\mathrm{Al}_{\mathrm{a}}$ or $\mathrm{Al}_{\mathrm{b}}$ by the surface took place after only a few hours, as later shown, the adsorption of $\mathrm{AI}_{a}$ and $\mathrm{Al}_{b}$ in these solutions can be considered.

\section{GIBBSITE EQUILIBRIUM SOLUBILITY}

The solubility constant for gibbsite given by Smith and Hem (1972) for the expression $K=\left[\mathrm{Al}^{+3}\right]\left[\mathrm{H}^{+}\right]^{-3}$ is $10^{8.22}$. This represents the behavior of well-crystallized material produced by long agir $\mathrm{g}$ (more than 2 years in some instances). If written as a conventional solubility product,

$$
K_{s 0}=\left[\mathrm{Al}^{+3}\right]\left[\mathrm{OH}^{-}\right]^{3},
$$

this becomes $10^{-33.78}$. Hem and Roberson (1967) reported a value for this solubility product of $10^{-32.65}$, representing microcrystalline gibbsite. Their solid was aged for a shorter time.

Values are given in table 5 for $K_{s 0}$ for solutions D, F, G, and $\mathrm{H}$ after $2-3$ months of aging. The activity products for these systems lie between the $K_{s 0}$ values for microcrystalline gibbsite and the more stable final form. This is illustrated in another way by plotting $-\operatorname{lng}[\mathrm{Ai}+3]$ versus $\mathrm{pH}$ in figure 6.

A few points would have plotted substantially to the right (supersaturation) of even the microcrystalline gibbsite line. These are for solutions that gave evidence of attack on the surfaces and cannot be considered significant when treated in this simplified equilibrium model.

In general the positions of the points where effects of silica are absent are about what would be predicted for the length of aging, using the criteria of Smith and Hem (1972). As the solutions age, it would be expected the points would move toward the left hand gibbsite solubility limit.

Although the data in tables 1-5 show a considerable loss in $\mathrm{Al}_{\mathrm{a}}$ occurred when the surfaces were present, the behavior of $\mathrm{Al}_{\mathrm{a}}$ generally seems to fit that of a gibbsite equilibrium system. A more detailed examination of the data is obviously required before the behavior of $\mathrm{Al}_{\mathrm{a}}$ can be fully explained. 


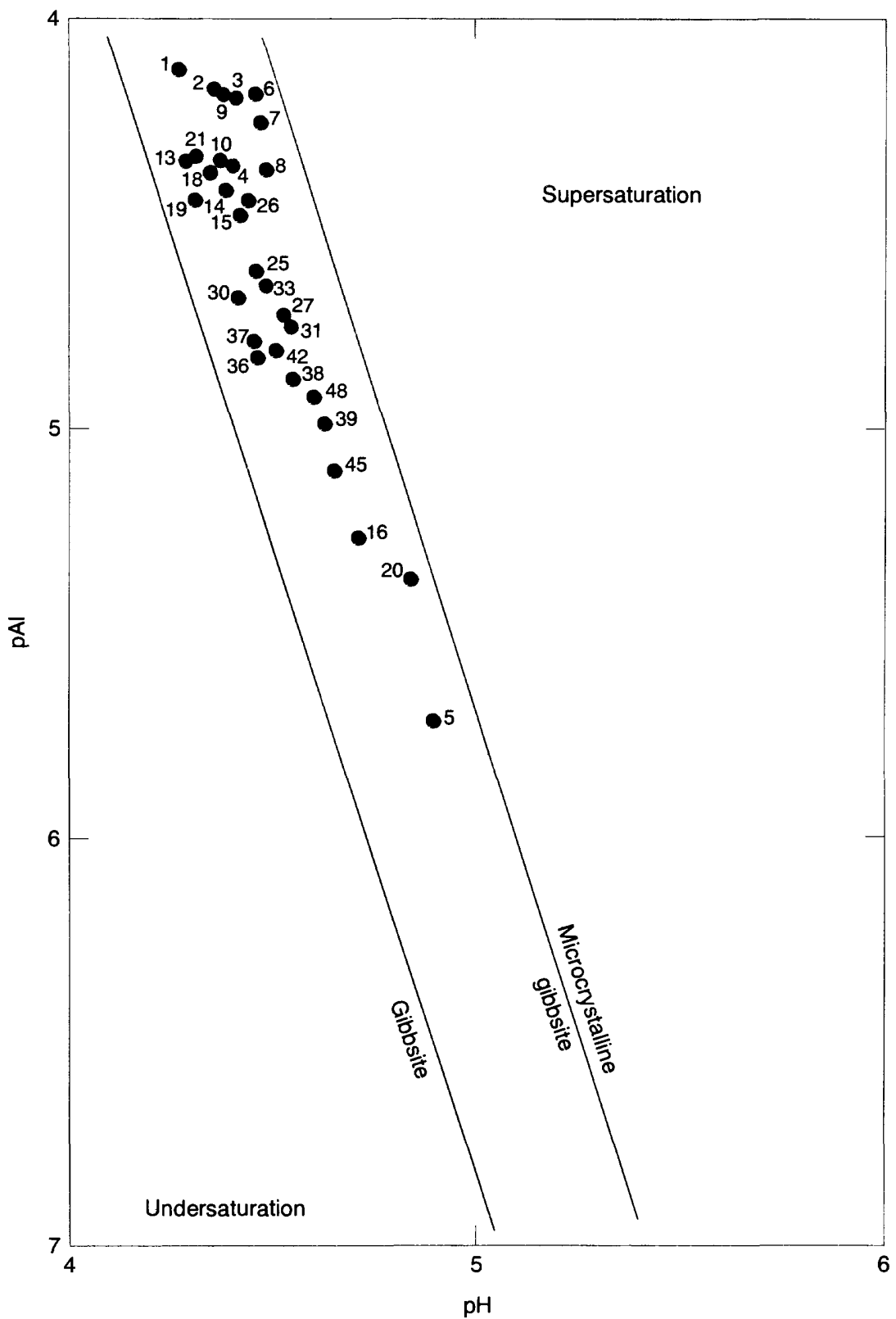

F1GURE 6.-Gibbsite solubilities compared with compositions of experimental solutions. Numbers beside points correspond to solutions likewise numbered in table 5 . 
Smith and Hem (1972) observed a substantial decrease in pH during aging of their solutions when the ratio of bound hydroxide to aluminum was 0.94 or greater. This decrease was attributed to the process of polymerization of $\mathrm{Al}_{\mathrm{b}}$, and they were able to demor strate a stoichiometric relationship. In solutions where polymerization did not occur, the $\mathrm{pH}$ remained essentially constant. The approach of the solutions to equilibrium with respect to gibbsite occurred mainly through the decreasing $\mathrm{pH}$. It is of interest to compare $\mathrm{pH}$ changes in the solutions having mineral surfaces with corresponding ones that had no mineral surfaces.

Although the solutions D, F, G, and H prepared for this work were made by mixing amounts of stock solutions that were identical to those used by Smith and Hem (1972), the composition of the final mixtures differed somewhat from those of the earlier study. The stock solutions were mixed slowly with the aim of maximizing the $\mathrm{Al}_{\mathrm{b}}$ concentration. As a result the $\mathrm{Al}_{\mathrm{b}}$ content was substantially higher, $\mathrm{Al}_{\mathrm{a}}$ somewhat lower, and $\mathrm{pH}$ a little lower than in corresponding solutiors of the earlier study. As noted by Smith and Hem (1972), slow addition of the basic solution during mixing tends to produce a solution farther from equilibrium than rapid addition does, in that slow addition $\mathrm{F}^{\text {romotes }}$ the formation of $\mathrm{Al}_{b}$, a nonequilibrium species.

The solutions that did not contain added mineral surfaces did generally follow the previously observed patterns after mixing. The $\mathrm{pH}$ usually decreased in these solutions by an amount similar to that observed by Smith and Hem in the first 100 days that their solutions were aged.

The values of $\mathrm{pH}$ observed in the final analysis of our aging solutions, made after about 100 days of aging, are given in table 5 . It is evident that the solutions to which the larger amounts of solid minerals were added generally had a higher $\mathrm{pH}$ after aging than the other solutions. Furthermore, the observations of $\mathrm{pH}$ made durirg aging (tables 1-4) show that many of these solutions had only small changes in $\mathrm{pH}$ after the initial observation, made just after time zero when the solutions were first prepared and mineral surfaces were adc'ed.

It seems obvious that the mineral surfaces influenced th $\odot$ aging process in some way; however, the systems involved are more complex than those discussed in Smith and Hem (1972), and several possible processes may have been involved. As previously stated, actual attack and partial dissolution of the surfaces probably occurred in some of the solutions, resulting in losses of $\mathrm{H}^{+}$by reaction with surficial material. 
Also, reactions involving aluminosilicate precipitation could occur. The final equilibrium condition might thus be shifted avay from one involving only gibbsite. Another possible effect is the adsorption of cations by the mineral surface. Direct sorption of $\mathrm{H}^{+}$would, of course, increase the $\mathrm{pH}$, and preferential sorption of any of the various forms of aqueous aluminum also would have indirect effects on $\mathrm{pH}$. It is noteworthy that the solutions with highest $\mathrm{pH}$ generally are the ones in each group with large exchange capacity associated with bentonite and volcanic ash; the solutions containing the largest amount of kaolinite show a less prominent $\mathrm{pH}$ increase. In any event, these are only three of possibly several causes for differences observed in these solutions.

The effects of adsorption and cation exchange on aluminum species concentrations will be considered later in this paper.

\section{CHANGES IN $\mathrm{Al}_{\mathrm{d}}$ AND $\mathrm{Al}_{\mathrm{b}}$ CONCENTRATION DURING AGING}

As mentioned earlier, measurements of aqueous $\mathrm{Al}_{\mathrm{a}}$ c nd $\mathrm{Al}_{\mathrm{b}}$ were made periodically on each group of solutions. In the analytical procedure where no surfaces have been added to the solution, the amount of $\mathrm{Al}_{\mathrm{c}}$ is calculated by difference, since it is assumed that $\Sigma \mathrm{Al}=\mathrm{Al}_{\mathrm{a}}+\mathrm{Al}_{\mathrm{b}}+\mathrm{Al}_{\mathrm{c}}$.

It should be noted that the $\mathrm{Al}_{\mathrm{a}}$ species concentration is a directly determined value. Differences between the $\mathrm{Al}_{\mathrm{a}}$ content of the blank solution and the solutions containing surfaces, therefore, are valid measurements of the $\mathrm{Al}_{\mathrm{a}}$ losses (or $\Delta \mathrm{Al}_{\mathrm{a}}$ ). The results may be used to evaluate the nature and rates of processes at the mineral surfaces that are responsible for the loss of aqueous $\mathrm{Al}_{\mathrm{a}}$.

Similarly, aqueous $\mathrm{Al}_{\mathrm{b}}$ can be measured in a somewhat less direct way, and $\Delta \mathrm{Al}_{\mathrm{b}}$ can be computed by comparing values for the blank solution with the comparable values for solutions containing surfaces. If there are significant differences in behavior of $\mathrm{Al}_{\mathrm{a}}$ and $\mathrm{Al}_{\mathrm{b}}$, the experimental results should permit at least a generalizec' evaluation.

The third form of aqueous aluminum evaluated by Smith and Hem (1972), $\mathrm{Al}_{c}$, presents more difficult problems. In the absence of surfaces, it can perhaps be assumed, as was done previously, that the difference between the total initial aluminum concentration and the measured values of $\mathrm{Al}_{\mathrm{a}}+\mathrm{Al}_{\mathrm{b}}$ at time $t$ represents the concentration of $\mathrm{Al}_{\mathrm{c}}$. This material is in the colloidal size range, or near it, and cannot be directly measured, even where solid mineral surfaces are not present, although qualitative evidence of its presence is readily obtainable. When surfaces are present, there is still another species of aluminum to be considered, the adsorbed aluminum, which cannot be readily determined separately from the $\mathrm{Al}_{\mathrm{c}}$. The total of both can be calculated as the difference between $\mathrm{Al}_{\mathrm{a}}+\mathrm{Al}_{\mathrm{b}}$ and the total initial 
aluminum for any of the solutions at a specific time after the surface was added.

Changes in the concentration of $\mathrm{Al}_{\mathrm{a}}$ between 1 and $100 \mathrm{c}^{\prime}$ ays were observed in many of these solutions, although the changes generally were small. Smith and Hem (1972) reported no change of significance occurred in $\mathrm{Al}_{\mathrm{a}}$ in their solutions during aging.

It does not seem possible to attribute much significanse to the changes in $\mathrm{Al}_{\mathrm{a}}$ that can be seen in tables $1-4$. Some solutions show small gains in $\mathrm{Al}_{\mathrm{a}}$, and others show losses. There is no clear-cut relationship to amount or kind of solid present. Some depolymerization of aqueous $\mathrm{Al}_{\mathrm{b}}$ or desorption of aluminum species might have occurred as the $\mathrm{pH}$ changed during aging and polymerization.

The $\mathrm{Al}_{\mathrm{b}}$ contents show a much more consistent trend and, as in the solutions containing no added surface, the trend was downward throughout the aging period.

Smith and Hem (1972) observed a first-order disappearance of $\mathrm{Al}_{\mathrm{b}}$ over a considerable part of the aging period, and the half-time of this process appears from their data to have been about 20 days. However, the $\mathrm{Al}_{\mathrm{b}}$ content of most of their solutions changed much mcre slowly during the first 20 days than during the next 100 days, where the first-order rate mechanism was well defined.

Values of $\log \mathrm{Al}_{b}$ concentration and time in days were taken from tables 1-4 and plotted. The results for solutions in the F, G, and $\mathrm{H}$ series generally correspond to those obtained by Smith and Hem, in that the data generally show a first-order rate mechanism and the half-time of the process is about 20 days. Most of the solutions in the D series lost very little $\mathrm{Al}_{\mathrm{b}}$ during 103 days of aging. The initial $\mathrm{pH}$ of these D solutions was in a range where Smith and Hem's result shows little polymerization took place during an equivalent period of time.

Typical graphs of $\log \mathrm{Al}_{\mathbf{b}}$ concentration versus time in days are given as figures 7 and 8 . The polymerization rate is evidently not significantly influenced by the presence of mineral surfaces of the types used here. However, the surfaces do have a readily discernible effect in the early part of the aging process, because the amount of $\mathrm{Al}_{\mathrm{b}}$ present at the time of the first measurement can be seen in tables 1-4 to differ from one solution to another and the lower values represent solutions to which mineral surfaces had been added. A closer study of the effects produced immediately upon addition of the solid materials seems, therefore, to be required.

\section{CONCENTRATION OF $\mathrm{Al}_{\mathrm{b}}$ AT TIME ZERO}

From examining first-order rate curves drawn across the plots of $-\log \left(\mathrm{Al}_{\mathrm{b}}\right)$ versus age time, it can be observed that the value for $-\log$ $\left(\mathrm{Al}_{\mathrm{b}}\right)$ at zero age time, that is immediately after addition of mineral 


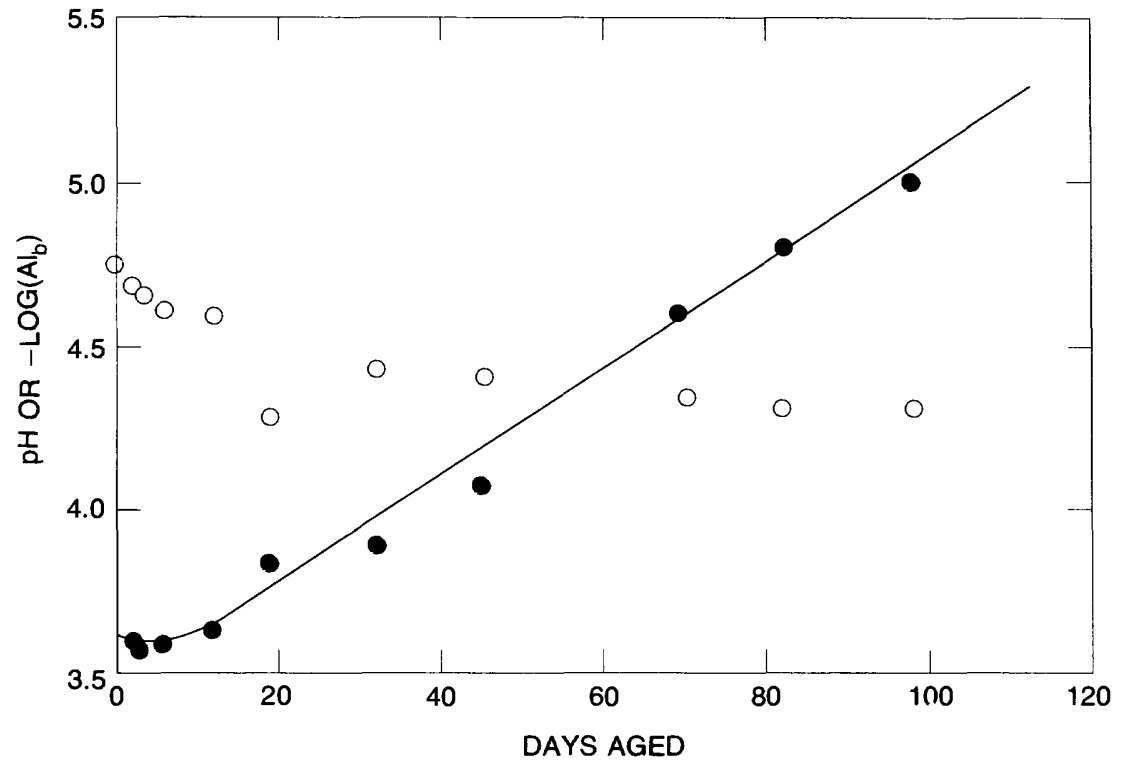

FiguRE 7.-First-order disappearance of $\mathrm{Al}_{\mathrm{b}}$, $\mathrm{pH}$ (circles) and $-\log \left(\mathrm{Al}_{\mathrm{b}}\right)(\operatorname{dots})$ as a function of aging time for representative solution $\mathrm{F} 0.02 \mathrm{VA}$.

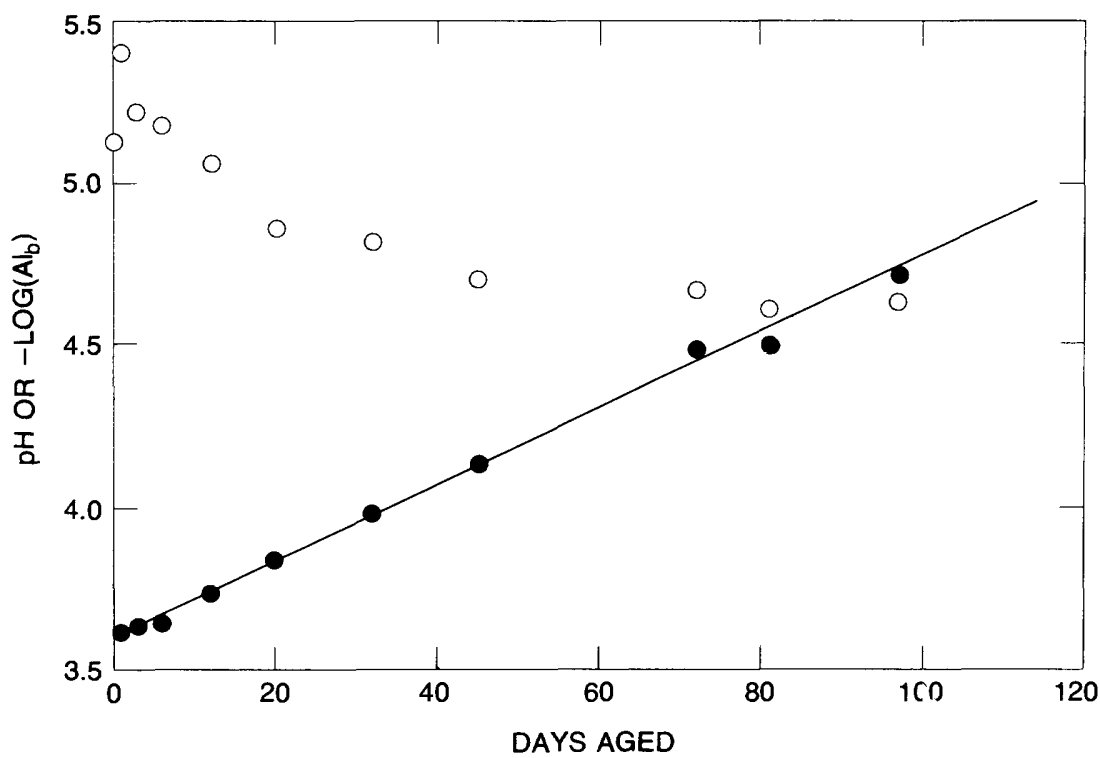

Figure 8.-First-order disappearance of $\mathrm{Al}_{\mathrm{b}}, \mathrm{pH}$ (circles) and - $\log \left(\mathrm{Al}_{\mathrm{b}}\right)$ (dots) as a function of aging time for representative solution $\mathrm{H} 0.1 \mathrm{~K}$. 
surfaces, varies widely within a given set of solutions. This initial value appears to be dependent on the amount and type of surface added.

Within each set of solutions, those with no added surface contained greater $\mathrm{Al}_{\mathrm{b}}$ concentrations than any of the ones to which mineral surfaces were added. This indicates that on addition of surface, a substantial amount of $\mathrm{Al}_{b}$ was lost. Because the first-order polymerization rates are too slow to account for such rapid polymerization during the 1 or 2 days before the first analysis, this loss of $\mathrm{Al}_{\mathrm{b}}$ is probably attributable to adsorption by the surface. If the difference in initial values of $-\log \left(\mathrm{Al}_{\mathbf{b}}\right)$ is attributable to adsorption by the surface, the amount adsorbed, in terms of concentration, may be calculated by subtracting the $\mathrm{Al}_{\mathrm{b}}$ concentration in question from the $\mathrm{Al}_{\mathrm{b}}$ cor centration of the solution to which no surface was added. This quantity is shown in the last column of table 6 .

From data in table 6, it appears that the greatest losses of $\mathrm{Al}_{\mathrm{b}}$ occurred in solutions containing the greatest area of solid surface. The relationship between $\mathrm{Al}_{b}$ loss and surface area is demonstrated in figures 9-12. Although some of the points deviate from the regression lines, the results suggest that differences in the properties of the mineral surfaces used in this study are of relatively small significance and loss of $\mathrm{Al}_{\mathrm{b}}$ is primarily related to area of surface available.

Smith and Hem (1972, p. 32) attributed the decline in $\mathrm{pH}$ th at they observed in their solutions during aging to the production of $\mathrm{Al}_{\mathrm{c}}$ by polymerization of $\mathrm{Al}_{\mathrm{b}}$. A somewhat similar decline can be seen in the $\mathrm{pH}$ of solutions without the added surface listed in tables 1- 4 of this paper. However, the solutions to which mineral surfaces wers added do not show $\mathrm{pH}$ changes that correlate with $\mathrm{Al}_{\mathrm{b}}$ loss. From data in tables $1-4$, it is evident that most solutions where $\mathrm{Al}_{\mathrm{b}}$ loss oncurred immediately after the surfaces were added did not decrease in $\mathrm{pH}$ to the extent one might expect had the $\mathrm{Al}_{\mathrm{b}}$ polymerization gone to completion at the surface. It would seem, therefore, that the los ${ }^{\circ}$ of $\mathrm{Al}_{\mathrm{b}}$ represents an adsorption rather than catalysis of the polymerization process.

It is possible the surfaces also adsorbed some $\mathrm{H}^{+}$ard thus influenced the $\mathrm{pH}$. This effect cannot be completely evaluated, but it is believed to be minor. The solutions with surfaces in which $\mathrm{Al}_{\mathrm{b}}$ loss continued during aging decreased in $\mathrm{pH}$, suggesting that the effect of the polymerization reaction can be observed, when it occurs, even if the surfaces are present.

SIGNIFICANCE OF CATION EXCHANGE CAPACITY

The CEC (cation exchange capacity) of added surfaces is sl nwn in 
TABLE 6.-Short-term losses of $\mathrm{Al}_{\mathrm{b}}$ in solutions $D, F, G$, and $H$ [Dash leaders indicate $\mathrm{Al}_{\mathrm{b}}$ loss so rapid that $\mathrm{Al}_{\mathrm{b}}$ at time zero could not be confidently extrapolated]

\begin{tabular}{|c|c|c|c|c|}
\hline \multirow{2}{*}{$\begin{array}{l}\text { Solution } \\
\text { D NASA }\end{array}$} & $\begin{array}{l}\text { Added surface area } \\
\left(\mathrm{m}^{2} / \mathrm{l}\right)\end{array}$ & \multicolumn{2}{|c|}{$\begin{array}{c}\text { Extrapolated } \\
-\log \text { Alb }\end{array}$} & $\begin{array}{c}\text { Cor centration differences } \\
\left(\text { moles } / \text { litre } \times 10^{4}\right)\end{array}$ \\
\hline & --------- & $3.70\}$ & mean, & \\
\hline D NASB & - & $3.67\}$ & 3.68 & 0.00 \\
\hline D $0.01 \mathrm{WB}$ & 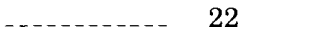 & 3.94 & & .94 \\
\hline D $0.1 \mathrm{WB}$ & 220 & 4.90 & & 1.96 \\
\hline D $0.2 W B$ & $\ldots$ & & & -- - \\
\hline D $0.02 \mathrm{VA}$ & - - - - n- - - - - & 3.72 & & .18 \\
\hline D $0.1 \mathrm{VA}$ & - - $-\ldots+\ldots-\ldots$ & 4.07 & & 1.24 \\
\hline D $0.5 \mathrm{VA}$ & 220 & & & $-\ldots$ \\
\hline $\mathrm{D} 0.1 \mathrm{~K}$ & 6.0 & 3.76 & & .35 \\
\hline D $0.5 \mathrm{~K}$ & - & 3.89 & & .80 \\
\hline D $2.0 \mathrm{~K}$ & 120 & 4.49 & & 1.77 \\
\hline D 1.0MS & ---------- & 3.66 & & .00 \\
\hline F NASA & --------- & $3.50\}$ & mean, & .00 \\
\hline F NASB & - & $3.50\}$ & 3.50 & .00 \\
\hline F $0.01 \mathrm{WB}$ & $--\ldots-\ldots-\ldots-n--$ & 3.75 & & 1.38 \\
\hline F $0.1 \mathrm{WB}$ & 220 & ---- & & $-\cdots$ \\
\hline F $0.2 \mathrm{WB}$ & 440 & & & ---- \\
\hline $\mathrm{F} 0.02 \mathrm{VA}$ & ----------- & 3.65 & & .92 \\
\hline F $0.1 \mathrm{VA}$ & 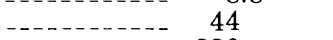 & 3.78 & & 1.50 \\
\hline F $0.5 \mathrm{VA}$ & 220 & & & ---- \\
\hline $\mathrm{F} 0.1 \mathrm{~K}$ & 6.0 & 3.65 & & .92 \\
\hline $\mathrm{F} 0.5 \mathrm{~K}$ & $-1--1---$ & 3.83 & & 1.68 \\
\hline $\mathrm{F} 2.0 \mathrm{~K}$ & 120 & 4.60 & & 2.91 \\
\hline F 1.0MS & ----------- & 3.57 & & .47 \\
\hline G NASA & $---\infty-1-n-n$ & $3.53\}$ & mean, & 00 \\
\hline G NASB & --- - - & $3.55\}$ & 3.54 & .00 \\
\hline G $0.01 \mathrm{WB}$ & 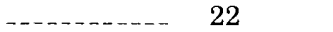 & 3.67 & & .75 \\
\hline G $0.1 \mathrm{WB}$ & 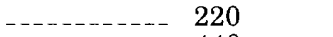 & 6.08 & & 2.88 \\
\hline G $0.2 W B$ & 440 & & & --- \\
\hline G $0.02 \mathrm{VA}$ & -n--n-n-n- & 3.58 & & .25 \\
\hline G $0.1 \mathrm{VA}$ & - n- & 3.72 & & .98 \\
\hline G $0.5 \mathrm{VA}$ & 220 & 3.72 & & .98 \\
\hline $\mathrm{G} 0.1 \mathrm{~K}$ & - n- & 3.58 & & .25 \\
\hline $\mathrm{G} 0.5 \mathrm{~K}$ & - n-n- & 3.70 & & .89 \\
\hline $\mathrm{G} 2.0 \mathrm{~K}$ & 120 & 4.3 & & 2.38 \\
\hline G $1.0 \mathrm{MS}$ & ------------ & 3.56 & & .13 \\
\hline H NASA & --n-n-n-n-- & $3.58\}$ & mean, & 00 \\
\hline H NASB & - - - - - & $3.58\}$ & 3.58 & \\
\hline $\mathrm{H} 0.01 \mathrm{WB}$ & 22 & 3.69 & & .59 \\
\hline H 0.1 WB & 220 & 4.65 & & 2.41 \\
\hline $\mathrm{H} 0.2 \mathrm{WB}$ & 440 & & & $\ldots-\ldots$ \\
\hline $\mathrm{H} 0.02 \mathrm{VA}$ & - n....... & 3.65 & & .39 \\
\hline $\mathrm{H} 0.1 \mathrm{VA}$ & - & 3.78 & & .97 \\
\hline $\mathrm{H} 0.5 \mathrm{VA}$ & 220 & 4.63 & & 2.40 \\
\hline $\mathrm{H}_{0.1 \mathrm{~K}}-$ & (n-1 & 3.63 & & .29 \\
\hline $\mathrm{H} 0.5 \mathrm{~K}$ & - & 3.85 & & 1.22 \\
\hline $\mathrm{H} 2.0 \mathrm{~K}$ & 120 & 5.0 & & 2.53 \\
\hline $\mathrm{H}$ 1.0MS & $\ldots-\ldots-\ldots-\ldots$ & 3.60 & & .12 \\
\hline
\end{tabular}

table 5 for each solution. The exchange positions on the surfaces present would, of course, be occupied at all times by cations taken up from the associated solution. Moreover, the nature of tho ions so held and the proportions of species in the exchange positions would be functions of the solution composition as well as of properties of the exchange sites. 


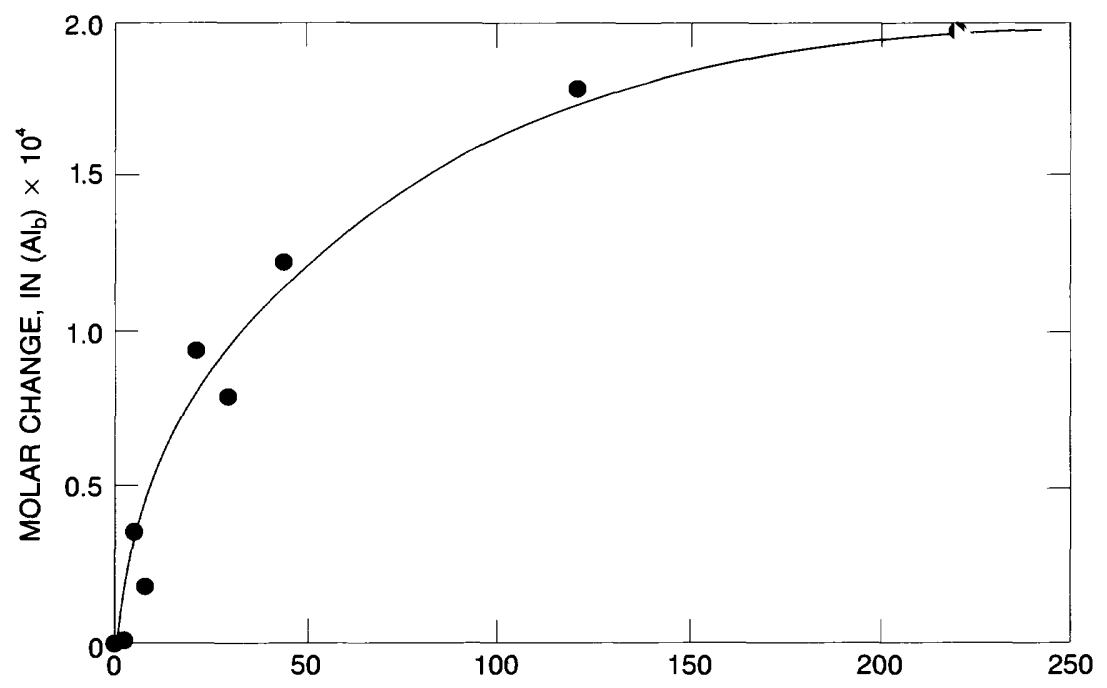

SURFACE AREA, IN METRES SQUARED PER LITRE

FigURE 9.--Initial loss of $\mathrm{Al}_{\mathrm{b}}$ due to apparent short-term adsorption from solutions $\mathrm{D}$ as a function of effective area of surface added.

Cation exchange reactions are generally rapid and can ke treated quantitatively by equilibrium chemical models. A completely quantitative treatment, however, becomes difficult in a system containing several different cationic species. In the absence of aluminum, the cation exchange positions would be occupied by sodium and hydrogen ions in the proportion dictated by $\mathrm{pH}$ and the specific behavior of the exchange sites. In a sonse, the PZC (point of zero charge) measurement characterizes the behavior of a surface toward $\mathrm{H}^{+}$; surfaces with high PZC, such as bentonite and volcanic ash, should retain $\mathrm{H}^{+}$more strongly in the neutral or mildly acid $\mathrm{pH}$ range than would be expected for the kaolinite surface which has a much lower PZC.

The CEC values for the aging-study solutions range from $0.211 \times 10^{-4}$ to $7.38 \times 10^{-4}$ equivalents per litre. The CEC is considered negligible for the solutions to which no solid was added. By comparing CEC values with the concentrations of aluminum available in the test solutions, some indication of the potential importance of ion exchange processes can be gained. In the solutions containing 0.50 $\mathrm{g}$ of volcanic ash, the CEC is sufficient to take up almo t all the aluminum as $\mathrm{Al}^{+3}$, and substantial fractions of total aluminum could be adsorbed in several other solutions. Also, the capacity for $\mathrm{H}^{+}$ adsorption is significant.

\section{SHORT-TERM ADSORPTION EXPERIMENTS}

To study more closely the rate and magnitude of the apparent initial 


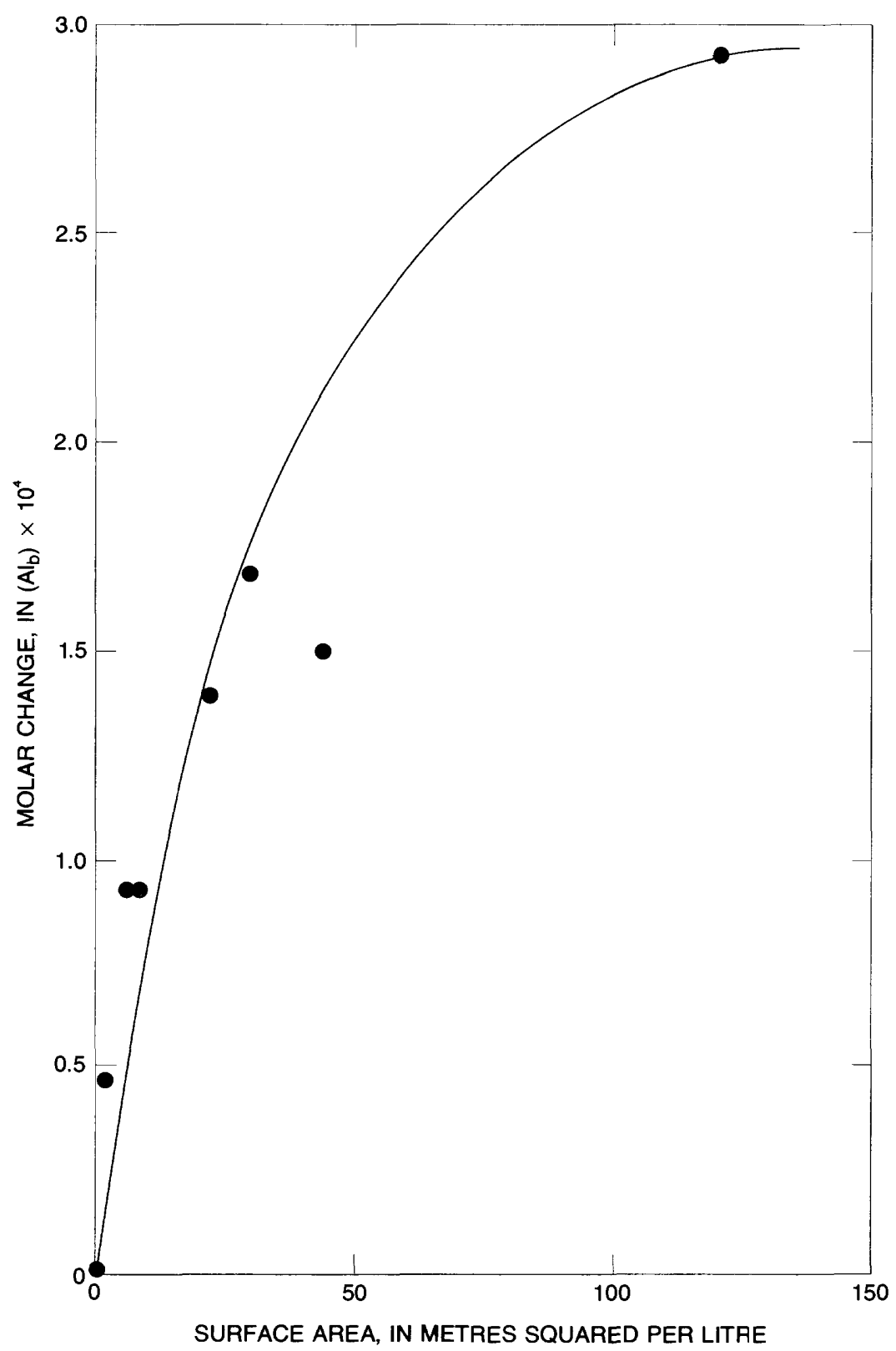

FigURE 10.- Initial loss of $\mathrm{Al}_{\mathrm{b}}$ due to apparent short-term adsorption from solutions $\mathrm{F}$ as a function of effective area of surface added.

adsorption of $\mathrm{Al}_{\mathrm{a}}$ and $\mathrm{Al}_{\mathrm{b}}, 3,000 \mathrm{ml}$ of solution $\mathrm{DS}\left(r_{n}=1.40\right)$ were made and divided into $12250-\mathrm{ml}$ aliquots. A solution having this low an $r_{n}$ 


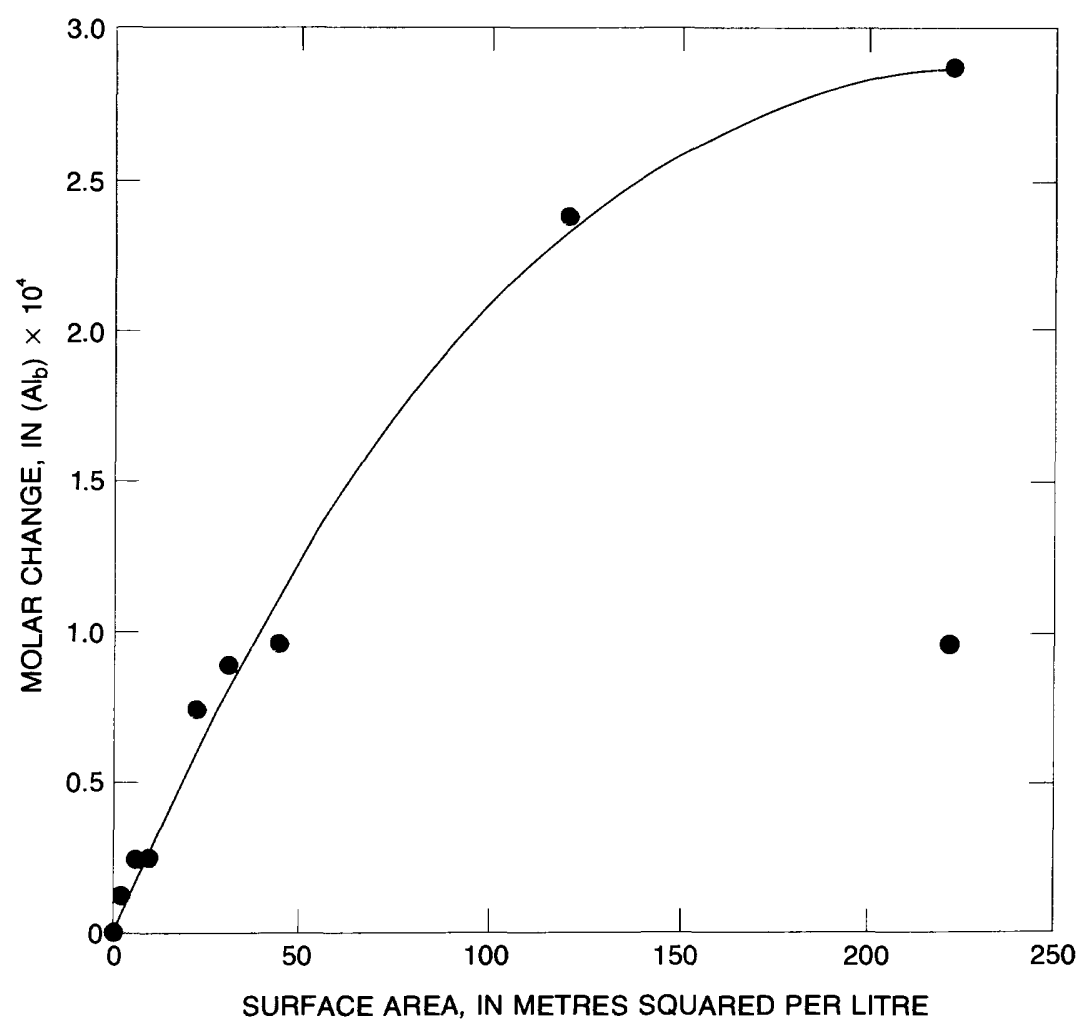

FIGURE 11.-Initial loss of $\mathrm{Al}_{\mathrm{b}}$ due to apparent short-term adsorption from solutions $\mathrm{G}$ as a function of effective area of surface added.

value was used here because the rate of $\mathrm{Al}_{\mathrm{b}}$ polymerization is slow under these conditions. Therefore, any sudden disappearar re of $\mathrm{Al}_{\mathbf{b}}$ would most likely be attributable to adsorption by the surface. The amounts of solids added were $0.010,0.030,0.10$, and 0.20 gram Wyoming bentonite, $0.020,0.050,0.10$, and $0.50 \mathrm{~g}$ volcanic ash, and 0.10 , 0.50 , and $2.0 \mathrm{~g}$ kaolinite. One solution was left untreated to act as a blank. These 12 solutions were then placed on a wrist-action shaker and shaken vigorously for 48 hours. Aliquots were withdrawn and analyzed for $\mathrm{Al}_{\mathrm{a}}$ and $\mathrm{Al}_{\mathrm{b}}$ at $1,4,32$, and 48 hours age time and also were analyzed for total aqueous aluminum at 48 hours. Thes? aliquots were centrifuged for 5 minutes at 2,000 G's prior to analysis in order to separate the suspended clays. (Comparison of $\mathrm{Al}_{\mathrm{a}}, \mathrm{Al}_{\mathrm{b}}$, and $\mathrm{Al}_{\mathrm{c}}$ analyses for a centrifuged and a noncentrifuged aliquot of the blank showed that no important quantity of aqueous aluminum was lost by this centrifuging.) They were then analyzed for $\mathrm{Al}_{\mathrm{a}}$ and $\mathrm{Al}_{\mathrm{b}}$ using the analytical procedure previously described, and aqueous $\mathrm{Al}_{\mathrm{c}}$ ras calculated by difference from the total aqueous aluminum determined after 


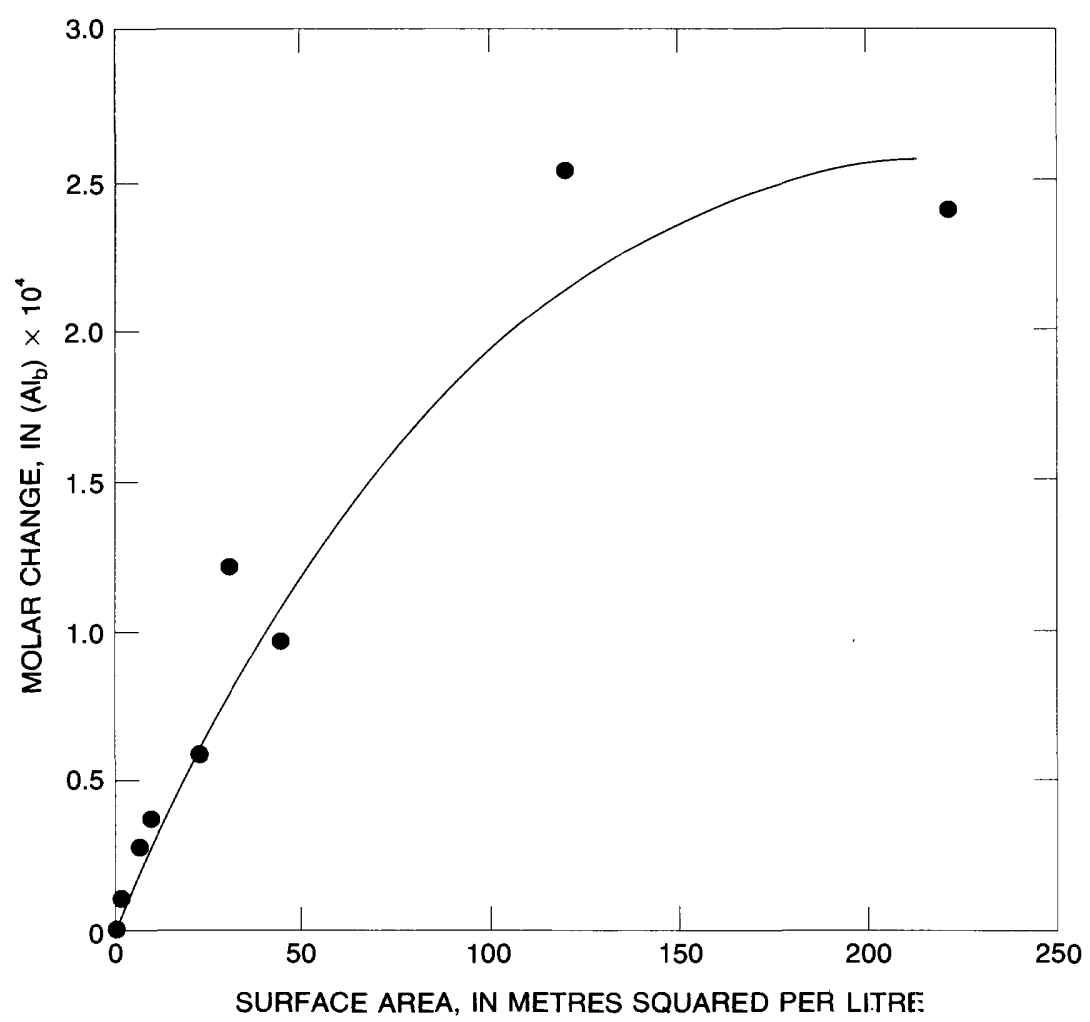

Figure 12.-Initial loss of $\mathrm{Al}_{\mathrm{b}}$ due to apparent short-term adsorption from solutions $\mathrm{H}$ as a function of effective area of surface added.

48 hours. Also at 48 hours silica content and $\mathrm{pH}$ were measured in order to determine the extent of dissolution of solids and possible interference at that time and also to be able to determine the distribution of the four $\mathrm{Al}_{\mathrm{a}}$ monomeric species. The $\mathrm{pH}$ values ranged from 4.32 for DS $2.0 \mathrm{~K}$ to 4.67 for DS $0.2 \mathrm{WB}$. The blank solution had a $\mathrm{pH}$ of 4.45. Silica concentrations at 48 hours were low, the highest being $0.18 \mathrm{mg} / 1 \mathrm{SiO}_{2}$ for DS $2.0 \mathrm{~K}$.

From these data it was concluded the dissolution of the added minerals was negligible and aluminosilicate reactions did not ineed to be considered. For each solution the amounts of $\mathrm{Al}_{\mathrm{a}}, \mathrm{Al}_{\mathrm{b}}$, and $\mathrm{Al}_{\mathrm{c}}$ adsorbed were calculated by subtracting their concentrations from those for the blank (DS NAS), defined to have zero surface area and no aluminum loss by adsorption. Although we know that loss of $\mathrm{Al}_{\mathrm{b}}$ within a 48-hour span is unlikely to be caused by increased polymerization of $\mathrm{Al}_{\mathrm{b}}$ to form more $\mathrm{Al}_{\mathrm{c}}$ as $\mathrm{Al}_{\mathrm{c}}$ is adsorbed, we may not be able to assume the same for $\mathrm{Al}_{\mathrm{a}}$ with respect to $\mathrm{Al}_{\mathrm{b}}$. Since wo have not thoroughly examined the kinetics of formation of $\mathrm{Al}_{\mathrm{b}}$ from $\mathrm{Al}_{\mathrm{a}}$, we 
have no direct experimental evidence to show whether loss of $\mathrm{Al}_{\mathrm{a}}$ is due to adsorption or to polymerization to form more $\mathrm{Al}_{\mathrm{b}}$ to compensate for the $\mathrm{Al}_{\mathrm{b}}$ adsorbed. If the latter were the case, actual adsorption of $\mathrm{Al}_{\mathrm{a}}$ would have been somewhat less and $\mathrm{Al}_{\mathrm{b}}$ adsorption somewhat more than expressed here. The methods used here to calculate adsorbed $\mathrm{Al}_{\mathrm{a}}$ and $\mathrm{Al}_{\mathrm{b}}$ may be slightly suspect in that they are based on an adsorption assumption where there is no such interaction betwoen $\mathrm{Al}_{\mathrm{a}}$ and $\mathrm{Al}_{\mathrm{b}}$ species. The experimental and calculated aluminum values are given in tables 7 and 8 .

\section{ADSORPTION OF $\mathrm{Al}_{2}$}

$\mathrm{Al}_{a}$, unlike species $\mathrm{Al}_{\mathrm{b}}$ and $\mathrm{Al}_{c}$, can be completely defined in terms of species concentrations and effective ionic charge if the $\mathrm{pH}$ is known. The concentrations of $\mathrm{Al}\left(\mathrm{H}_{2} \mathrm{O}\right)_{6}{ }^{+3}, \mathrm{AlOH}\left(\mathrm{H}_{2} \mathrm{O}\right)_{5}{ }^{+2}$, and $\mathrm{Al}(\mathrm{OH})_{2}\left(\mathrm{H}_{2} \mathrm{O}\right)_{4}{ }^{+}$are calculated from $\mathrm{Al}_{\mathrm{a}}$ concentration and $\mathrm{pH}$ using the equilibrium relationships and activity coefficients quoted earlier in this paper; $\mathrm{Al}(\mathrm{OH})_{4}\left(\mathrm{H}_{2} \mathrm{O}\right)_{2}{ }^{-}$is negligible below $\mathrm{pH}$ 5.0. The calculated values are given in table 9 . At 48 hours the $\mathrm{pH}$ values obsorved in the solutions ranged from 4.35 to 4.67 , and most of the $\mathrm{Al}_{\mathrm{a}}$ in all the solutions was in the form $\mathrm{Al}\left(\mathrm{H}_{2} \mathrm{O}\right)_{6}{ }^{+3}$ at this low $\mathrm{pH}$.

From the data on $\mathrm{Al}_{\mathrm{a}}$ and $\mathrm{pH}$ at 48 hours, it is possible to ga in some further insight into the nature of the process by which this form of aluminum is taken up by the mineral surfaces. In a general way it is obvious that solutions with the larger amounts of mineral surface area show the larger losses of $\mathrm{Al}_{\mathrm{a}}$. The relationship between surface area and loss of $\mathrm{Al}_{\mathrm{a}}$ is rather well defined, as shown by the plots in figure 13, in which the points lie close to a straight line at 48 hours and have almost the same relationship at 1 hour.

The ion exchange sorption mechanism can be explored by a mass law equilibrium model. For the systems to be examined, the cationic species present are $\mathrm{Na}^{+}, \mathrm{H}^{+}$, and the three $\mathrm{Al}_{\mathrm{a}}$ monomers, and as noted, the trivalent aluminum ion is predominant. At the $\mathrm{pH}$ range of these solutions, the sodium activity is not sufficiently great to retain a significant amount of sodium in exchange sites on the solid surfaces. Consequently, the system can be considered as involving $\mathrm{H}^{+}$and $\mathrm{Al}^{+3}$ in solution and adsorbed on the surfaces. Whether the $\mathrm{Al}^{+3}$ ion itself is reversibly adsorbed at exchange sites or the reaction is more complex, involving the conversion of $\mathrm{Al}^{+3}$ to hydroxide complexes, can also be studied, but without directly measuring all the species.

The surfaces in some of the solutions have a CEC sufficient to adsorb much more $\mathrm{H}^{+}$than the active free concentration represented by the $\mathrm{pH}$, and as noted earlier, the $\mathrm{pH}$ in some of the solutions probably shows this influence. The kaolinite surfaces may have carried enough sorbed $\mathrm{H}^{+}$when they were introduced to explain the lower $\mathrm{pH}$ 's ob- 
TABLE 7.-Adsorption experiment data

A. Molar $\mathrm{Al}_{a}$ concentration $\times 10^{4}$

\begin{tabular}{|c|c|c|c|c|}
\hline Solution & 1 hour & 4 hours & 32 hours & 48 hours \\
\hline$\overline{\text { DS NAS }}$ & 2.14 & 2.22 & 2.11 & 2.08 \\
\hline - & 2.08 & 2.18 & 2.00 & 2.06 \\
\hline DS $0.03 W B$ & 1.77 & 2.07 & 1.96 & 1.74 \\
\hline 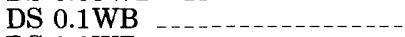 & 1.34 & 1.30 & 1.28 & 1.30 \\
\hline - & .39 & .26 & .31 & .45 \\
\hline DS $0.02 \mathrm{VA}$ & 2.06 & 2.16 & 2.03 & 2.06 \\
\hline DS $0.05 \mathrm{VA}$ & 2.01 & 2.15 & 1.97 & 2.05 \\
\hline DS $0.1 \mathrm{VA}$ & 1.83 & 1.85 & 1.87 & 1.91 \\
\hline DS $0.5 \mathrm{VA}$ & 1.02 & 1.10 & 1.12 & 1.12 \\
\hline DS $0.1 \mathrm{~K}$ & 1.95 & 2.11 & 1.97 & 1.96 \\
\hline DS $0.5 \mathrm{~K}$ & 1.91 & 2.02 & 1.91 & 1.92 \\
\hline $\mathrm{DS} 2.0 \mathrm{~K}$ & 1.70 & 1.81 & 1.86 & 1.86 \\
\hline
\end{tabular}

B. Molar $\mathrm{Al}_{b}$ concentration $\times 10^{4}$

\begin{tabular}{|c|c|c|c|c|}
\hline Solution & 1 hour & 4 hours & 32 hours & 48 hours \\
\hline DS NAS & 1.87 & 1.84 & 1.90 & 1.94 \\
\hline - & 1.51 & 1.45 & 1.54 & 1.54 \\
\hline 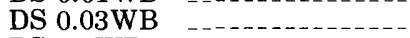 & .96 & .69 & .75 & .70 \\
\hline DS $0.1 \mathrm{WB}$ & .11 & .14 & .17 & .14 \\
\hline DS $0.2 \mathrm{WB}$ & .06 & .03 & .02 & .01 \\
\hline DS $0.02 \mathrm{VA}_{\ldots}$ & 1.63 & 1.61 & 1.65 & 1.63 \\
\hline DS $0.05 V A_{\ldots}$ & 1.53 & 1.38 & 1.38 & 1.38 \\
\hline DS $0.1 \mathrm{VA}$ & 1.22 & .99 & .98 & .92 \\
\hline DS $0.5 \mathrm{VA}$ & .18 & .15 & .13 & .13 \\
\hline DS $0.1 \mathrm{~K}$ & 1.79 & 1.60 & 1.77 & 1.77 \\
\hline $\mathrm{DS} 0.5 \mathrm{~K}$ & 1.51 & 1.40 & 1.42 & 1.27 \\
\hline $\mathrm{DS} 2.0 \mathrm{~K}$ & .42 & .34 & .36 & .29 \\
\hline
\end{tabular}

C. Other analyses at 48 hours

\begin{tabular}{|c|c|c|c|}
\hline Solution & $\begin{array}{c}\text { Total aqueous } \mathrm{Al} \text {, } \\
\text { molar } \times 10^{4}\end{array}$ & $\mathrm{pH}$ & $\begin{array}{c}\mathrm{SiO}_{2} \\
(\mathrm{mg} / \mathrm{l}) \\
\end{array}$ \\
\hline DS NAS & 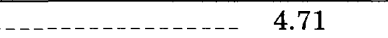 & 4.46 & 0.00 \\
\hline $\mathrm{DS} 0.01 \mathrm{WB}$ & - & 4.45 & .03 \\
\hline DS $0.03 \mathrm{WB}$ & - & 4.47 & .01 \\
\hline DS $0.1 \mathrm{WB}$ & 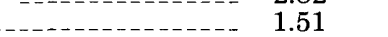 & 4.46 & .05 \\
\hline $\mathrm{DS} 0.2 \mathrm{WB}$ & - n & 4.67 & .03 \\
\hline $\mathrm{DS} 0.02 \mathrm{VA}$ & - & 4.48 & .02 \\
\hline DS $0.05 \mathrm{VA}$ & - & 4.45 & .02 \\
\hline DS $0.1 \mathrm{VA}$ & (n) & 4.44 & .04 \\
\hline DS $0.5 \mathrm{VA}$ & - & 4.41 & .07 \\
\hline DS $0.1 \mathrm{~K}$ & - & 4.48 & .02 \\
\hline $\mathrm{DS} 0.5 \mathrm{~K}$ & 3.80 & 4.40 & .06 \\
\hline DS $2.0 \mathrm{~K}$ & 2.25 & 4.35 & .18 \\
\hline
\end{tabular}

TABLE 8.-Calculated sorbed species and aqueous $\mathrm{Al}_{c}$ concentrations

[Concentrations expressed in moles/litre $\times 10^{4}$; sorbed species are expressed in terms of the concentration change effected by the addition of the surface. For solution DS NAS, sorbed concentration is defined as zero for all species]

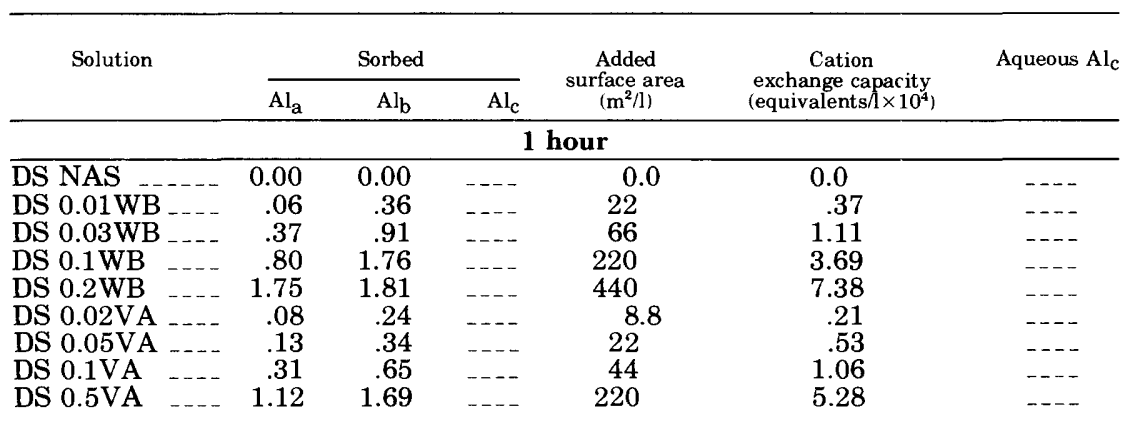




\begin{tabular}{|c|c|c|c|c|c|c|}
\hline \multirow[t]{2}{*}{ Solution } & \multicolumn{3}{|c|}{ Sorbed } & \multirow{2}{*}{$\begin{array}{c}\text { Added } \\
\text { surface area } \\
\left(\mathrm{m}^{2} / 1\right) \\
\end{array}$} & \multirow{2}{*}{$\begin{array}{c}\text { Cation } \\
\text { exchange capacity } \\
\text { (equivalents } \Lambda \times 10^{4} \text { ) }\end{array}$} & \multirow[t]{2}{*}{ Aqueous $\mathrm{Al}_{\mathrm{C}}$} \\
\hline & $\mathrm{Al}_{\mathrm{a}}$ & $\mathrm{Al}_{\mathbf{b}}$ & $\mathrm{Al}_{\mathrm{C}}$ & & & \\
\hline \multicolumn{7}{|c|}{1 hour-Continued } \\
\hline DS $0.1 \mathrm{~K}$ & .19 & .08 & $\ldots$ & 6.0 & 0.28 & $-\ldots$ \\
\hline DS $0.5 \mathrm{~K}$ & .23 & .36 & $---\ldots$ & 30 & 1.38 & --- \\
\hline $\mathrm{DS} 2.0 \mathrm{~K}$ & .44 & 1.45 & --- & 120 & 5.52 & ---- \\
\hline \multicolumn{7}{|c|}{4 hours } \\
\hline DS NAS & 0.00 & 0.00 & $-\ldots$ & --- & $\ldots$ & $\ldots$ \\
\hline DS 0.01WB & .04 & .39 & --- & --- & ---- & --- \\
\hline DS $0.03 W^{-}$ & .15 & 1.15 & --- & --- & $\ldots-$ & --- \\
\hline DS 0.1WB & .92 & 1.70 & --- & --- & $-\ldots$ & ---- \\
\hline DS $0.2 \mathrm{WB}$ & 1.96 & 1.81 & $\ldots$ & $-\ldots$ & $-\cdots$ & --- \\
\hline DS $0.02 \mathrm{VA}$ & .06 & .23 & --- & $\ldots--$ & $--\infty$ & --- \\
\hline DS $0.05 \mathrm{VA}$ & .07 & .46 & --- & --- & $-\ldots$ & $-\cdots$ \\
\hline DS 0.1VA & .37 & .85 & --- & --- & $\ldots$ & $-\ldots$ \\
\hline DS $0.5 \mathrm{VA}$ & 1.12 & 1.69 & --- & --- & $\ldots$ & --- \\
\hline DS $0.1 \mathrm{~K}$ & .11 & .24 & --- & ---- & $-\ldots$ & --- \\
\hline DS $0.5 \mathrm{~K}$ & .20 & .44 & ---- & ---- & $\ldots-$ & ---- \\
\hline DS 2.0K & .41 & 1.50 & $\ldots$ & $\ldots$ & $\ldots$ & --- \\
\hline \multicolumn{7}{|c|}{32 hours } \\
\hline DS NAS & 0.00 & 0.00 & ---- & $\ldots$ & --- & ---- \\
\hline DS $0.01 \mathrm{WB}$ & .11 & .36 & --- & --- & --- & --- \\
\hline DS 0.03WB & .15 & 1.15 & $-\ldots$ & ---- & ---- & --- \\
\hline DS $0.1 \mathrm{WB}$ & .83 & 1.73 & --- & --- & --- & --- \\
\hline DS 0.2WB & 1.80 & 1.88 & --- & --- & --- & ---- \\
\hline DS $0.02 \mathrm{VA}$ & .08 & .25 & --- & $-\ldots$ & --- & --- \\
\hline DS $0.05 \mathrm{VA}$ & .14 & .52 & --- & --- & --- & --- \\
\hline DS 0.1VA & .24 & .92 & --- & --- & --- & $-\infty-$ \\
\hline DS $0.5 \mathrm{VA}$ & .99 & .77 & $-\cdots$ & --- & --- & $-\cdots$ \\
\hline DS $0.1 \mathrm{~K}$ & .14 & .13 & $--\cdots$ & --- & --- & --- \\
\hline DS $0.5 \mathrm{~K}$ & .20 & .48 & $\ldots-$ & --- & $\ldots-$ & $\ldots-$ \\
\hline DS 2.0K & .25 & 1.54 & --- & ---- & ---- & ---- \\
\hline \multicolumn{7}{|c|}{48 hours } \\
\hline DS NAS & 0.00 & 0.00 & 0.00 & --- & $\ldots-$ & 0.69 \\
\hline DS $0.01 \mathrm{WB}$ & .02 & .40 & .09 & --- & $-\ldots$ & .60 \\
\hline DS $0.03 \mathrm{WB} \ldots$ & .34 & 1.24 & .31 & $-\ldots$ & ---- & .38 \\
\hline DS $0.1 \mathrm{WB}$ & .78 & 1.80 & .62 & $-\ldots$ & --- & .07 \\
\hline DS $0.2 \mathrm{WB}$ & 1.63 & 1.93 & .69 & $-\ldots$ & ---- & .00 \\
\hline DS $0.02 \mathrm{VA}$ & .02 & .30 & .07 & $-\ldots$ & $\ldots--$ & .61 \\
\hline DS $0.05 \mathrm{VA}$ & .03 & .56 & .34 & --- & ---- & .35 \\
\hline DS 0.1VA & .17 & 1.02 & .35 & $\ldots-$ & --- & .34 \\
\hline DS $0.5 \mathrm{VA}$ & .96 & 1.81 & .57 & $-\ldots$ & $\ldots$ & .12 \\
\hline DS $0.1 \mathrm{~K}$ & .12 & .17 & .08 & $\ldots$ & $-\ldots$ & .61 \\
\hline DS $0.5 \mathrm{~K}$ & .16 & .67 & .08 & --- & ---- & .61 \\
\hline DS 2.0K & .22 & 1.65 & .59 & & $\ldots$ & .10 \\
\hline
\end{tabular}

TABLE 9.-Calculated aqueous $\mathrm{Al}_{a}$ species distribution at 48 hours

Molar concentration $\times 10^{4}$

\begin{tabular}{|c|c|c|c|c|c|}
\hline Solution & $\mathrm{pH}$ & $\left(\mathrm{Al}_{\mathrm{a}}\right)$ & $(\mathrm{Al}+3)$ & $(\mathrm{AlOH}+2)$ & $\left(\mathrm{Al}(\mathrm{OH})_{2}+\right)$ \\
\hline DS NAS & 4.46 & 2.08 & 1.67 & 0.30 & 0.11 \\
\hline DS $0.01 \mathrm{WB}$ & 4.45 & 2.06 & 1.65 & .30 & .11 \\
\hline DS 0.03WB & 4.47 & 1.74 & 1.38 & .26 & .10 \\
\hline DS $0.1 \mathrm{WB}$ & 4.46 & 1.30 & 1.04 & .19 & .07 \\
\hline DS $0.2 \mathrm{WB}$ & 4.67 & .45 & .31 & .09 & .05 \\
\hline $\mathrm{DS} 0.02 \mathrm{VA}$ & 4.48 & 2.06 & 1.65 & .30 & .11 \\
\hline DS $0.05 \mathrm{VA}$ & 4.45 & 2.05 & 1.64 & .30 & .11 \\
\hline $\mathrm{DS} 0.1 \mathrm{VA}$ & 4.44 & 1.91 & 1.55 & .27 & .09 \\
\hline $\mathrm{DS} 0.5 \mathrm{VA}$ & 4.41 & 1.12 & .92 & .15 & .05 \\
\hline $\mathrm{DS} 0.1 \mathrm{~K}$ & 4.48 & 1.96 & $1.5 \overline{5}$ & .30 & .11 \\
\hline $\mathrm{DS} 0.5 \mathrm{~K}$ & 4.40 & 1.92 & 1.59 & .25 & .08 \\
\hline $\mathrm{DS} 2.0 \mathrm{~K}$ & 4.35 & 1.86 & 1.58 & .22 & .06 \\
\hline
\end{tabular}




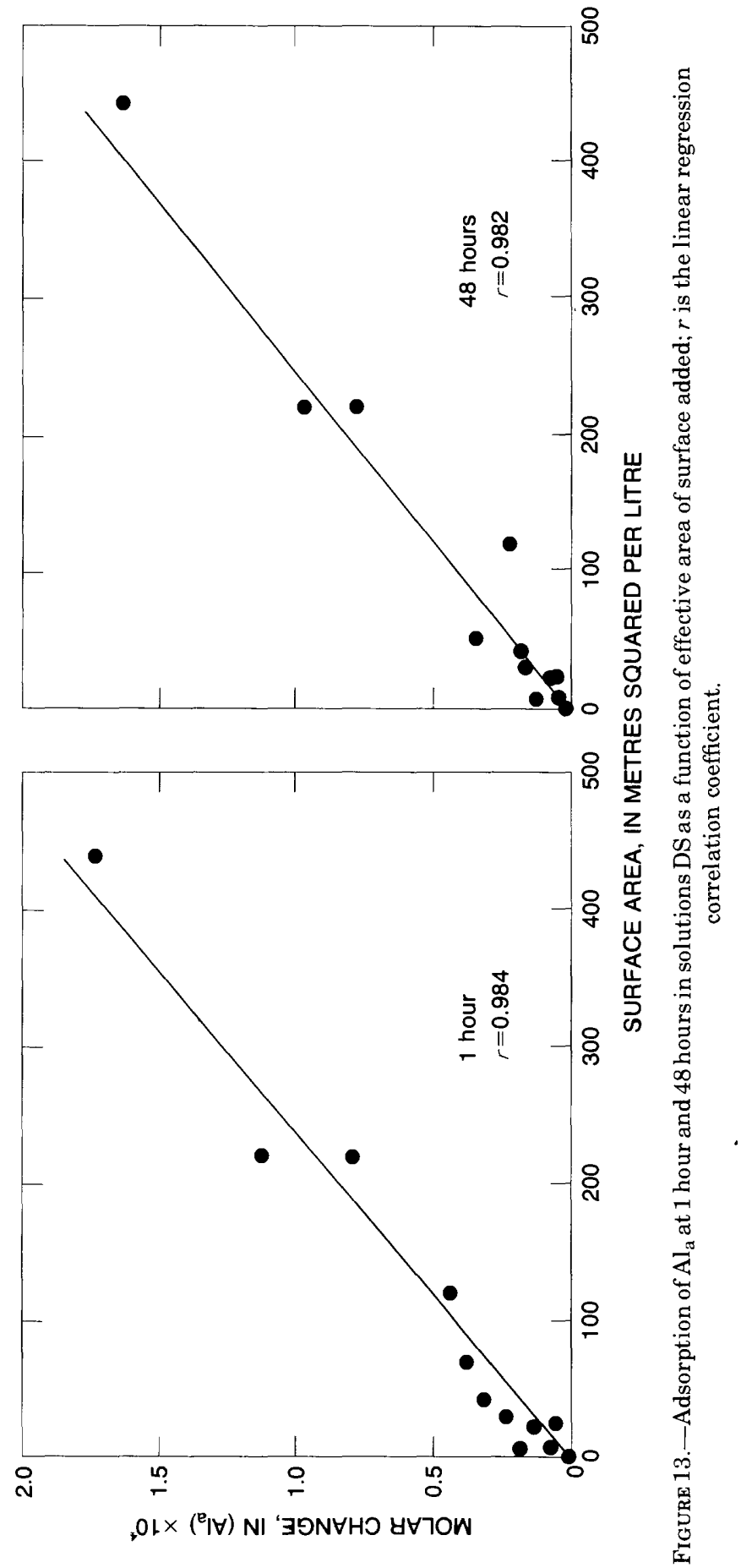


served in solutions DS $2.0 \mathrm{~K}$ and DS $0.5 \mathrm{~K}$, and some of the other surfaces may have had other ions in exchange sites that were renlaced by $\mathrm{H}^{+}$when the solutions were prepared. The higher $\mathrm{pH}$ of solution DS $0.2 \mathrm{WB}$ might, for example, have been related to exchange of adsorbed $\mathrm{Na}^{+}$for $\mathrm{H}^{+}$. In any event, the equilibrium model, if valid, should be capable of treating $\mathrm{H}^{+}-\mathrm{Al}^{+3}$ relationships regardless of the cutside influences on $\mathrm{pH}$.

In its simplest form the cation exchange equilibrium might be written

$$
\mathrm{Al}^{+3}+3 \mathrm{H}_{x \curvearrowleft} \rightleftharpoons 3 \mathrm{H}^{+}+\mathrm{Al}_{x 3}
$$

and

$$
\frac{\left[\mathrm{H}^{+}\right]^{3}\left(\mathrm{Al}_{x 3}\right)}{\left[\mathrm{Al}^{+3}\right]\left(\mathrm{H}_{x}\right)^{3}}=K_{D 3} .
$$

The $x$ terms represent adsorbed material, expressed as concentrations in moles per litre. For solutions containing the amounts of the solids shown, the CEC in equivalents per litre is known and can be expressed as

$$
\mathrm{CEC}=3\left(\mathrm{Al}_{x 3}\right)+\left(\mathrm{H}_{x}\right) .
$$

The amounts of adsorbed $\mathrm{Al}_{\mathrm{a}}$ shown in the 11 solutions in table 9 were considered to represent $\mathrm{Al}_{x}$; therefore, the amount of $\mathrm{H}_{x}$ can be calculated for each solution. The value of $\left[\mathrm{Al}^{+3}\right]$ was computed by multiplying the determined concentration of $\mathrm{Al}^{+}+3$ by the appropriate $\varepsilon$ ctivity coefficient for these solutions. If ionic strength is near 0.01, $\gamma_{\mathrm{Al}^{+3}}=0.45$. Results of the calculation for $K_{D 3}$ (table 10) show a wide range $\left(10^{-3.62}\right.$ to $\left.10^{+1.45}\right)$ for what is supposed to be a constant. It may be concluded, therefore, that the form of the exchange equilibrium used was not applicable.

The alteration of $\mathrm{Al}^{+3}$ to a hydroxide complex at the surface at the time of adsorption could be represented by the equation

$$
\mathrm{Al}^{+3}+\mathrm{H}_{2} \mathrm{O}+2 \mathrm{H}_{x} \rightleftharpoons \mathrm{AlOH}_{x 2}+3 \mathrm{H}^{+}
$$

or

where

$$
\frac{\left[\mathrm{H}^{+}\right]^{3}\left(\mathrm{AlOH}_{x 2}\right)}{\left[\mathrm{Al}^{+3}\right]\left(\mathrm{H}_{x}\right)^{2}}=K_{D 2} \text { on this system, }
$$

$$
\mathrm{CEC}=2\left(\mathrm{AlOH}_{x 2}\right)+\left(\mathrm{H}_{x}\right)
$$

and a recalculation of $\left(\mathrm{H}_{x}\right)$ is needed. Using this new value for $\left(\mathrm{H}_{x}\right)$ and the mass law equation for $K_{D 2}$, a set of $K$ values is obtained with a 
TABLE 10.-Dissolved and sorbed species and selectivity constants for three ion exchange mechanisms (solutions aged 48 hours)

\begin{tabular}{|c|c|c|c|c|c|}
\hline Solution & {$\left[\mathrm{H}^{+}\right]$} & {$[\mathrm{Al}+3]$} & $\mathrm{Al}_{\alpha x}$ & $\mathrm{H}_{x}$ & $K_{D}$ \\
\hline DS NAS & $10^{-4.46}$ & $10^{-4.13}$ & & & \\
\hline DS $0.01 \mathrm{WB}$ & 3 & $10^{-4.13}$ & $10^{-5.70}$ & $\begin{array}{l}10^{-4.51} \\
10^{-4.48}\end{array}$ & $\begin{array}{l}10^{-1.39} \\
10^{-5.96}\end{array}$ \\
\hline DS $0.03 \mathrm{WB}$ & $3 \ldots 10^{-4.47}$ & $10^{-4.21}$ & $10^{-4.47}$ & $\begin{array}{l}10^{-4.46} \\
10^{-5.04} \\
10^{-4.37} \\
10^{-4.11}\end{array}$ & $\begin{array}{l}10^{-10.46} \\
10^{+1.45} \\
10^{-4.93} \\
10^{-9.64}\end{array}$ \\
\hline DS $0.1 \mathrm{WB}$ & $10^{-4.46}$ & $10^{-4.33}$ & $10^{-4.11}$ & $\begin{array}{l}10^{-3.87} \\
10^{-3.67} \\
10^{-3.54}\end{array}$ & $\begin{array}{l}10^{-1.55} \\
10^{-5.82} \\
10^{-9.62}\end{array}$ \\
\hline $\mathrm{DS} 0.2 \mathrm{WB}$ & $10^{-4.67}$ & $10^{-4.86}$ & $10^{-3.79}$ & $\begin{array}{l}10^{-3.60} \\
10^{-3.38} \\
10^{-3.24}\end{array}$ & $\begin{array}{l}10^{-2.14} \\
10^{-6.18} \\
10^{-9.70}\end{array}$ \\
\hline $\mathrm{DS} 0.02 \mathrm{VA}$ & $10^{-4.48}$ & $10^{-4.13}$ & $10^{-5.70}$ & $\begin{array}{l}10^{-4.83} \\
10^{-4.77} \\
10^{-4.72}\end{array}$ & $\begin{array}{l}10^{-} .52 \\
10^{-5.47} \\
10^{-10.29}\end{array}$ \\
\hline DS $0.05 \mathrm{VA}$ & $10^{-4.45}$ & $10^{-4.14}$ & $10^{-5.52}$ & $\begin{array}{l}10^{-4.36} \\
10^{-4.33} \\
10^{-4.30}\end{array}$ & $\begin{array}{l}10^{-1.65} \\
10^{-6.07} \\
10^{-10.43}\end{array}$ \\
\hline DS $0.1 \mathrm{VA}_{\ldots}$ & $10^{-4.44}$ & $10^{-4.16}$ & $10^{-4.77}$ & $\begin{array}{l}10^{-4.26} \\
10^{-4.14} \\
10^{-4.05}\end{array}$ & $\begin{array}{l}10^{-1.15} \\
10^{-5.65} \\
10^{-9.88}\end{array}$ \\
\hline $\mathrm{DS} 0.5 \mathrm{VA}_{-}$ & $10^{-4.41}$ & $10^{-4.39}$ & $10^{-4.02}$ & $\begin{array}{l}10^{-3.62} \\
10^{-3.47} \\
10^{-3.36}\end{array}$ & $\begin{array}{l}10^{-2.00} \\
10^{-5.95} \\
10^{-9.50}\end{array}$ \\
\hline $\mathrm{DS} 0.1 \mathrm{~K}$ & $10^{-4.48}$ & $10^{-4.16}$ & $10^{-4.92}$ & $\begin{array}{l}<0 \\
10^{-5.44} \\
10^{-4.85}\end{array}$ & $10^{-3.32}$ \\
\hline $\mathrm{DS} 0.5 \mathrm{~K}$ & $10^{-4.40}$ & $10^{-4.15}$ & $10^{-4.80}$ & $\begin{array}{l}10^{-4.05} \\
10^{-3.97} \\
10^{-3.91}\end{array}$ & $\begin{array}{l}10^{-1.70} \\
10^{-5.91} \\
10^{-9.94}\end{array}$ \\
\hline $\mathrm{DS} 2.0 \mathrm{~K}$ & $10^{-4.35}$ & $10^{-4.16}$ & $10^{-4.66}$ & $\begin{array}{l}10^{-3.31} \\
10^{-3.29} \\
10^{-3.27} \\
\end{array}$ & $\begin{array}{l}10^{-3.62} \\
10^{-6.17} \\
10^{-10.28}\end{array}$ \\
\hline
\end{tabular}

range of $10^{-6.18}$ to $10^{-3.32}$. This spread is not greatly decreased from the one obtained for $K_{D 3}$.

A further alteration of the equilibrium equation can be made, however, which perhaps is more in accord with what might normally be expected, in that a single $\mathrm{Al}^{+3}$ ion is unlikely to neutralize two or three unit surface charges which could be spread some distance apart over the surface. Attachment of the $\mathrm{Al}^{+3}$ ion at a single charge site can be represented as

$$
\begin{gathered}
\mathrm{Al}^{+3}+2 \mathrm{H}_{2} \mathrm{O}+\mathrm{H}_{x} \rightleftharpoons \mathrm{Al}(\mathrm{OH})_{2 x}+3 \mathrm{H}^{+}, \\
\frac{\left.\left[\mathrm{H}^{+}\right]^{3} \mathrm{Al}(\mathrm{OH})_{2 x}\right)}{\mathrm{Al}+3\left(\mathrm{H}_{x}\right)}=K_{D 1},
\end{gathered}
$$

and

$$
\mathrm{CEC}=\left(\mathrm{Al}(\mathrm{OH})_{2 x}\right)+\left(\mathrm{H}_{x}\right) .
$$


Recalculation of $\left(\mathrm{H}_{x}\right)$ using this model and solving for $K_{D 1}$ gave values ranging from $10^{-10.46}$ to $10^{-9.50}$. Although the "constant" still has a fairly wide range, the improvement obtained by postulating a 1:1 exchange of $\mathrm{H}^{+}$for $\mathrm{Al}^{+3}$ is obvious and suggests that the process takes place in some such fashion. Numerous references in the literature relate the apparent preference for hydroxide complexes over uncomplexed species where polyvalent metals are being adsorbed at inorganic surfaces.

In addition to the cation adsorption process, the activities of $\mathrm{Al}^{+3}$ and $\mathrm{H}^{+}$also are influenced in these solutions by the precipitation of gibbsite. When the exchange capacity is small compared with the total aluminum present in the system, the adsorbed $\mathrm{Al}_{\mathrm{a}}$ species will be withheld at the expense of $\mathrm{Al}_{\mathrm{c}}$, the precipitated form, or $\mathrm{Al}_{\mathrm{b}}$, the metastable potentially precipitable form. Thus at equilibrium both the adsorbed and solid forms could be present. Some of the solutions in this study contained an exchange capacity a little greater than the total molar concentration of aluminum, but the amount of $\mathrm{Al}_{\mathrm{a}}$ sirbed at the $\mathrm{pH}$ of the solutions was never large enough to preclude gil bsite precipitation. The adsorption of $\mathrm{H}^{+}$by the solids tends to obscure the stoichiometry of $\mathrm{Al}^{+3}$ and $\mathrm{OH}^{-}$species involved in complexing or polymerization, and the $\mathrm{pH}$ changes in solutions with solid surfaces are not comparable with those where surfaces are absent.

Effects of mineral surfaces on $\mathrm{Al}_{\mathrm{a}}$ concentrations can be summarized as follows,

1. $\mathrm{Al}_{\mathrm{a}}$ species are adsorbed by the mineral surfaces, and the process appears to follow a cation exchange mass law equilibrium model.

2. The adsorption of $\mathrm{Al}_{\mathrm{a}}$ is essentially complete in 1 hour of reaction time.

3. Adsorbed $\mathrm{Al}_{\mathrm{a}}$ is produced within the system at the expense of precipitated forms of $\mathrm{Al}$ when the solution is initially supersaturated with respect to gibbsite. Gibbsite solubility will control $\mathrm{Al}_{\mathrm{a}}$ concentration ultimately unless the exchange capacity is large enough to remove almost all $\mathrm{Al}_{\mathrm{a}}$. Where a large excess of exchange capacity is present, the $\mathrm{Al}_{\mathrm{a}}$ concentration may be maintained at a level governed by ion exchange equilibria.

4. Adsorbed $\mathrm{Al}_{\mathrm{a}}$ evidently can be desorbed and returned to solution more readily than precipitated $\mathrm{Al}_{\mathrm{c}}$ can be redissolved or than polymerized $\mathrm{Al}_{\mathrm{b}}$ can be degraded to $\mathrm{Al}_{\mathrm{a}}$.

5 . In the process of being adsorbed, $\mathrm{Al}_{\mathrm{a}}$ species appear to bocome essentially monovalent, perhaps through formation of hydroxide complexes at one or more sites in the hydration shell of the $\mathrm{Al}^{+3}$ form. In any event, the processes of adsorption and hydrolysis or the first stages of polymerization of $\mathrm{Al}_{\mathrm{a}}$ are closely intermin gled. 


\section{ADSORPTION OF $\mathbf{A l}_{\mathrm{b}}$}

Data in table 7 show that in all the 11 solutions that contained mineral surfaces there was significantly less $\mathrm{Al}_{b}$ than in the blank. It is also evident that the loss of $\mathrm{Al}_{\mathrm{b}}$ had already mostly taken place before the first set of determinations were made 1 lour after the solutions were prepared and that the changes in aqueous $\mathrm{Al}_{\mathrm{b}}$ concentrations are greater on addition of the minerals for the solutions having the greater surface areas. These concentration changes could have resulted from adsorption of $\mathrm{Al}_{b}$ species by the surfaces, or the surfaces might have promoted or catalyzed the polymerization of $\mathrm{Al}_{\mathrm{b}}$ to form crystalline gibbsite.

If the latter hypothesis were correct, one would expect a lower $\mathrm{pH}$ and a closer approach to the equilibrium solubility of gibbsite in solutions having the larger surface areas and CEC's. In figure 14 the $\log$ of the calculated solubility product for $\mathrm{Al}(\mathrm{OH})_{3}$ for each solution is plotted against the log of the CEC in that solution. Although these points are rather scattered, they suggest a trend toward a more stable $\mathrm{Al}(\mathrm{OH})_{3}$ solid is produced by increasing the CEC. For the blank, the value for $\mathrm{K}_{s 0}$ is $10^{-32.75}$, essentially that of microcrystalline gibbsite.

Although the number of points is rather small, figure 14 does indicate that the three materials have somewhat different effects. Both the kaolinite and volcanic ash surfaces show, as CEC increases, a more

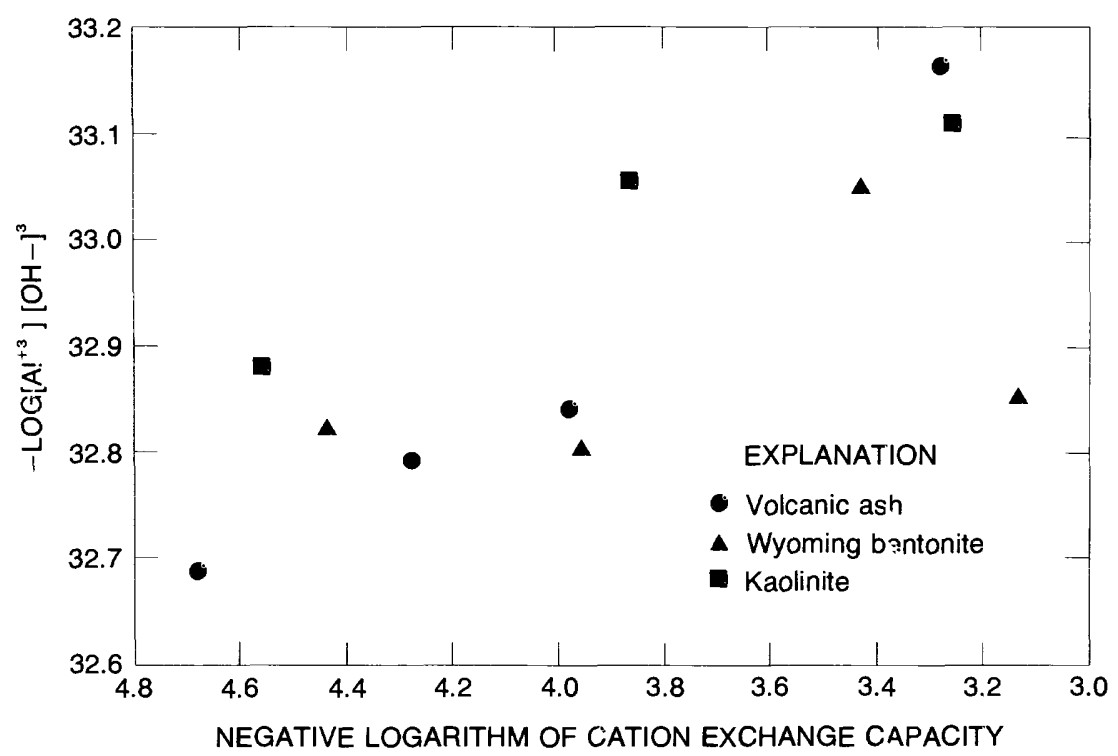

FIGURE 14.-Gibbsite ion activity product in solutions DS as a function of negative logarithm of equivalents of cation exchange capacity added per litre of solution. 
strongly decreasing trend in $\mathrm{K}_{s 0}$ than does the bentonite surface. However, the significance of the apparent correlation is limited and uncertain, because the $\mathrm{K}_{s 0}$ values for the longer term aging-study solutions do not display a discernihle pattern. It can only be concluded that the surfaces may have influenced the early stages of the reactions that precipitated $\mathrm{Al}(\mathrm{OH})_{3}$.

Plots of the changes in concentrations of $\mathrm{Al}_{\mathrm{b}}$ as functions of surface area and of CEC (figs. 15, 16) indicate that the adsorption of $\mathrm{Al}_{b}$ is somewhat more closely related to surface area than to CEC. This seems reasonable, because $\mathrm{Al}_{b}$ units are large polymers with a considerable range in net positive charge per included aluminum atom. They cannot be expected to exhibit the same behavior as $\mathrm{Al}_{\mathrm{a}}$ species wherein each individual aluminum atom is bound to a charge site. We should, therefore, not expect the adsorbed $\mathrm{Al}_{\mathrm{b}}$ to be strictly dependent on the number of surface charge sites, or CEC. The relationship between $\mathrm{Al}_{\mathrm{b}}$ adsorption and surface area is linear if $\mathrm{Al}_{b}$ remains in solution in substantial excess. Complete adsorption of $\mathrm{Al}_{\mathrm{b}}$ is approached asymptotically if an excess of surface area is present. This behavior is characteristic of adsorption equilibria.

Unlike the adsorption of $\mathrm{Al}_{\mathrm{a}}$, some of the adsorption of $\mathrm{Al}_{\mathrm{b}}$ occurs between 1 and 48 hours. The slopes of the linear portions of the adsorption-surface area plots at 1 and 48 hours are $1.3 \times 10^{-6}$ and $2.2 \times 10^{-6}$ moles $\mathrm{Al}_{\mathrm{b}} / \mathrm{m}^{2}$, respectively. For those solutions in which there is a large excess of $\mathrm{Al}_{\mathrm{b}}$, about 40 percent of the adsorption occurs between 1 and 48 hours. A possible explanation for the longer time of completion of adsorption of $\mathrm{Al}_{b}$ is that the $\mathrm{Al}_{b}$ polymers, being larger and having less charce per aluminum atom than $\mathrm{Al}_{\mathrm{a}}$, are less mobile. Figure 17, the plot of adsorption of $\mathrm{Al}_{\mathrm{b}}$ against the time of contact between the solutic ns and the surfaces, shows how quickly the adsorption does occur. Seemingly, it would completely stabilize after several days, thereby allowing us to account for $\mathrm{Al}_{\mathrm{b}}$ polymerization as the sole process causing disappearance of $\mathrm{Alb}$ after those several days. At 48 hours, the slope of $\mathrm{Al}_{\mathrm{b}}$ adsorption, $2.2 \times 10^{-6} \mathrm{moles} / \mathrm{m}^{2}$, is conciderably larger than the slope of $\mathrm{Al}_{a}$ adsorption at that time, $3.0 \times 10^{-7}$ moles $/ \mathrm{m}^{2}$. This is in general agreement with many observatic $n$ s in the literature which report strong adsorption effects for polynuclear ions; hov, ever, the polynuclear species are generally large units, each containing many metal ions. Thus, each $\mathrm{Al}_{\mathrm{b}}$ unit that is adsork od has a much larger effect on analytically determined aluminum than does the adsorption of a single aluminum monomer.

The $\mathrm{Al}_{b}$ units represent a range of compositions, with a net charge per aluminum atom that probably is commonly less than one, and so an ion exchange equilibrium model probably cannot be expected to be appropriate for this kind of material. Also, the techniques used by 


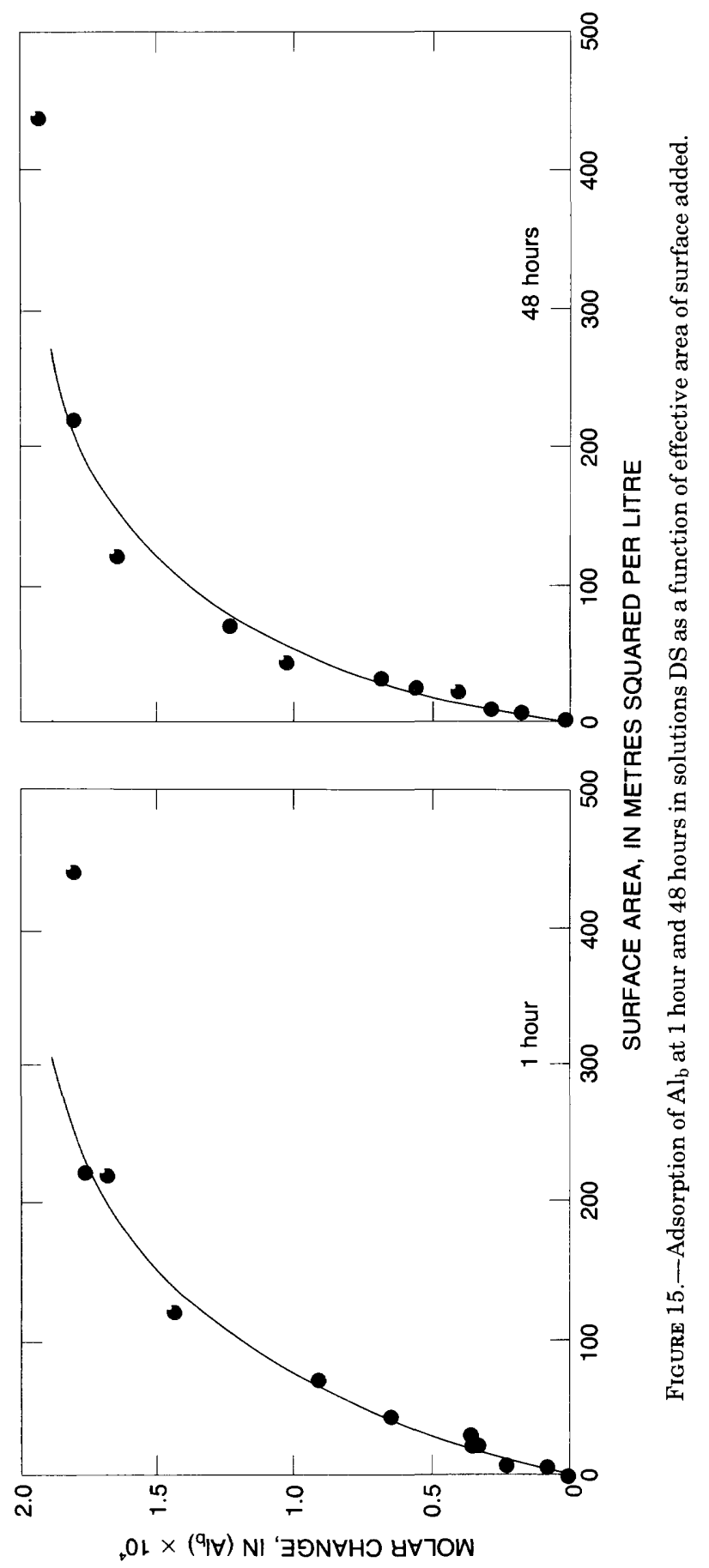




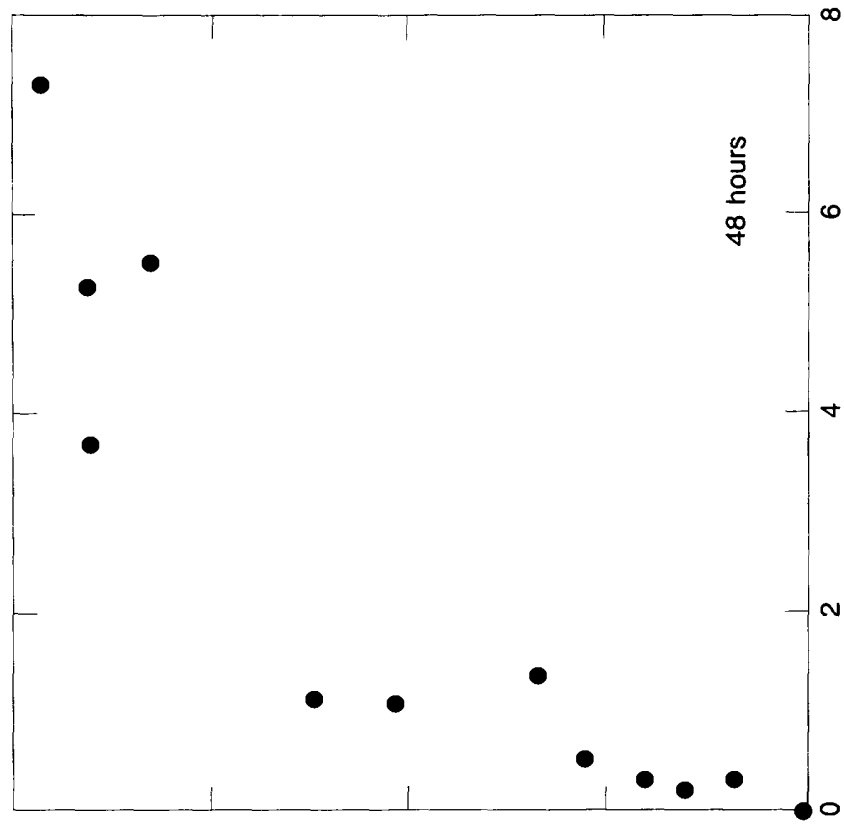

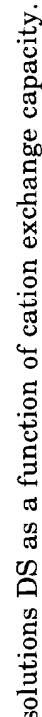

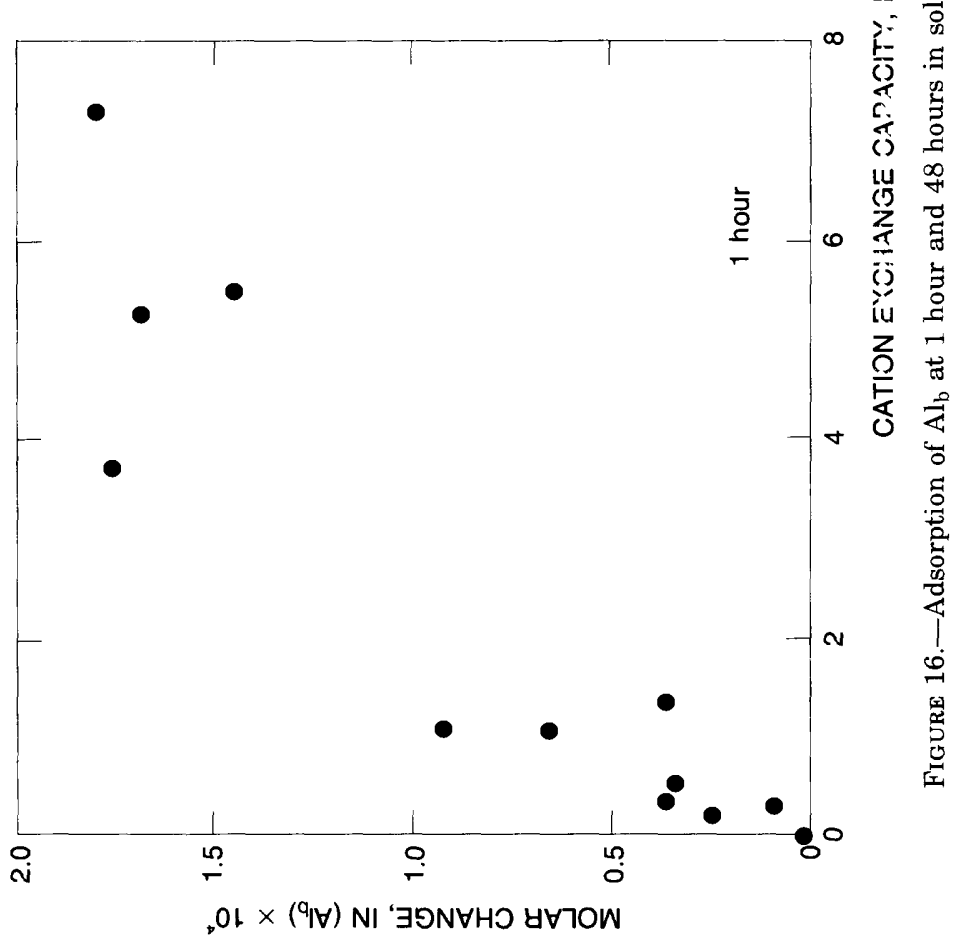




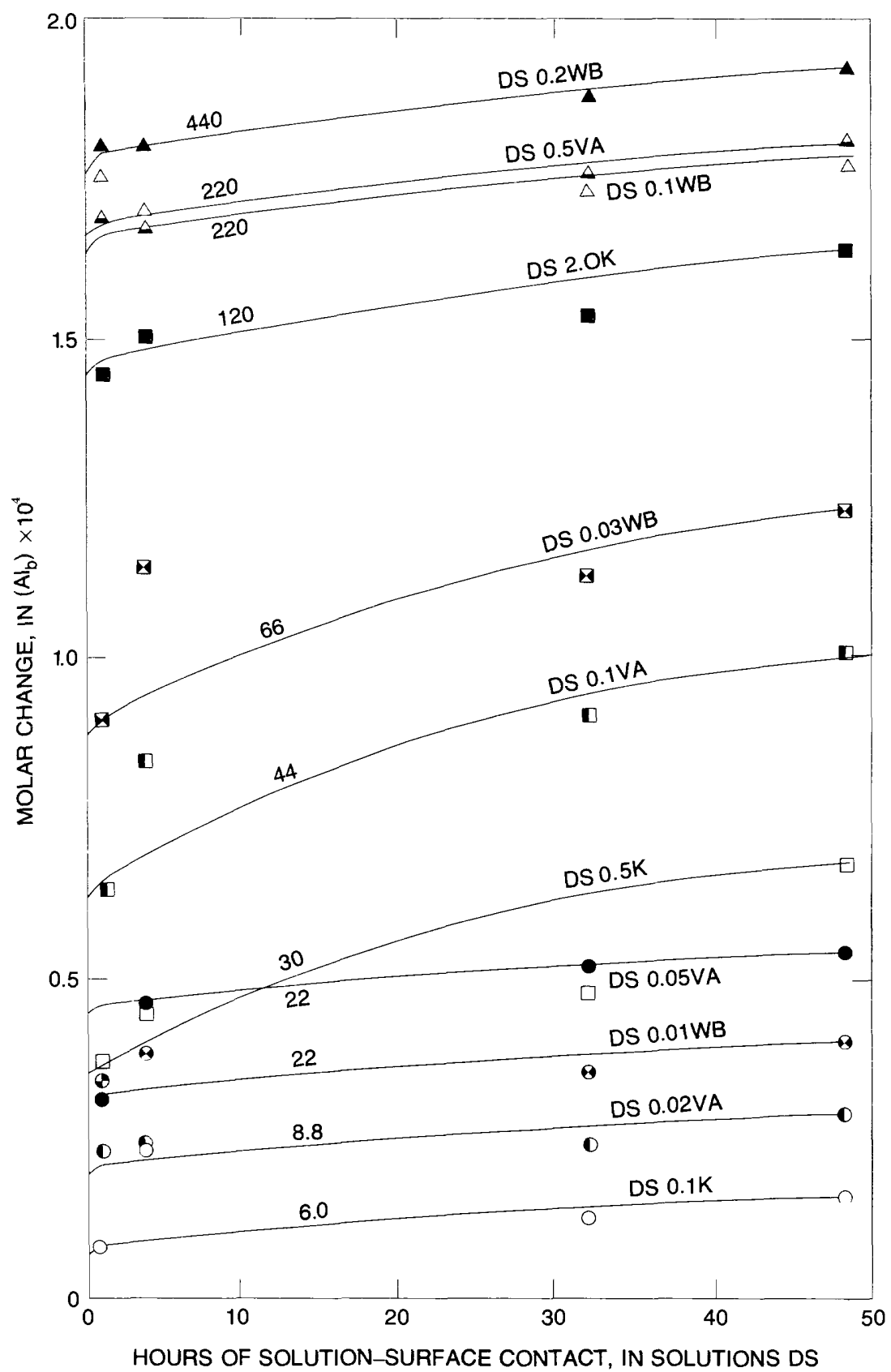

FIGURE 17.-Adsorption of $\mathrm{Al}_{\mathrm{b}}$ as a function of contact time for different amounts and types of mineral surfaces. Effective surface area in metres squared per litre is noted for each solution. 
Smith and Hem (1972) for estimating the size and charge of such species cannot be readily applied where the $\mathrm{pH}$ may not reflect correctly the increasing proportion of bridging hydroxide ions.

The apparent adsorption of $\mathrm{Al}_{\mathrm{b}}$ can, however, be studied by other procedures. Nearly all adsorption of $\mathrm{Al}_{\mathrm{a}}$ and most of that of $f \mathrm{l}_{\mathrm{b}}$ took place during the first hour of contact between the surfaces and solutions. Furthermore, for many of the solutions, aqueous concentrations of $\mathrm{Al}_{\mathrm{b}}$ also decreased between 1 and 48 hours. This effect is more pronounced for those solutions having the higher surface areas and is due to further adsorption by the surfaces or to an increased rate of polymerization of $\mathrm{Al}_{\mathrm{b}}$ catalyzed by the surfaces, or to a combination of both. In order to study this effect more closely, first-order rate constants were computed where possible, for the disappearance of $\mathrm{Al}_{\mathrm{b}}$ from the solutions during the period between 1 and 48 hours after preparation. Although there was a general tendency for a faster rate in solutions containing the greater amounts of surface, the ralationship was not very well defined. Rates of disappearance of $\mathrm{Al}_{b}$ from comparable aging-study solutions during longer aging periods generally are slower than rates for the 1-48-hour aged solutions ohserved here, but this comparison could only be made in a small number of solutions. Where a substantial amount of $\mathrm{Al}_{\mathrm{b}}$ was adsorbed before the first measurement, at 1 hour, the continuing loss of $\mathrm{Al}_{\mathrm{b}}$ may have been attributable to continued adsorption rather than to polymerization. We cannot definitely conclude from comparison of these rates with surface areas that the effect of surface area is to increase the pc'ymerization rate; however, if such an effect exists, it evidently is not great enough to be of major importance.

\section{EQUILIBRILM MODELS FOR ADSORPTION OF $\mathrm{Al}_{\mathrm{l}}$,}

The plots of adsorption of $\mathrm{Al}_{\mathrm{b}}$ as a function of surface ares clearly show that the adsorption per unit area of adsorbent approaches a limiting value as the surface area approaches zero. Kipling (1965) presented a form of the Langmuir adsorption isotherm that describes this type of behavior:

$$
\frac{a}{a_{\infty}}=\frac{b c}{1+b c},
$$

where $\alpha$ is the adsorption of solute per mass (or area) of adsorbent at aqueous solute concentration $c$. The limiting value of $\alpha$ at saturation is $a_{\infty}$, and $b$ is a constant. This equation may be rearranged as

$$
\frac{a}{c}=a_{\infty} b-b a \text {. }
$$

By plotting the quantity $a / c$ as a function of $a, a_{\infty}$ and $b$ can be 
determined. The relationship should define a straight line of intercept $a_{\infty} b$ and slope $-b$. The quantity $a$ represents the observed decrease in Al species concentration $(\Delta C)$ times sample volume $(T /)$ in litres divided by area of surface $(A)$. The quantity $c$ is the obser ed aluminum species concentration.

Data required for calculation of $a$ are given in table 8 for the 48 hour observations for $\mathrm{Al}_{\mathrm{b}}$. The results are given in table 11 . Figure 18 is a plot of the results.

TABLE 11.-Data used for development of Langmuir isotherm for adsorption of $\mathrm{Al}_{b}$

\begin{tabular}{|c|c|c|c|c|c|}
\hline Solution & $\underset{\left(\mathrm{m}^{2} / 1\right.}{A V}$ & $\underset{\text { (moles } / \text { litre) }}{C \times 10^{4}}$ & $\underset{(\text { moles/litre) }}{\Delta C \times 10^{+4}}$ & $\begin{array}{c}\frac{\Delta C \times 10^{4}}{A N} \\
\left(\mathrm{moles} / \mathrm{m}^{2}\right)\end{array}$ & $\begin{array}{c}\frac{\Delta C}{C A N N} \\
\left.\text { (litres } / \mathrm{m}^{2}\right)\end{array}$ \\
\hline DS NAS & 0.0 & 1.94 & 0.00 & & \\
\hline DS $0.01 \mathrm{WB}$ & 22 & 1.54 & .40 & 0.0182 & 0.0118 \\
\hline$\overline{\mathrm{DS}} 0.03 \mathrm{WB}$ & 66 & .70 & 1.24 & .0188 & .0268 \\
\hline DS $0.1 \mathrm{WB}$ & 220 & .14 & 1.80 & .0092 & .0584 \\
\hline DS $0.2 \mathrm{WB}$ & 440 & .01 & 1.93 & .0044 & 1.438 \\
\hline DS $0.02 \mathrm{VA}$ & 8.8 & 1.63 & .30 & .0341 & .0209 \\
\hline DS $0.05 \mathrm{VA}$ & 22 & 1.38 & .56 & .0254 & .0184 \\
\hline DS $0.1 \mathrm{VA}$ & 44 & .92 & 1.02 & .0232 & .0252 \\
\hline DS $0.5 \mathrm{VA}$ & 220 & .13 & 1.81 & .0082 & .0633 \\
\hline DS $0.1 \mathrm{~K}$ & 6.0 & 1.77 & .17 & .0280 & .0160 \\
\hline DS $0.5 \mathrm{~K}$ & 30 & 1.27 & .67 & .0224 & .0176 \\
\hline DS $2.0 \mathrm{~K}$ & 120 & .29 & 1.65 & .0137 & .0473 \\
\hline
\end{tabular}

${ }^{1}$ The measurement of aqueous $\mathrm{Al}_{\mathrm{b}}$ for solution $\mathrm{DS} 0.2 \mathrm{WB}$ gave a value at or below the detection limit $\left(10^{-6}\right.$ molar $)$ and, therefore, is a very highly uncertain $\frac{\Delta C}{C A N}$ value. Consequently, this point is not considered in determining slope
and intercept.

From inspection of figure 18, it is seen that the adsorption of $\mathrm{Al}_{b}$ could be explained by the Langmuir isotherm. The results are used to determine $a_{\infty}$ and b, which are found to be $3.7 \times 10^{-6} \mathrm{moles} / \mathrm{m}^{2}$ and $1.8 \times 10^{4}$ litres/mole, respectively. This says that at saturation, with an excess of $\mathrm{Al}_{b}$ remaining in solution, the surface would adsorb $3.7 \times 10^{-6}$ moles $\mathrm{Al}_{b}$ per square metre of surface area, or one atom in $\mathrm{Al}_{\mathrm{b}}$ per 45 square angstroms. Therefore, the maximum adsorption $a_{\infty}$ corresponds to less than a monolayer for our $\mathrm{Al}_{b}$ polymeric ions. The derived adsorption coefficient, $3.7 \times 10^{-6}$ moles $\mathrm{Al}_{\mathrm{b}} / \mathrm{m}^{2}$, compares reasonably well with the observed adsorption of $2.2 \times 10^{-6}$ moles $\mathrm{Al}_{\mathrm{b}} / \mathrm{m}^{2}$ in the test solutions where concentrations were as much as $2 \times 10^{-4}$ moles/litre.

From the foregoing discussion the following general conclusions are drawn:

1. $\mathrm{Al}_{\mathrm{b}}$ is rapidly removed from solution by adsorption when mineral surfaces are introduced into a system containing aqueous polynuclear aluminum hydroxide ions.

2. The adsorption process is related to surface area of the mineral available, and the relationship follows the pattern of the Langmuir isotherm. 


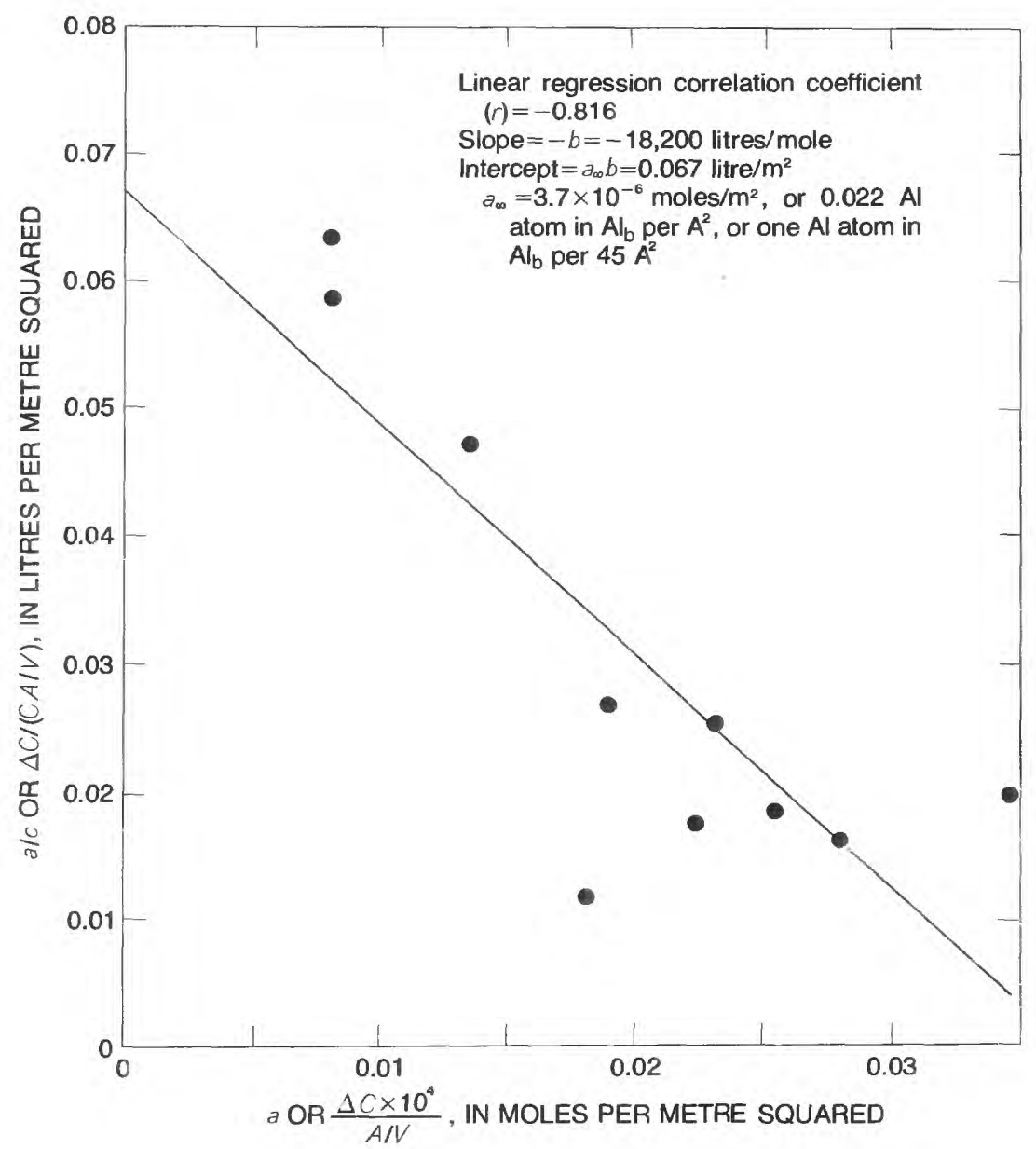

Figure 18.-Fit of $\mathrm{Al}_{\mathrm{b}}$ adsorption at 48 hours to Langmuir isotherm parameters.

3. Polymerization of $\mathrm{Al}_{\mathrm{b}}$ occurs in the solution at about the same rate whether a surface is present or not.

4. The initial take up of $\mathrm{Al}_{\mathrm{b}}$ by the surfaces does not appear to involve extensive polymerization because the differences in $\mathrm{pH}$ between blank solutions and those having various amounts of surfaces are small compared with the difference in $\mathrm{Al}_{b}$ content. Polymerization to the extent required to account for $\mathrm{Al}_{\mathrm{b}}$ conversion to $\mathrm{Al}_{\mathrm{c}}$ should produce enough $\mathrm{H}^{+}$to materially affect the $\mathrm{pH}$.

\section{BEHAVIOR OF Al}

As noted previously, aqueous $\mathrm{Al}_{c}$ cannot be directly determined from the available information. It can be calculated for solutions to which no mineral surface was added, and we know some $\mathrm{Al}_{c}$ was 
present at 48 hours in DS NAS (table 8). It seems reasonable that this material or its precursor also occurred in the other solutions before the mineral surfaces were added to them, but the development of recognizable crystallinity in this material is probably more rapid at the mineral surfaces. Because the change in solution $\mathrm{pH}$ is not large enough, it seems unlikely that very much of the $\mathrm{Al}_{\mathrm{b}}$ that disappeared was fully converted to $\mathrm{Al}_{c}$; however, the values for aqueous $\mathrm{Al}_{c}$ in table 8 were computed from the difference between determined $\mathrm{Al}_{\mathrm{a}}+\mathrm{Al}_{\mathrm{b}}$ and the determined total aqueous aluminum and thus should be reasonably valid. The quantity of adsorbed $\mathrm{Al}_{c}$ given for the $\mathrm{DS}$ solutions in table 8 represents the difference between aqueous $\mathrm{Al}_{c}$ in solution DS NAS and in solutions with mineral surfaces. In any event, the assumptions and calculations involved probably give the numbers for $\mathrm{Al}_{c}$ sorbed a rather high degree of uncertainty; efforts to correlate adsorbed $\mathrm{Al}_{\mathrm{c}}$ with surface area indicated only a rather tenuous relationship, and no quantitative treatment is presented here.

It is possible, however, by means of the electron microscope to detect the presence of $\mathrm{Al}_{\mathrm{c}}$ at the mineral surfaces as gibbsite crystals, which can be discerned in the electron micrographs of solids recovered from these solutions after approximately 100 days (figs. 19-21). The hex-

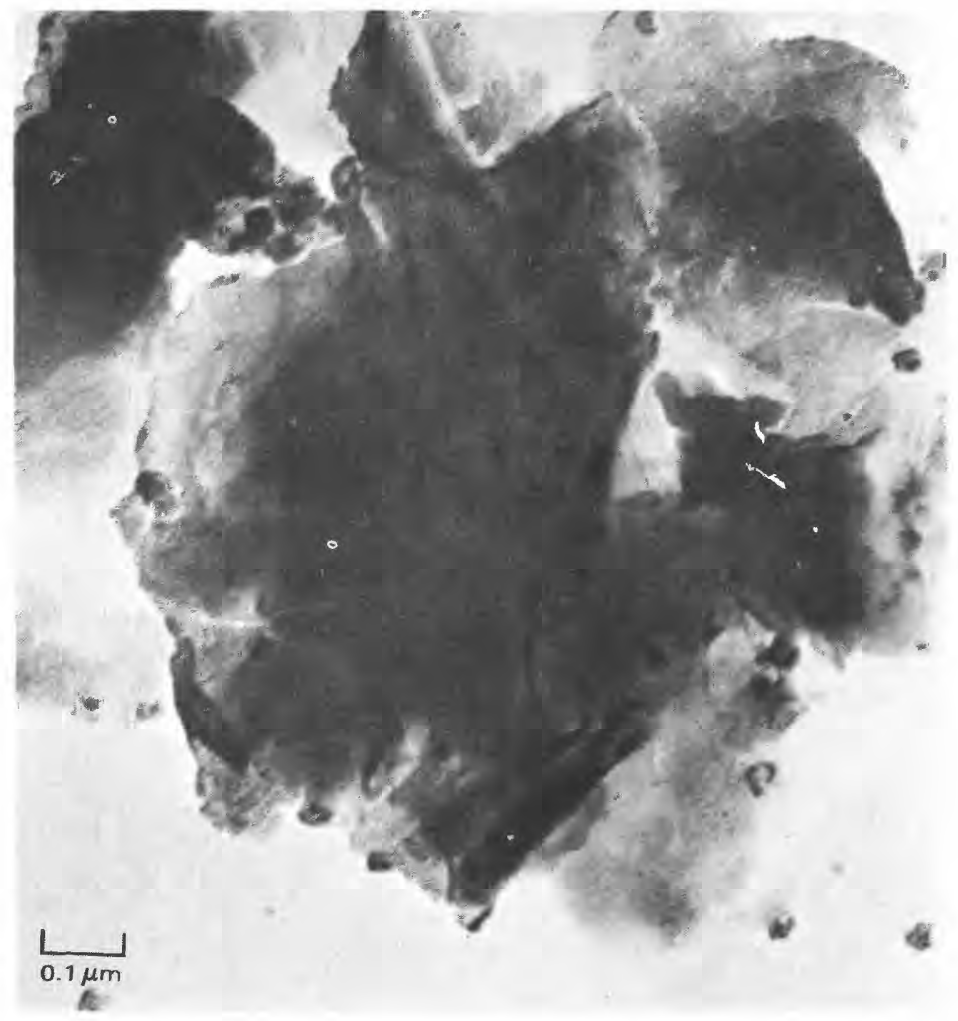

FiguRE 19.-Gibbsite crystallization centers on Wyoming bentonite after 100 days aging. 


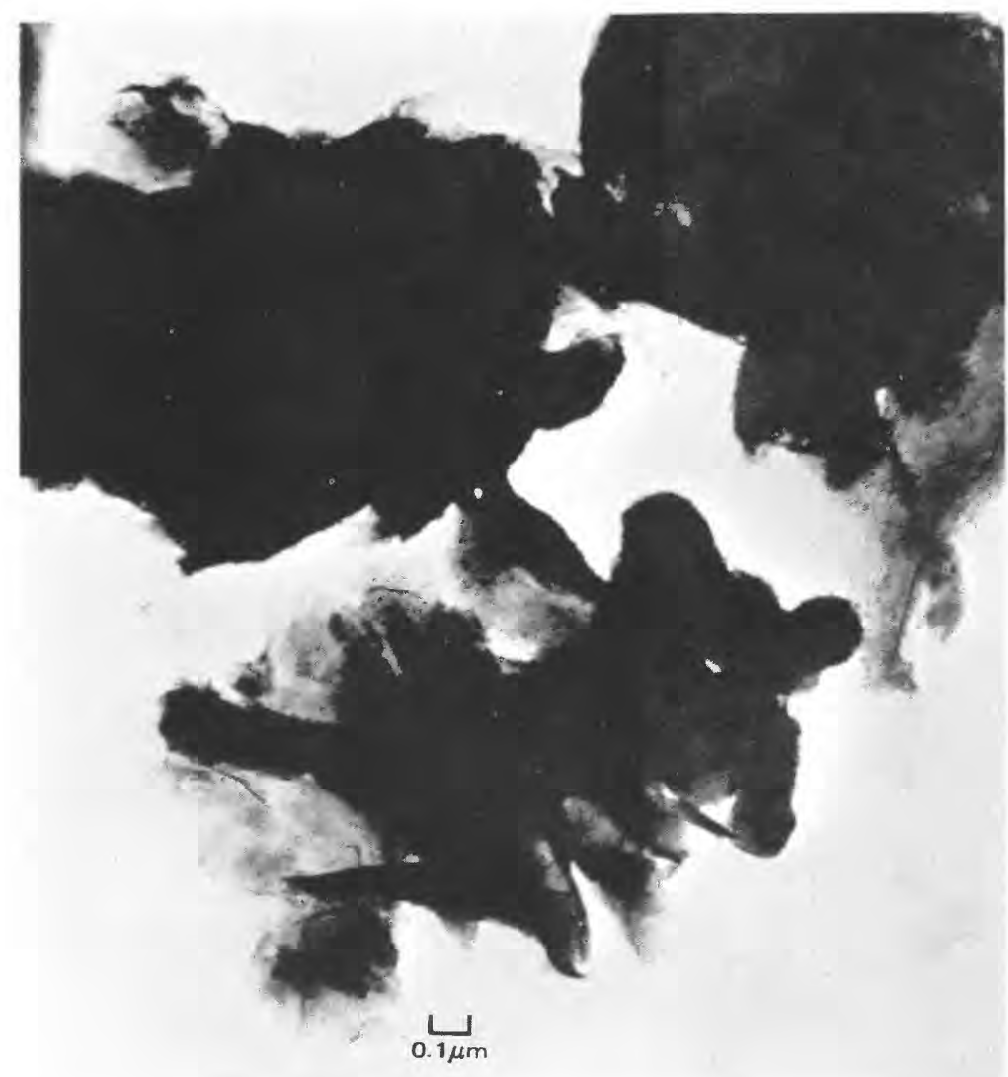

Figure 20.-Gibbsite crystals on surface of volcanic ash fragments after 100 days aging.

agonal plates adhere to the other mineral surfaces. Some of these crystals may have grown through polymerization of adsorbed $\mathrm{Al}_{\mathrm{b}}$, but direct sorption of the small crystals that had developed in the solution also has probably occurred. In solutions without surfaces that have been aged for a comparable length of time, one would be able to find similar gibbsite crystals that had developed independently.

Figure 22 shows a few gibbsite platelets on the surfaces of kaolinite removed from solution DS after 48 hours of aging. No crystals of gibbsite could be found in solutions without surfaces at this $\mathrm{pH}$ range in any of our previous work until after a longer aging period. Thus, the surfaces may facilitate the development of crystalline gibbsite, but as indicated by other evidence previously cited, the principal effect of the surfaces is that of adsorption.

\section{EFFECTS OF SURFACES ON APPROACH TO EQUILIBRIUM}

In previous work the solutions were assumed to have reached a state of equilibrium when the $\mathrm{Al}_{\mathrm{b}}$ had all disappeared, by conversion to $\mathrm{Al}_{\mathrm{C}^{\circ}}$. 


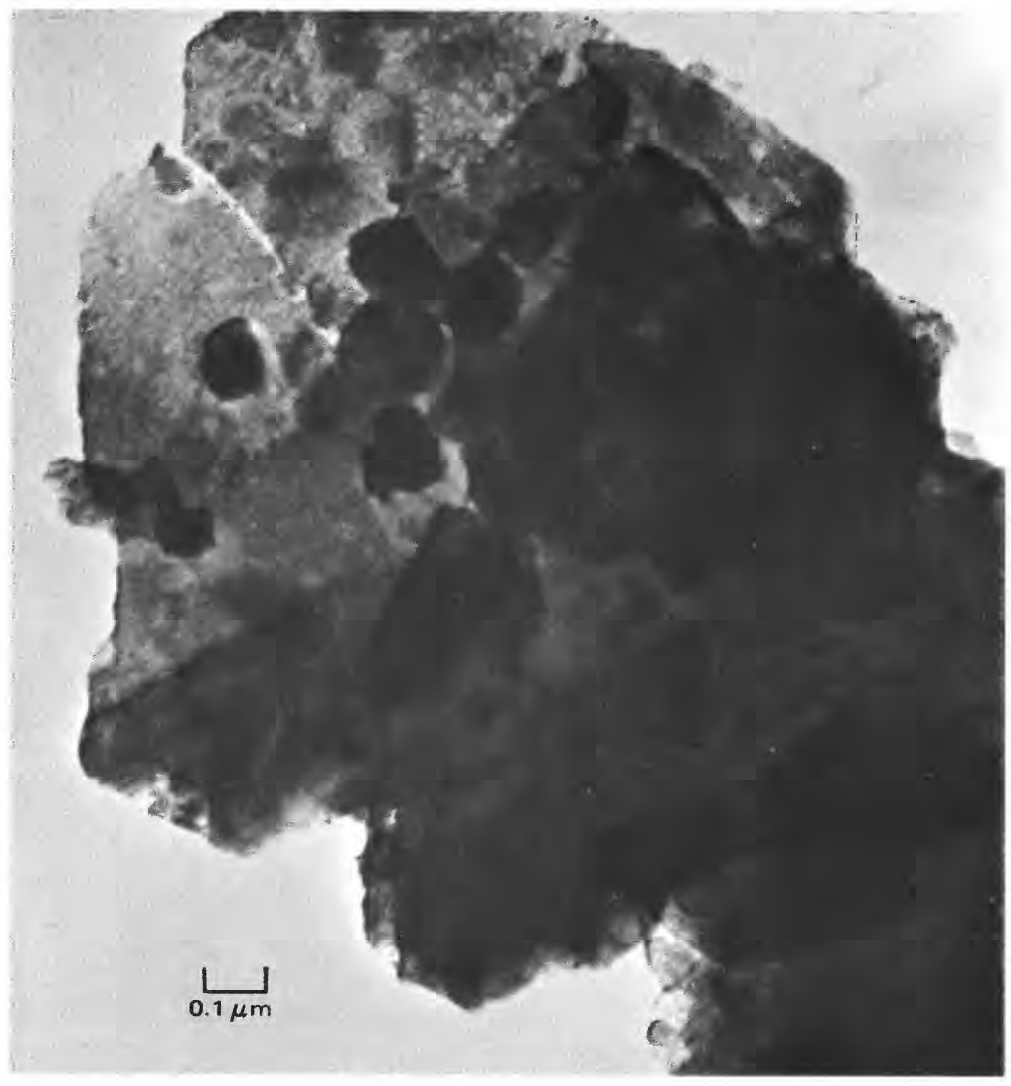

FIGURE 21.-Gibbsite crystals on surface of kaolinite after 100 days aging.

After that time, the $\mathrm{pH}$ remained constant. When the mineral surfaces were added, a substantial loss of dissolved aluminum commonly ensued, although it appeared generally not to be accompanied by a change in $\mathrm{pH}$ that was as large as would be expected were the process a polymerization of $\mathrm{Al}_{\mathrm{b}}$.

The approach to equilibrium in the absence of surfaces was traceable by the decreasing $\mathrm{pH}$. Actual changes in $\mathrm{Al}_{\mathrm{a}}$, although generally small, were observed in these solutions, probably because they had initially larger amounts of $\mathrm{Al}_{\mathrm{b}}$ than previously studied solutions where $\mathrm{Al}_{\mathrm{a}}$ remained nearly constant. In any event, the surfaces did help speed the attainment of equilibrium by adsorbing nonequilibrium species, where this was all that was needed to attain equilibrium. Where more extensive changes in solution composition were needed to attain equilibrium, the surfaces may not always promote such changes and could even inhibit them. However, the latter effect is probably rather rare. 


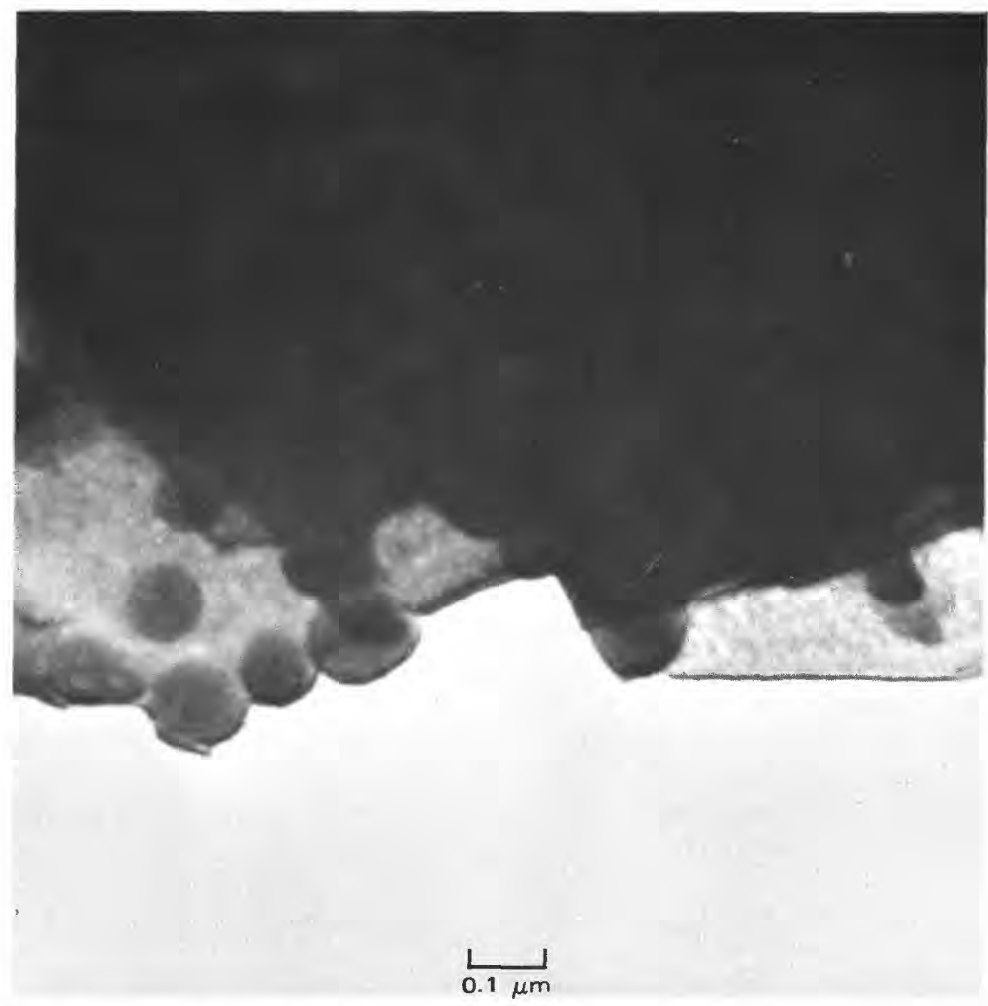

Figure 22.-Gibbsite platelets on surface of kaolinite after 48 hours aging.

\section{CONCLUSIONS REGARDING SORPTION AND POLYMERIZATION EFFECTS}

When a solution that is supersaturated with respect to gibbsite comes in contact with mineral surfaces having CEC, the experiments performed here suggest that a rapid decrease in dissolved aluminum species concentration occurs. The changes in concentration are most extensive during the first hour of reaction time and are closely correlated with effective surface area or CEC per unit volume of solution.

The weight of experimental evidence suggests that the aluminum species are removed by adsorption and that the adsorbed material does not undergo rapid polymerization to crystalline gibbsite. There may be some hydrolysis of monomeric dissolved aluminum species at the surface to increase the amount of hydroxide bound to aluminum, and microcrystals of gibbsite may be sorbed by the surfaces.

Surfaces used in these studies had some relatively subtle effects on distributions of dissolved species and rates of equilibration. However, after the initial adsorptive phase of the reactions was completed, the 
continued polymerization of the remaining $\mathrm{Al}_{\mathrm{b}}$ proceeded at the same rate and probably by the same mechanism as observed previously in solutions that had no added surface.

It should be noted that because surfaces were added after the initial mixing of $\mathrm{Al}^{+3}$ and $\mathrm{OH}^{-}$, the experiments do not permit evaluating the effect surfaces might have had on the initial formation of $\mathrm{Al}_{\mathrm{b}}$. Also, the $\mathrm{pH}$ range of the solutions was relatively narrow and was too low to include systems in which $\mathrm{Al}(\mathrm{OH})_{4}{ }^{-}$would have been a significant constituent. However, the results do indicate that the adsorption process can have significant effects on the behavior of aluminum in natural systems.

\section{REFERENCES CITED}

Bower, C. A., and Goertzen, V. O., 1959, Surface areas by an equilibrium ethylene gycol method: Soil Sci., v. 87, p. 289-292.

Brown, Eugene, Skougstad, M. W., and Fishman, M. J., 1970, Methods for collection and analysis of water samples for dissolved minerals and gases: U.S. Geol. Survey Techniques Water Resources Inv., book 5, chap. A1, 160 p.

Chapman, H. D., 1965, Cation exchange capacity, in Black, C. A., ed., Methods of soil analysis, part 2: Am. Soc. Agronomy ser. no. 9, p. 891-900.

Hem, J. D., 1964, Deposition and solution of manganese oxides: U.S. Geol. Survey Water-Supply Paper 1667-B, 42 p.

Hem, J. D., and Roberson, C. E., 1967, Form and stability of aluminum hydroxide complexes in dilute solution: U.S. Geol. Survey Water-Supply Paper 1827-A, 55 p.

Hem, J. D., Roberson, C. E., Lind, C. J., and Polzer, W. L., 1973, Chemical interactions of aluminum with aqueous silica at $25^{\circ} \mathrm{C}$ : U.S. Geol. Survey Water-Supply Paper 1827-E, $57 \mathrm{p}$.

Kipling, J. J., 1965, Adsorption from solutions of nonelectrolytes: London, Academic Press, 328 p.

Latimer, W. M., 1952, Oxidation potentials [2d ed.]: New York, Prentice-Hall, 392 p.

Lawrie, D. C., 1961, A rapid method for the determination of approximate surface areas of clays: Soil Sci., v. 92, p. 188-191.

Polzer, W. L., and Hem, J. D., 1965, The dissolution of kaolinite: Jour. Geophys. Research, v. 70, p. 6233-6240.

Robie, R. A., and Waldbaum, D. R., 1968, Thermodynamic properties of minerals and related substances at $298.15^{\circ} \mathrm{K}\left(25.0^{\circ} \mathrm{C}\right)$ and 1 atmosphere (1.013 bars) pressure and at higher temperatures: U.S. Geol. Survey Bull. 1259, 256 p.

Smith, R. W., and Hem, J. D., 1972, Effect of aging on aluminum hydroxide complexes in dilute aqueous solution: U.S. Geol. Survey Water-Supply Paper 1827-D, 51 p. 